\title{
Effect of Non-Equidiffusivity on Premixed Flame Propagation in Obstructed Channels
}

Gbolahan Idowu

Follow this and additional works at: https://researchrepository.wvu.edu/etd

\section{Recommended Citation}

Idowu, Gbolahan, "Effect of Non-Equidiffusivity on Premixed Flame Propagation in Obstructed Channels" (2017). Graduate Theses, Dissertations, and Problem Reports. 5854.

https://researchrepository.wvu.edu/etd/5854

This Thesis is protected by copyright and/or related rights. It has been brought to you by the The Research Repository @ WVU with permission from the rights-holder(s). You are free to use this Thesis in any way that is permitted by the copyright and related rights legislation that applies to your use. For other uses you must obtain permission from the rights-holder(s) directly, unless additional rights are indicated by a Creative Commons license in the record and/ or on the work itself. This Thesis has been accepted for inclusion in WVU Graduate Theses, Dissertations, and Problem Reports collection by an authorized administrator of The Research Repository @ WVU. For more information, please contact researchrepository@mail.wvu.edu. 


\title{
Effect of Non-Equidiffusivity on Premixed Flame Propagation in Obstructed Channels
}

\author{
Gbolahan Idowu
}

\begin{abstract}
Thesis submitted to the
Benjamin M. Statler College of Engineering and Mineral Resources at West Virginia University
\end{abstract}

in partial fulfillment of the requirements for the degree of

\author{
Master of Science \\ in Mechanical Engineering \\ V'yacheslav Akkerman, Ph.D., Chair \\ Hailin Li, Ph.D. \\ Arvind Thiruvengadam, Ph.D.
}

Department of Mechanical and Aerospace Engineering

Morgantown, West Virginia

2017

Keywords: premixed combustion, non-equidiffusive, flame propagation, flame acceleration, thermal expansion, flame front, deflagration-to-detonation transition, obstructed channels, Lewis number, Reynolds number, blockage ratio 


\section{Abstract \\ Effect of Non-Equidiffusivity on Premixed Flame Propagation in Obstructed Channels}

\section{Gbolahan Idowu}

Continuous fire safety hazards stimulate in-depth learning and understanding of what causes an initially slow premixed flame (deflagration) front to accelerate and eventually detonate. Such flame acceleration (FA), presumably followed by a deflagration-to-detonation transition (DDT), are intriguing phenomena that have both fundamental interests and practical relevance. On one hand, it is the desire to prevent FA and DDT to avoid or, at least, mitigate unwanted explosions or fires. On the other hand, FA and DDT can be utilized, constructively, in the novel energyefficient technologies such as micro-combustors, rotation-detonation engines or pulse-detonation engines. A flame accelerates in tubes or channels, with acceleration being most intensive in obstructed pipes. The latter fact has been known for a while, but this acceleration was typically devoted to turbulence or shocks. In contrast, the Bychkov scenario of FA in channels, equipped with a tooth-brush-like array of tightly-packed obstacles, is shockless and conceptually laminar, with turbulence playing only a supplementary role. In spite of the laminar nature, this FA is extremely strong and leads to DDT.

The geometry of the Bychkov mechanism is the following: one end of an obstructed channel is closed, while the other end is open or vented; a flame embryo is ignited at the closed end, and then it accelerates towards the open one. This mechanism has been identified and quantified analytically and substantiated by the comprehensive computational simulations. However, the Bychkov theory and modeling employed various simplifications, including that of equidiffusive combustion, which means that the Lewis number, Le (the thermal-to-mass diffusivities ratio) is unity. While the latter is a conventional approach in combustion science, flames are usually nonequidiffusive in the practical reality, with $L e$ belonging to the key parameters controlling the flame dynamics and morphology. Consequently, there has been a critical need to scrutinize the impact of the Lewis number in obstructed channels, which is addressed in the present thesis.

Specifically, acceleration of non-equidiffusive flames in obstructed channels is investigated by means of computational simulations of the reacting flow equations with fully-compressible hydrodynamics and Arrhenius chemical kinetics. A detailed parametric study is performed for the Lewis numbers in the range $0.2 \leq L e \leq 2.0$, blockage ratios (BR) being $1 / 3 \sim 2 / 3$, the spacing between the obstacles $\Delta \mathrm{z} / \mathrm{R}=1 / 4 \sim 1 / 2$, and the channel width $48 \leq D / L_{f} \leq 96$, where $L_{f}$ is the thermal flame thickness. It is shown that $L e>1$ flames accelerate slower than equidiffusive ones, due to flame thickening. In contrast, $L e<1$ flames acquire stronger distortion, associated with the diffusional-thermal combustion instability, and thereby accelerate much faster than at $L e=1$. 


\section{Acknowledgement}

I would like to use this opportunity to thank all the key stakeholders who have been instrumental to the success of my thesis completion. Indeed, there were challenging times, but I am glad to have had the support and guidance of these individuals.

Firstly, I would like to thank Dr. V'yacheslav Akkerman, my research advisor throughout my Master's program. I could not have asked for a more patient, kind and inspiring role model. $\mathrm{He}$ willingly took me under his wing once I expressed my interest in his research group, even though his team was already full at that time. He is truly a one of a kind professor and I will be forever grateful to him for his never wavering encouragement.

Secondly, I would like to express my deepest gratitude to all members of my committee. Even with knowing me sparingly, Dr. Thiruvengadam and Dr. Li agreed to join my committee with much enthusiasm. I am truly grateful to both of them for making time for me out of their busy schedules.

Thirdly, I can't imagine how life would have been as a research student, if I was not surrounded by such a talented group of colleagues like I had in my research group. I want to say a special thanks to Sinan, Afeez, Furkan, Swathi and Rawan for always being there for me whenever I needed assistance. I also want to thank our undergraduate student Amanda for all her hard work and dedication in helping me progress efficiently in completing my research work.

Lastly, I would like to extend my heartfelt gratitude to my girlfriend, family in Nigeria and U.S., who all have remained ever supportive throughout my quest to pursue a Master's degree. I am truly blessed to call them family and I am determined to continually make them proud. 


\section{Dedication}

I would like to dedicate this work to my girlfriend, parents and siblings who have always remained a tremendous support system to me all throughout my academics. Without their love and encouragement, I would not be the individual I am today. 


\section{Table of Contents}

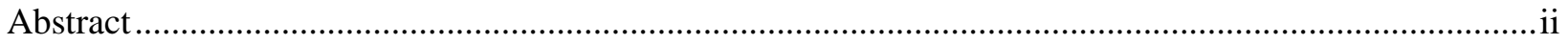

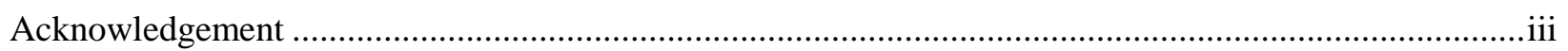

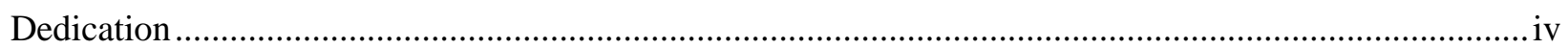

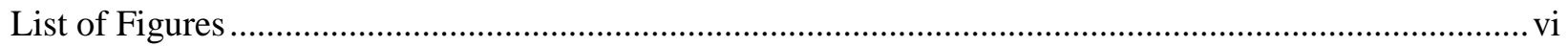

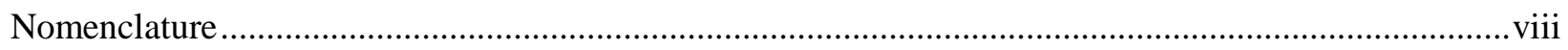

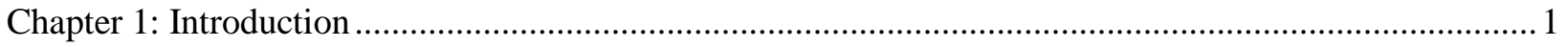

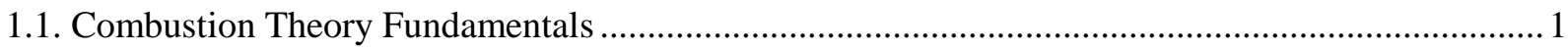

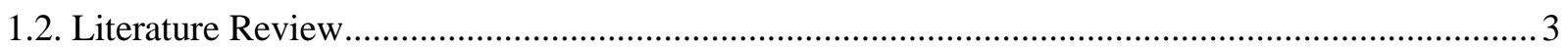

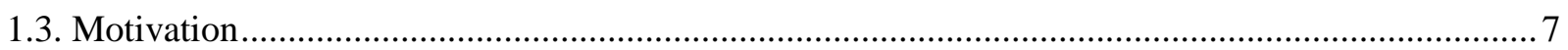

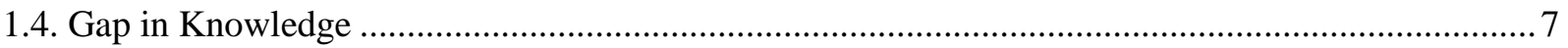

Chapter 2: Flame Acceleration in Obstructed Channels .....................................................................

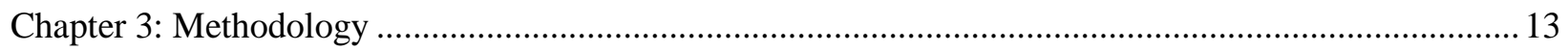

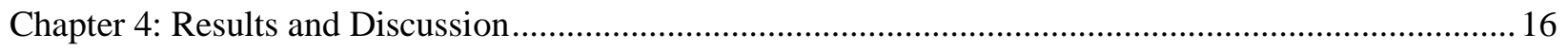

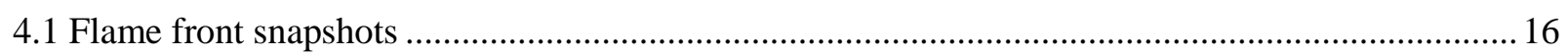

4.2. Effect of Lewis number on flame acceleration ................................................................... 17

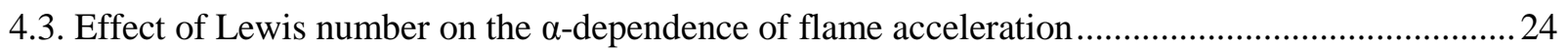

4.4. The effect of Lewis number on the Re-dependence of flame acceleration ................................... 32

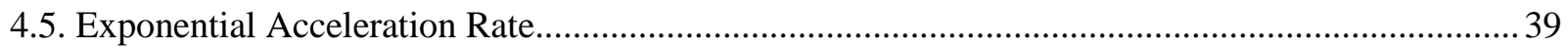

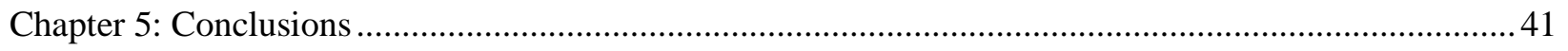

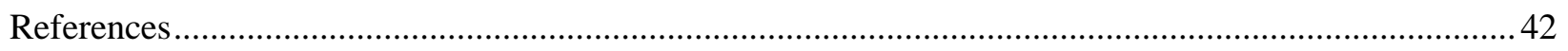




\section{List of Figures}

Figure 1.1- Schematic of the structure of a premixed flame front [1]

Figure 1.2- Schematic of the temperature and density profile inside a premixed flame front [1]

Figure 1.3- DDT applications [1]

Figure 1.4- Finger flame acceleration mechanism [3]

Figure 1.5- (a) Wall friction mechanism schematic and (b) computational simulation [1]

Figure 2.6- Laminar mechanism of flame acceleration in a semi-open channel filled with obstacles [8]

Figure 2.7- A color snapshot from a numerical simulation of FA in a channel with thinly packed obstacles

Figure 4.8- Temperature snapshot [from $300 \mathrm{~K}$ in the fuel mixture (blue) to $2400 \mathrm{~K}$ in the burnt matter] at the same time instant i.e. scaled time, $\tau=0.075$ for $R e=24$ and various Le and $\alpha$

Figure 4.9- Scaled flame tip velocity as a function of scaled time for fixed $R e=24$

Figure 4.10- Scaled flame tip velocity as a function of scaled time for fixed Re=36

Figure 4.11- Scaled flame tip velocity as a function of scaled time for fixed Re=48

Figure 4.12- Temperature profiles for flames with $R e=48, \alpha=2 / 3$ and Le=0.2 for various scaled times $\tau=0.043(a) 0.063(b) 0.078(c)$

Figure 4.13- Temperature profiles for flames with $R e=48, \alpha=2 / 3$ and Le $=1.0$ for various scaled times $\tau=0.043(a) 0.063(b) 0.078(c)$

Figure 4.14- Temperature profiles for flames with $R e=48, \alpha=2 / 3$ and Le=2.0 for various scaled times $\tau=0.043($ a $) 0.063$ (b) 0.078 (c)

Figure 4.15- Scaled flame tip velocity as a function of scaled time for fixed Re=24

Figure 4.16- Scaled flame tip velocity as a function of scaled time for fixed Re=36

Figure 4.17- Scaled flame tip velocity as a function of scaled time for fixed Re=48

Figure 4.18- Temperature profile of flames with $R e=36, L e=1.0$ and $\alpha=1 / 3$ for various scaled times $\tau=0.046(a), 0.066(b), 0.079(c)$

Figure 4.19- Temperature profile of flames with $R e=36, L e=1.0$ and $\alpha=1 / 2$ for various scaled times $\tau=0.046(a), 0.066(b), 0.079(c)$ 
Figure 4.20- Temperature profile of flames with $R e=36, L e=1.0$ and $\alpha=2 / 3$ for various scaled times $\tau=0.046(a), 0.066(b), 0.079(c)$

Figure 4.21-Scaled flame tip velocity as a function of scaled time for fixed $\alpha=1 / 3$

Figure 4.22- Scaled flame tip velocity as a function of scaled time for fixed $\alpha=1 / 2$

Figure 4.23-Scaled flame tip velocity as a function of scaled time for fixed $\alpha=2 / 3$

Figure 4.24- Temperature profile of flame with $L e=0.2, \alpha=1 / 3$ and $R e=24$, for various scaled times $\tau=0.046($ a) 0.062 (b) 0.078 (c)

Figure 4.25- Temperature profile of flame with Le=0.2, $\alpha=1 / 3$ and $R e=36$, for various scaled times $\tau=0.046($ a $) 0.062$ (b) $0.078(c)$

Figure 4.26- Temperature profile of flame with $L e=0.2, \alpha=1 / 3$ and $R e=48$, for various scaled times $\tau=0.046($ a $) 0.062$ (b) $0.078(c)$

Figure 4.27- The exponential acceleration rate $v$ s the Lewis number for fixed $R e=24,36,48$

Figure 5.28-Scaled flame tip position as a function of scaled time, for various $\alpha=1 / 3,1 / 2,2 / 3$ and $L e=0.2,1.0,2.0$ 


\section{Nomenclature}

Latin

\begin{tabular}{|c|c|}
\hline$A_{w}$ & Scaled flame surface area \\
\hline$C_{P}$ & Heat capacity at constant pressure \\
\hline$C_{V}$ & Heat capacity at constant temperature \\
\hline$C I$ & Compression ignition \\
\hline$D_{f}$ & Length of a 2D flame front \\
\hline$D_{t h}$ & Thermal diffusion coefficient \\
\hline$D L$ & Darrius-Landau \\
\hline$D T$ & Diffusional-thermal \\
\hline$D D T$ & Deflagration-to-detonation transition \\
\hline$E_{a}$ & Activation energy \\
\hline$f$ & Flame shape function \\
\hline$F A$ & Flame acceleration \\
\hline$H$ & Enthalpy \\
\hline$L_{f}$ & Flame thickness \\
\hline Le & Lewis number \\
\hline$m$ & Species molecular weight \\
\hline$M a$ & Mach number \\
\hline$M k$ & Markstein number \\
\hline$P$ & Pressure \\
\hline$P_{f}$ & Flame pressure \\
\hline$P D E$ & Pulse detonation engine \\
\hline $\operatorname{Pr}$ & Prandtl number \\
\hline$Q$ & Specific energy \\
\hline$R_{u}$ & Universal gas constant \\
\hline$R e$ & Flame Reynolds number \\
\hline Sc & Schmidt number \\
\hline$T$ & Temperature \\
\hline$T_{b}$ & Temperature of burnt matter \\
\hline$T_{f}$ & Flame temperature \\
\hline$U_{f}$ or $S_{L}$ & Planar flame speed \\
\hline
\end{tabular}




$\begin{array}{ll}U_{w} & \text { Flame speed near the wall } \\ Y & \text { Mass fraction of the fuel } \\ Z e & \text { Zeldovich number }\end{array}$

Greek

$\begin{array}{ll}\gamma_{i j} & \text { Stress tensor } \\ q_{i} & \text { Energy diffusion vector } \\ \alpha & \text { Blockage ratio } \\ \varepsilon & \text { Specific internal energy } \\ \Theta & \text { Thermal expansion coefficient } \\ \zeta & \text { Dynamic viscosity } \\ v & \text { Kinematic viscosity } \\ \rho & \text { Density } \\ \sigma & \text { Exponential acceleration rate } \\ \tau & \text { Scaled time } \\ \tau_{R} & \text { Time dimension constant }\end{array}$




\section{Chapter 1: Introduction}

\subsection{Combustion Theory Fundamentals}

Combustion is a process of mass and energy conversion through which chemical bond energy is transformed into thermal energy. A fuel source and oxidizer are essential for this reaction to occur. With several other energy sources available, it is remarkable that combustibles still play a key role in human operations. Fossil fuels provide a significant percentage (up to 85\%) of the US energy needs (manufacturing, heating, cooking etc.) [1]. It also plays a vital role in meeting societal transportation and aviation demands.

There exist two major regimes of combustion, namely, premixed and non-premixed (diffusion) combustion. Premixed combustion: here, the fuel is already mixed with the oxidizer before the reaction starts. Spark-ignition (SI) engines are the most common application of premixed combustion. Diffusion combustion: here, the fuel and oxidizer are initially separated and do not mix until the combustion process starts; Diesel engines, also known as compression ignition (CI) engines, operate in diffusion combustion mode during NOx formation (although soot formation is associated with premixed combustion mode).

Premixed combustion can be further classified into two regimes, namely: deflagration and detonation [1]. Deflagration, also known as flame, is a regime in which the reaction propagates due to thermal conduction. It is characterized by slow subsonic flow, with speeds $\sim 1 \mathrm{~m} / \mathrm{s}$, i.e. the Mach number $M \ll 1$. In the case of detonation, the reaction propagates due to shock waves. Consequently, it goes with supersonic flow speeds, i.e. $>300 \mathrm{~m} / \mathrm{s}$ and $M>1$. 


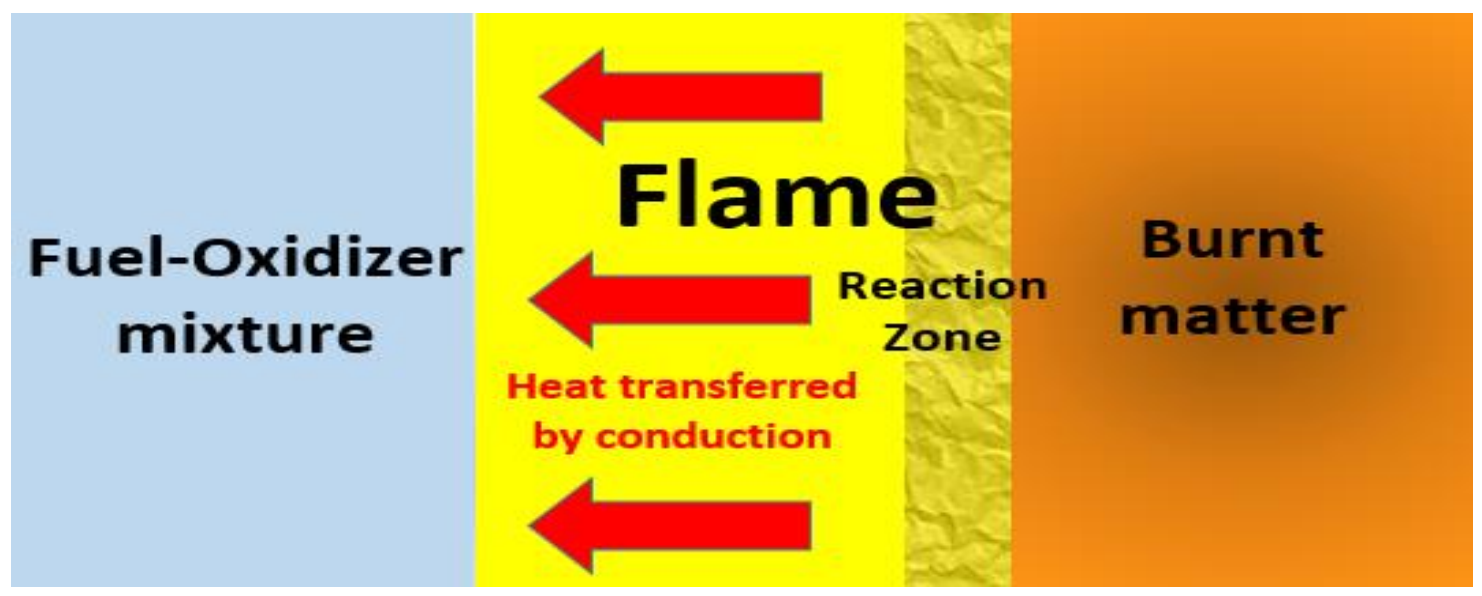

Figure 1.1-Schematic of the structure of a premixed flame front [1]

The primary focus of this thesis is devoted to premixed flames, and Figure 1.1.1 shows the distinctive regions for such a combustion wave. When a flame is ignited, it goes away from the source and travels to consume more fuel-oxidizer mixture. The heat, released in the reaction, is transferred to the cooler mixture by heat conduction. The reaction occurs in the reaction zone and the by-products are retained behind the flame front as burnt matter. Figure 1.2 below shows the temperature and density profiles inside a flame front. As the flame moves from the right to the left towards the fuel-air mixture, the temperature ahead of the flame diminishes while the density increases due to thermal expansion. The shaded red region indicates a so-called "active reaction zone", where the temperature is close to the burnt matter temperature and thereby where the reaction rate is the highest and, therefore, where the reaction actually occurs.

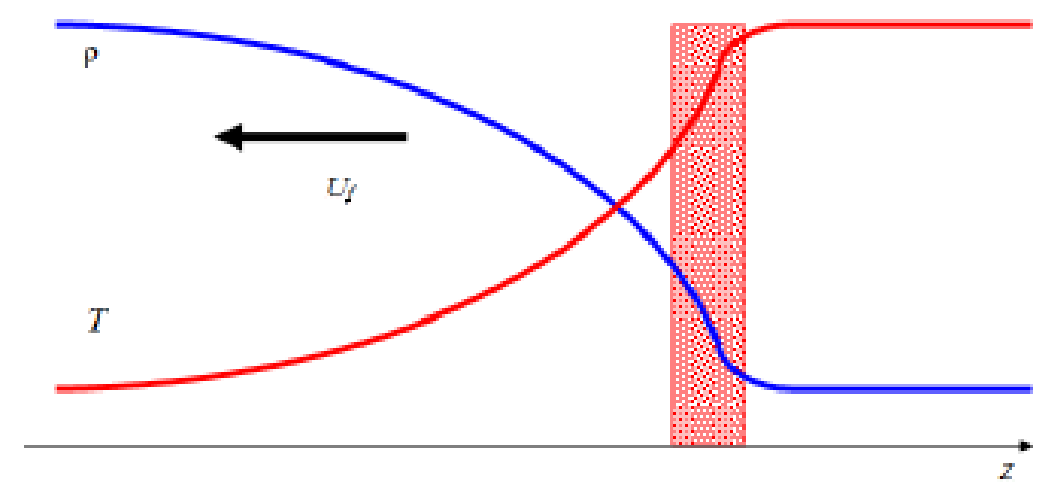

Figure 1.2- Schematic of the temperature and density profile inside a premixed flame front [1]

It is noted that a flame may spontaneously accelerate and subsequently trigger detonation. This phenomenon is known as the deflagration-to-detonation transition (DDT). It is encountered in nature and technology and may constitute a conceptual hazard. Various DDT applications are 
illustrated in Figure 1.3. Accidental explosions in power plants and rockets as well as disasters in coalmines are just several examples of severe hazards associated with uncontrolled DDT. However, DDT could also be constructively utilized because the energy released from this process is valuable if effectively handled. This has piqued the interest of several remarkable designs such as pulse-detonation engine (PDE) technologies.
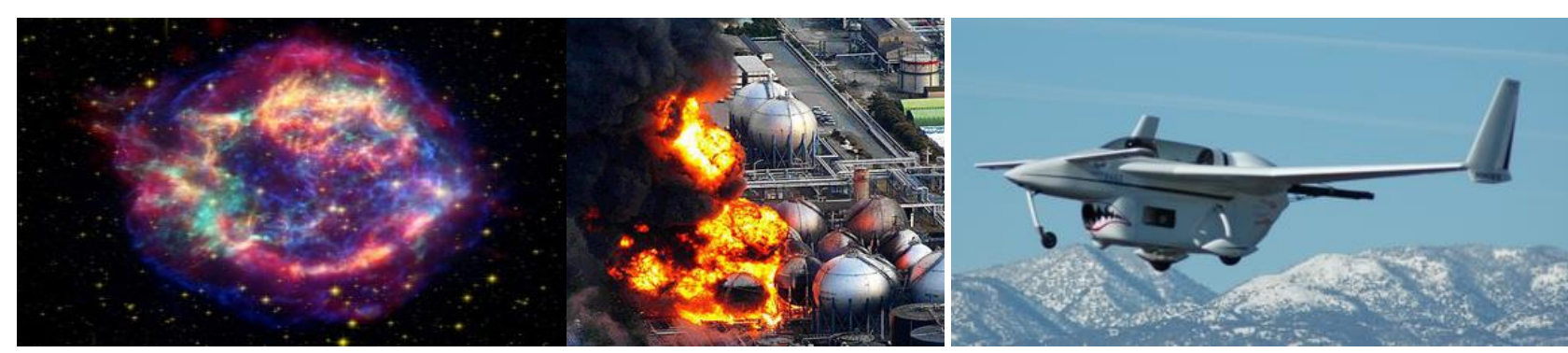

Figure 1.3-DDT applications: (a) Thermonuclear supernovae (b) Plant disaster (c) Pulse-detonation engine [1]

\subsection{Literature Review}

Studies on flame acceleration (FA) and DDT have served among the primary concerns of the fire safety industry. Over the years, several explosion catastrophes have been attributed to DDT, paving the way for more aggressive research in this area. More recently, in April 2010, a coal mine disaster claimed the lives of 29 miners, due to a coal dust explosion (Upper Big Branch in WV). The primary cause was the ignition of methane, the major constituent in coalmines, which is a gas that can easily trigger explosions [2]. Indeed, accidental explosions are difficult to handle, however, the energy released from such explosions can be harnessed for specific engines, i.e. PDEs. Prior research has been conducted to analyze the factors that cause flames to accelerate in a smooth channel. The first fundamental mode of FA in channels is the so-called finger flame acceleration. The thermal and mechanistic boundary conditions employed in this model are slip and adiabatic walls [3]. Upon ignition, the flame acquires a hemispherical shape. Then it becomes non-uniform. It accelerates mainly axially, leading to a finger shape. The drawback to this model is that it is limited in time and acceleration stops when the flame skirt touches the wall of the channel. Figure 1.4 shows a schematic and temperature snapshots of this acceleration mechanism. The growth of the flame surface area can be seen in the snapshots I-IV. The flame still takes on its finger flame shape in V, when the flame skirt touches the wall, but thereafter acceleration is terminated. 
The Shelkin mechanism expanded on this prior model of acceleration by introducing the effect of wall friction i.e. non-slip boundary conditions. This leads to a more sustained mode of FA that is not limited in time and can invariably lead to detonation [3]. Figure 1.5 below shows temperature snapshots of the Shelkin mechanism of FA. It is evident how the flame front accelerates due to wall friction as it does so almost during the entire propagation through the channel.

(a)
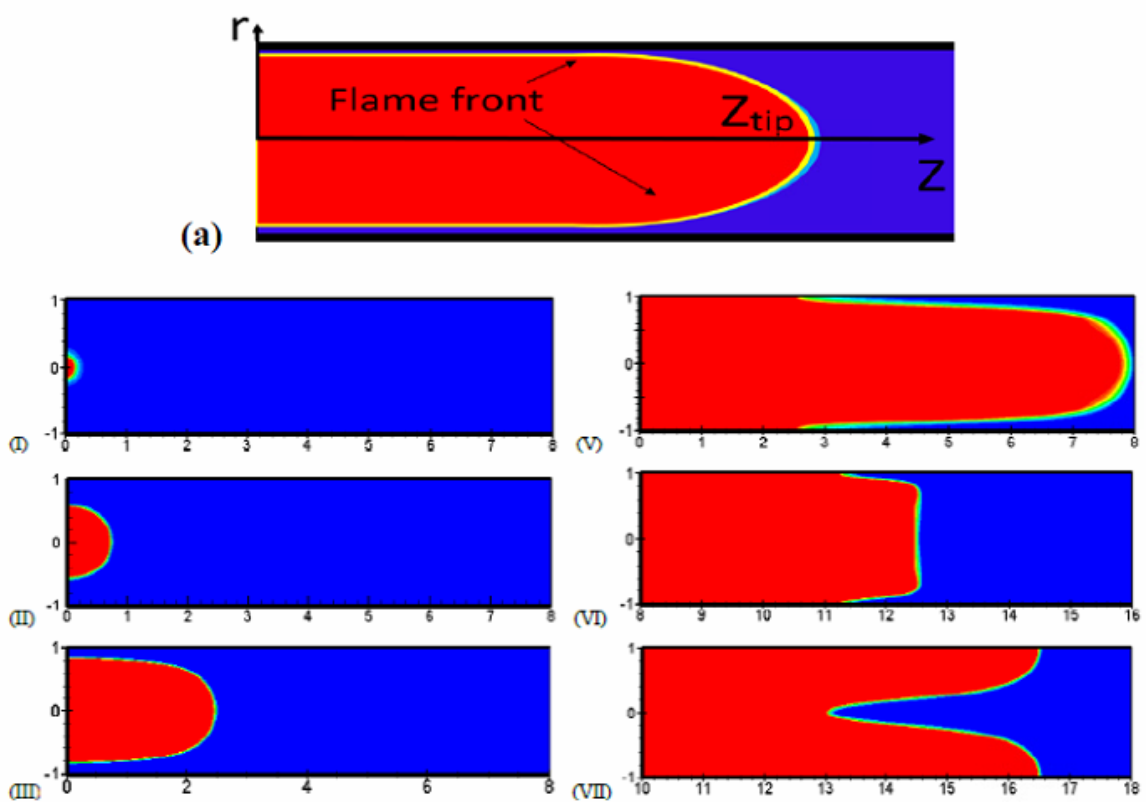

(b)
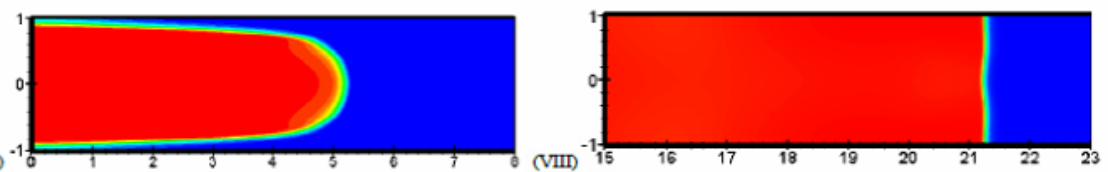

Figure 1.4 - Finger flame acceleration mechanism (a) illustration (b) computational snapshots[3] 


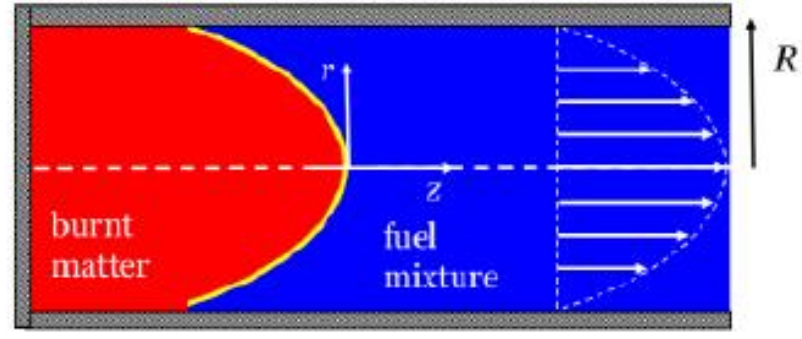

(a) (b)

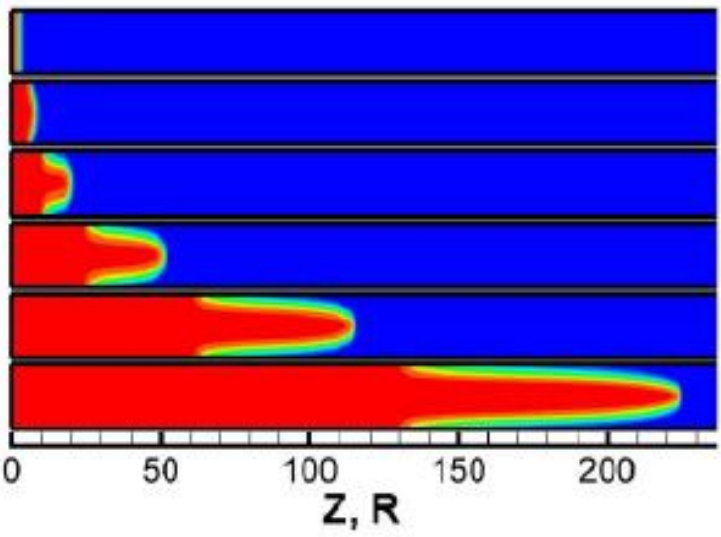

Figure 1.5 -(a) Wall friction mechanism schematic and (b) computational simulation [1]

These pioneering research results led to other substantial findings in the quest to fully identify the factors that influence FA in channels and tubes. Among them include, the work of Silvestrini et al [4] which aimed at comparing FA in a smooth tube to FA in a tube filled with obstacles and determine the run-up distance: the length of a channel needed for a flame to effectively transform to a detonation wave. It was identified that factors such as the nature and composition of the fuel mixture as well as the channel geometry, greatly impacted the mode of acceleration. It was believed that the presence of obstacles created a turbulent disturbance of the flame, increasing the burning rate thereby leading to rapid acceleration [4].

Ciccarelli et al [5] went further by investigating the effect of obstacle size and spacing on the initial stage of FA in a rough tube. It was established that higher blockage ratios improved the occurrence of detonation, by causing an increase in the flame surface area in the early stages of acceleration. In contrast, later stages of acceleration are influenced by combustion instabilities [5]. Johansen et al [6] also employed a similar approach analyzing the impact of the obstacles' blockage area and the conclusions of Ref. [6] agreed with the previous works.

Although several studies of FA in channels with obstacles attribute this acceleration to turbulence [4,5], Bychkov et al [7] have identified a laminar, shockless mechanism of ultrafast FA in semi-open obstructed channels (one end of a channel is open while the other is closed such that the flame is ignited at the closed end and moves to the open one). This acceleration is driven by a jet-flow generated when a flame propagates through a "tooth-brush"-like array of obstacles. The latter constitutes the focus of the present thesis and will be explained in detail in Chapter 2.

While semi-open channels have proven to be a primary focus for several studies of ultrafast FA [7-9], channels with both open ends have also been studied. In particular, the experimental 
measurement and computational simulations by Yanez et al [10] attributed sudden acceleration of flames in open channels to hydraulic resistance. It was identified that channels with both ends open exhibit a phenomenon quite different from the classical DDT scenarios. Namely, a flame does not accelerate right after the ignition; in contrast, it experiences a delay prior to sudden acceleration, presumably, followed by DDT. The computational study of Middha \& Hansen [11] obtained the same delay prior to sudden acceleration. It was suggested that the interaction of the expanded gas with the obstructed walls of the channel obstacle creates a turbulent feedback, which promotes the flame speed rapidly [11]. A similar result has been recently identified in the analytical study of FA in a vented obstructed channel by Bychkov et al [12]. The acceleration characteristics in channels with both ends open were found to be considerably weaker as compared to that in semi-open channels. The reason for this reduced acceleration is a distribution of the flame-generated gas volume between the flows directed towards both ends of the pipe. Viscous forces were also attributed to moderation of FA before the onset of strong acceleration.

Heidari et al [13] went ahead to study computationally, the DDT phenomena for a hydrogen-air mixture. The latter was chosen because, although being generally very explosive, hydrogen is nevertheless proven to be one of the safest gases, considering its low ignition energy. It was noticed that hot spots were created in the unburnt gases due to pressure oscillations inside the flame front, which invariably accelerate the flame and could trigger detonation. Hence, the occurrence of DDT is due to preheating of the unburnt gases ahead of the flame and the subsequent interaction with the flame causes the releases of a strong shock wave [13]. Similarly, Kessler et al [2] undertook a numerical approach to study FA in channels but for a different premixture: a methane-air mixture. Considering that methane is typically found in a mining atmosphere, this research provided great insight in the protection of personnel, equipment and operations from mine explosions. The followed stages of FA have been distinguished:

- Flame stretching and folding;

- Flame-front wrinkling (believed to be caused by turbulence) and fluid dynamic instabilities;

- Increase in flame surface area caused by the shock-flame interaction.

It was identified that when the velocity of the burnt matter approaches the speed of sound, the shock-flame interactions begin impacting the DDT scenario. The appearance of hot spots behind a shock wave were also attributed to detonation triggering as postulated by Heidari et al [13]. 


\subsection{Motivation}

The importance of combustion studies cannot be overemphasized as energy demands have increased across the years. Our society has always depended on energy sources to survive, making it the backbone of civilization. Early civilization used fire for protection during severe cold weather conditions, to ward of predators and to cook. Nowadays, our improved understanding of energy has allowed applications to more sophisticated processes such as automobile transportation and industrial heating. With advancement in technology, our society is on a quest to find new and improved ways to harness and generate energy from alternative/renewable resources such as wind, solar, geothermal. However, among the limitations preventing these kinds of energy sources to be used widely, we should mention in passing associated huge costs and ineffectiveness regarding energy density. Clearly, the fossil fuel energy is not taking a backseat to renewable resources anytime soon as $85 \%$ of all energy we use today comes from combustibles [1]. This sheds the light on the importance of combustion research and the need for the development of next-generation cleaner combustion technologies.

As identified already, two regimes exist for premixed combustion, namely, the deflagration and detonation. Most standard automobiles operate in the deflagration regime but the energy released during the detonation regime is far superior to that of deflagration. Hence, sophisticated engines such as PDEs operating in the detonation regime can revolutionize our industry needs.

Also, understanding of what causes a flame to trigger detonation plays a key role in future prevention of catastrophes in mines and plants. Ideally, the first approach to the mining safety is the complete prevention of an explosion. However, if this is out of control, then understanding of the processes (in order to mitigate the effects of this explosion) is the key factor in saving lives.

The present thesis extends the Bychkov mechanism [7] (focused primarily on equidiffusive flames) to understand the effect of non-equidiffusivity (i.e. non-unity Lewis number, $L e$, which is the thermal-to-mass diffusivity ratio) on FA and whether this factor has an impact on all the previously identified flame acceleration mechanisms.

\subsection{Gap in Knowledge}

It is already well known in the field of combustion that flame acceleration is extremely strong in a tooth-brush-like obstructed channel. However, most of the prior research done in this area was for equidiffusive flames. Similarly, even there were experimental and computational studies on 
the impact of the Lewis number on flame propagation pipes, again, the results were limited, and the application were for an unobstructed geometry. Hence, prior to beginning work on this topic, it was believed that the Lewis impact for this particular configuration was not going to be significant. However, the results obtained proved otherwise, and the acceleration mechanism is indeed as great as that for a non-equidiffusive flame in an unobstructed geometry. 


\section{Chapter 2: Flame Acceleration in Obstructed Channels}

Although FA in obstructed channels was oftentimes attributed to turbulence [4, 5], it is recalled that there exists a conceptually laminar mechanism of FA in obstructed pipes, with turbulence and shock waves playing only supplementary roles. This shockless mechanism was revealed by Bychkov et al [7-9], and Figure 2.6 shows a schematic of the geometry considered. Specifically, we have an obstructed tube or channel, filled with a premixture; one extreme (right) of the conduit is open while the other end (left) is kept closed. A flame embryo is ignited at the closed end, and it propagates towards the open one. The channel is free along the centerline, while the obstructions are nearby the walls and are represented by an array of thin, tightly-spaced obstacles resembling a tooth-brush or comb. Here $\Delta z$ is the spacing between the obstacles, and the obstacles height is $\alpha R$ such that if $R$ is a channel radius (half-width), then quantity $\alpha$ is the blockage ratio. This is a key parameter of the problem, as it shows the fraction of the channel occupied by the obstacles.

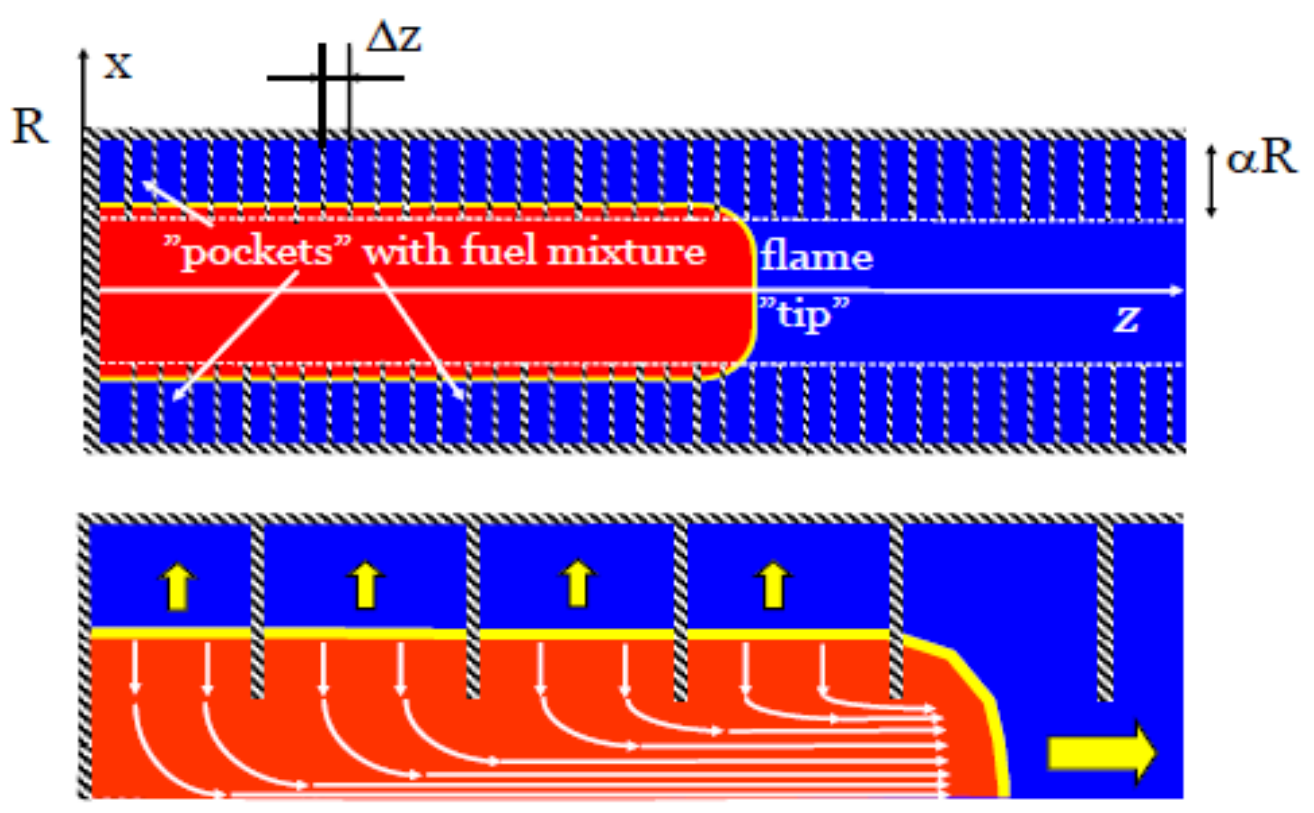

Figure 2.6 - Laminar mechanism of flame acceleration in a semi-open channel filled with obstacle [8]

The Bychkov acceleration mechanism works as follows. Combustion generates a new volume, due to thermal expansion of the burning matter, such that flame propagation acts as a piston pushing a flow towards the fuel-air mixture. Some portion of this fresh mixture appears trapped in the "pockets" between the obstacles, while the flow near the centerline propagates freely and fast. This leads to a delay in the combustion of the mixture in the pockets, which invariably drifts 
the flame, creating new pockets. This increased flow is forced towards the centerline of the channel, accelerating the flame downstream of the channel. Consequently, we have a positive feedback between the flame and the flow, promoting each other. Namely, a jet flow emerges and it drives FA. The velocity of this flame tip in the laboratory reference frame, $U_{t i p}$, obeys the formula [7]

$$
\frac{U_{t i p}}{S_{L}} \sim \Theta \exp \left(\frac{\sigma t S_{L}}{R}\right),
$$

where $S_{L}$ is the unstretched laminar flame speed (if a flame front was planar, then it would propagate in the same medium with the velocity $S_{L}$ with respect to the fuel-air mixture) and $\Theta=\rho_{\text {unburnt }} / \rho_{\text {burnt }}$ is the thermal expansion ratio.

Equation (2.1) yields exponential FA, with an exponential acceleration rate $\sigma$. According to Ref. [7], the quantity $\sigma$ is a function of the thermal expansion factor $\Theta$ and the blockage ratio $\alpha$,

$$
\sigma=\frac{\Theta-1}{1-\alpha},
$$

but it does not depend on other parameters such as the obstacles spacing $\Delta z$ and the channel halfwidth $R$. This makes the Bychkov mechanism Reynolds-independent and thereby employable to a variety of scales and applications, ranging from small micro tubes to large tunnels. 


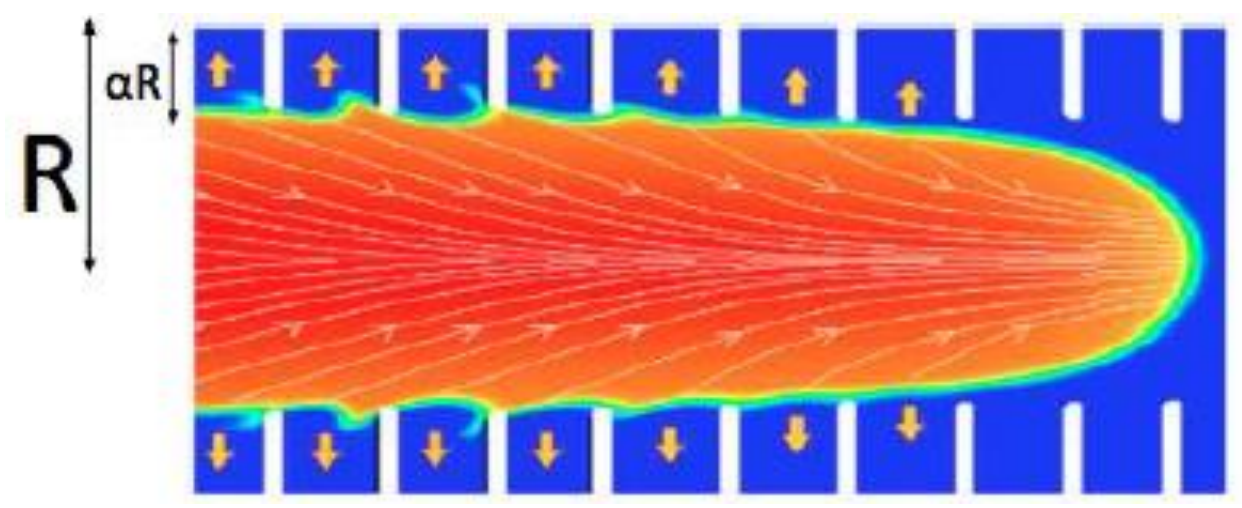

Figure 2.7 -A color snapshot from a numerical simulation of FA in a channel with thinly packed obstacles[9]

The Bychkov theory of FA in such an obstructed configuration is presented above; see, for instance, Refs. [7,8] for more details. Once burning is completed in a pocket, the burnt gas exits the pocket with a flow speed $\left|u_{x}\right|=(\Theta-1) S_{L}$. This burnt matter adds new volume to the unobstructed region of the channel thereby causing a jet-flow that pushes a flame downstream, accelerating it. At the initial stage of acceleration, the gas mixture is assumed to be incompressible, thereby obeying the continuity equation in the form $\nabla \cdot \boldsymbol{u}=0$.

With the geometry defined as the flame propagation from the closed end to the open end, the velocity at the closed end is $u_{z}=0$ considering that $z=0$ at the closed end. Hence the velocity distribution in the center region without obstacles for $|x|<(1-\alpha) R$ reads (see also $[7,8])$ :

$$
u_{x}=-\frac{(\Theta-1)}{(1-\alpha)} \frac{S_{L}}{R} x, \quad u_{z}=\frac{(\Theta-1)}{(1-\alpha)} \frac{S_{L}}{R} z
$$

Indeed, the flame gains strength as it approaches the open end of the channel due to the fact that several pockets would have contributed to the jet flow. Then, the flame tip position $Z_{f}$ obeys the equation

$$
\frac{d Z_{f}}{d t}=u_{z}\left(Z_{f}\right)+\Theta S_{L}=\frac{(\Theta-1) S_{L}}{(1-\alpha) R} Z_{f}+\Theta S_{L}
$$

with the solution

$$
\frac{Z_{f}}{(1-\alpha) R}=\frac{\Theta}{\Theta-1}\left[\exp \left(\sigma S_{L} t / R\right)-1\right]
$$


where the exponential factor is given by Eq. (2.2), $\sigma=(\Theta-1) /(1-\alpha)$. Obviously, $\alpha<1$ such that $\sigma>0$. It is seen from Eqs. (2.2), (2.5) that both increases in the thermal expansion and the blockage ratio strongly amplify $\sigma$ thereby promoting flame acceleration.

It is noted that the Bychkov theory and modelling [7-9] employed a conventional approach of equidiffusivity of a fuel-air mixture. This means that the Lewis number was unity

$$
\text { Le } \equiv \text { Thermal Diffusivity/Mass Diffusivity }=1 .
$$

While Eq. (2.6) is a classical, adoptable approach in theoretical works, the Lewis number may noticeably deviate from unity in the practical reality, which leads to intriguing effects such as the onset of diffusional-thermal instability (primarily, for $L e<1$ flames) and flame thickening (for $L e<1$ flames). Consequently, there has been a critical need to identify and quantify the impacts of non-equidiffusivity (non-unity $L e$ ) on the flame dynamics in obstructed channels, and this question is addressed in the present work by means of comprehensive computational simulations. 


\section{Chapter 3: Methodology}

A fully-compressible, finite-volume Navier-Stokes solver available at Dr. Akkerman's group at WVU served as the computational platform for the simulations performed. The code is formulated to solve the set of the hydrodynamic and combustion equations, including transport processes (diffusion, viscosity, and heat conduction) and an Arrhenius chemical kinetics. The numerical scheme is $2^{\text {nd }}$-order accurate in time, $4^{\text {th }}$-order in space for the convective terms, and $2^{\text {nd }}$-order in space for the diffusive terms. The embryo of this code was originally developed at Volvo Aero, Sweden, and it has subsequently been updated, comprehensively, by the research groups of Chalmers University of Technology, Royal Institute of Technology, Uppsala and Umeå Universities in Sweden as well as Princeton University and West Virginia University in the U.S. The code is robust and accurate, having been successfully utilized in numerous aeroacoustic and combustion applications. The computational time for each job run on the cluster is for about 300 hours, with post-processing efforts needed once a simulation is completed. The solver is adapted for parallel computations and available in the 2D versions (Cartesian and cylindrical axisymmetric ones) as well as a fully 3D Cartesian version, with a self-adaptive structured computational grid employed that makes the code perfect, in particular, for fundamental studies of flame hydrodynamics in combustors of high aspect ratios.

The present thesis is limited to the $2 \mathrm{D}$ consideration. Then the basic equations are:

- the continuity equation:

$$
\frac{\partial \rho}{\partial t}+\frac{\partial}{\partial x_{i}}\left(\rho u_{i}\right)=0
$$

- the momentum equation:

$$
\frac{\partial}{\partial t}\left(\rho u_{i}\right)+\frac{\partial}{\partial x_{i}}\left(\rho u_{i} u_{j}+p \delta_{i j}-\gamma_{i j}\right)=0,
$$

- the energy equation:

$$
\frac{\partial}{\partial t}\left(\rho \varepsilon+\frac{1}{2} \rho u_{i} u_{j}\right)+\frac{\partial}{\partial x_{i}}\left(\rho u_{j} H+\frac{1}{2} \rho u_{i} u_{i} u_{j}+q_{j}-u_{i} \gamma_{i j}\right)=0,
$$

- and the species equation:

$$
\frac{\partial}{\partial t}(\rho Y)+\frac{\partial}{\partial x_{i}}\left(\rho u_{i} Y-\frac{\zeta}{S_{c}} \frac{\partial Y}{\partial x_{i}}\right)=-\frac{\rho Y}{\tau_{R}} \exp \left(-E_{A} / R_{u} T\right) .
$$

Equation (3.4) describes a single irreversible Arrhenius reaction of the first order, with activation energy $E_{a}$ and constant of time dimension $\tau_{R}$. In the set of equations above, the specific internal 
energy $\varepsilon$ and enthalpy $h$ are given by $\varepsilon=Q Y+C_{V} T$ and $h=Q Y+C_{P} T$, where $Y$ is the mass fraction of the fuel, $T$ is the temperature, $C_{V}$ and $C_{p}$ are specific heats and $Q=C_{P} T_{f}(\Theta-1)$ is the energy released in the reaction. The stress tensor $\gamma_{i j}$ and the energy diffusion vector $q_{i}$ are

$$
\gamma_{i j}=\zeta\left(\frac{\partial u_{i}}{\partial x_{j}}+\frac{\partial u_{j}}{\partial x_{i}}-\frac{2}{3} \frac{\partial u_{k}}{\partial x_{k}} \delta_{i j}\right), \quad q_{i}=-\zeta\left(\frac{C_{P}}{\operatorname{Pr}} \frac{\partial T}{\partial x_{j}}+\frac{Q}{S c} \frac{\partial Y}{\partial x_{j}}\right),
$$

where $\zeta=1.7 \times 10^{-5} \mathrm{~kg} / \mathrm{m} \mathrm{s}$ is the dynamic viscosity in the fuel mixture and $\operatorname{Pr}$ and $S c$ are the Prandtl and Schmidt numbers, respectively. It is noted that their ratio provides the Lewis number $L e=S c / P r$ (compare to Eq. (2.6)) - the major parameter of the present work controlling nonequidiffusivity; it has been varied in the range $0.2 \leq L e \leq 2.0$. Sc was varied while $\operatorname{Pr}$ was held constant at 1 .

The initial fuel properties were taken as large as: temperature $T_{f}=300 \mathrm{~K}$, pressure $P_{f}=$ 1 bar, and density $\rho_{f}=1.16 \mathrm{~kg} / \mathrm{m}^{3}$. The thermal expansion ratio was taken to be $\Theta=8$, with the laminar flame speed being $S_{L}=34.7 \mathrm{~cm} / \mathrm{s}$, which is $10^{3}$ times less than the initial speed of sound $c_{0}=347 \mathrm{~m} / \mathrm{s}$, making the hydrodynamics to be almost incompressible at the initial stage of burning, with the conventional flame Mach number being $M_{0} \equiv S_{L} / c_{0}=0.001 \ll 1$. The instantaneous flame tip Mach number, $M_{t i p}(t)$, is introduced as the instantaneous flame tip velocity $U_{t i p}(t)$, scaled by the instantaneous speed of sound, $c_{t i p}(t), M_{t i p} \equiv U_{t i p} / c_{t i p}$. This parameter is found to be a very good measure of compressibility and also sheds light on the current stage of the DDT process. $M_{\text {tip }}(t)$ was used as a measure of how close the flame was to the onset of detonation, with $M_{\text {tip }} \ll 1$ representing the initial, quasi-isobaric stage of burning and $M_{t i p} \cong 1$ associated with the detonation triggering.

The flame thickness is conventionally defined as $L_{f}=v / \operatorname{Pr} S_{L}=4.22 \times 10^{-5} \mathrm{~m}$. The latter value is the natural scaling for the channel half-width $R$ such that we have the flame propagation Reynolds number in the form $R e=R S_{L} / v=R / \operatorname{Pr} L_{f}=R / L_{f}$. Specifically, $R e=24,36,48$ was considered in this work. Obviously, the laminar flame speed $S_{L}$ constitutes a natural scaling to measure velocities, and in this light we intrinsically arrive to the scaled measure of time in the form $\tau=S_{L} t / R$. It is recalled that our computational domain is set-up in such a manner such that a flame propagates in a long $2 \mathrm{D}$ channel of width $2 R$, with fraction of the channel $\alpha$ blocked by the obstacles (such that the obstacle length is $2 R \alpha$ ), and with the obstacle spacing $\Delta z$; see Figures 2.6 and 2.7. Consequently, the other dimensionless parameters of the problem are the 
scaled spacing $\Delta z / R$ (here I used $\Delta Z / R=0.25$ ) and the blockage ratio $\alpha$ (the cases $\alpha=1 / 3$, $1 / 2,2 / 3$ were considered).

The boundary conditions at the walls were: free-slip, i.e. wall friction is ignored, $\boldsymbol{n} \cdot \boldsymbol{u}=\mathbf{0}$, with $\boldsymbol{n}$ indicating the normal vector at a wall, and adiabatic, i.e. $\boldsymbol{n} \cdot \nabla \mathrm{T}=0$. As for the extremes of the channel, a semi-open channel was chosen, with the closed left end, where the medium was initially at rest, and the open right extreme, where the absorbing (non-reflecting) boundary conditions are applied to prevent the reflection of shock waves from the open exit. Both the fuelair mixture and the burnt matter are assumed to be ideal gases of constant molecular weight $W_{M}=29 \mathrm{~kg} / \mathrm{kmol}$ such that the equation of state reads $P=\rho R_{u} T / W_{M}$, with the universal gas constant $R_{u}=8.31 \mathrm{~kJ} / \mathrm{kmol} \cdot \mathrm{K}$. 


\section{Chapter 4: Results and Discussion}

Comprehensible computational simulations have been performed to identify the impacts of $L e, \alpha$ and $R e$ on the dynamics and morphology of accelerating flames in "tooth-brush"-like obstructed pipes as illustrated in Figures 2.6 and 2.7. The results are summarized and discussed below.

\subsection{Flame front snapshots}

To better visualize the FA mechanism in the obstructed geometry, color temperature snapshots showing the flame shape at the specified time instance are presented in Figure 4.8 and analyzed.
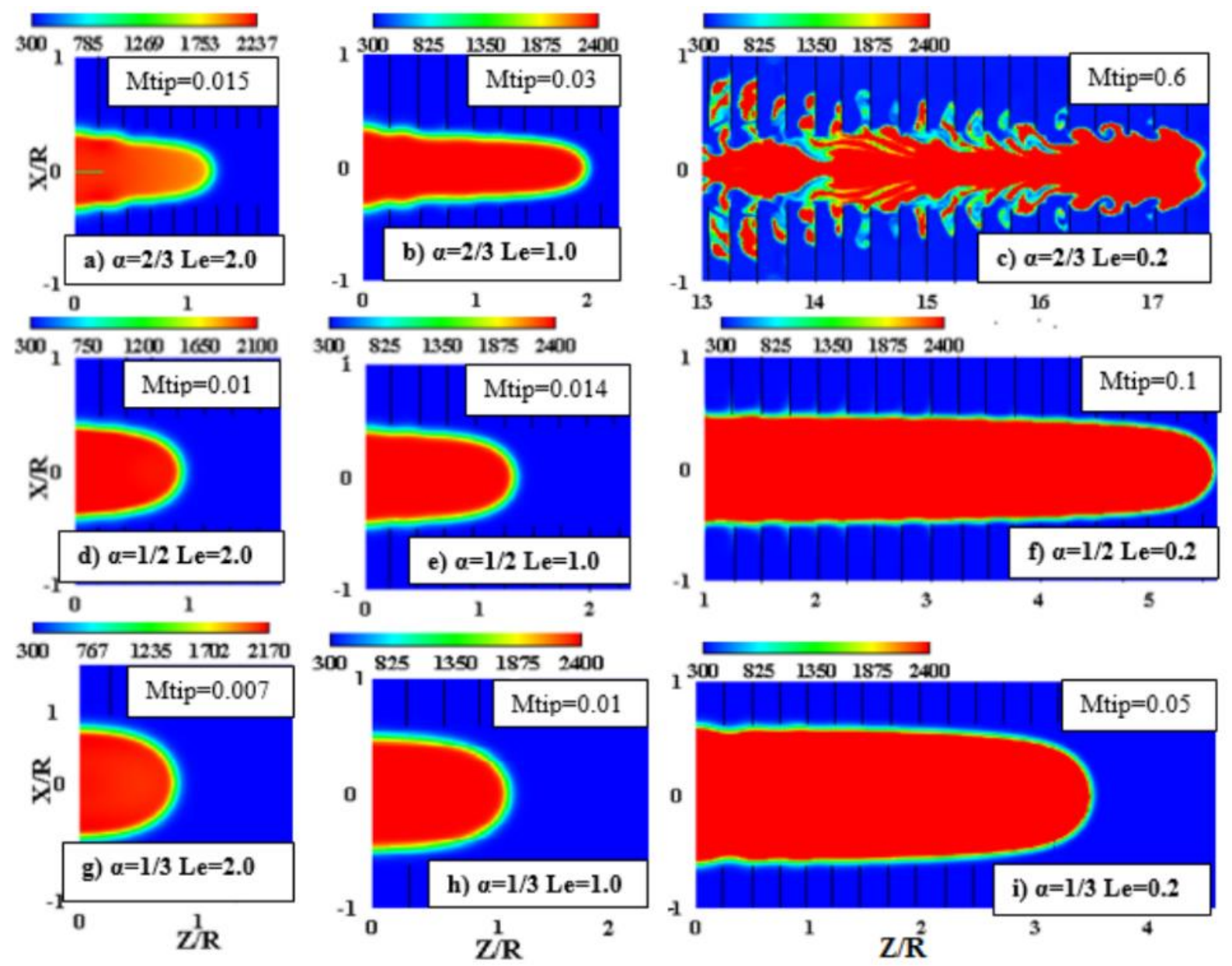

Figure 4.8 - Temperature snapshot [from $300 \mathrm{~K}$ in the fuel mixture (blue) to $2400 \mathrm{~K}$ in the burnt matter] at the same time instant i.e. scaled time, $\tau=0.075$ for $\operatorname{Re}=24$ and various Le and $\alpha$

Figure 4.8 (a-i) shows the flame image for various $L e, L e=0.2,1.0,2.0$ and various $\alpha, \alpha=$ $1 / 3,1 / 2,2 / 3$ at the same scaled time instant, $\tau=0.075$ for the same $R e, R e=24$ in all cases. For all these runs, the flame tip Mach number $M_{\text {tip }}$ is also recorded and shown. Obviously, the blue color corresponds to the cold, fresh fuel mixture while the burnt matter is shown by red, and 
the flame is between them. The impact of the Lewis number is evidently seen to be enormous and it shows to be as strong as that of blockage ratio. When the effect of blockage ratio is smallest and the flame is non-equidiffusive with $L e>1$ as shown in Figure $4.8 \mathrm{~g}$, the flame experiences the slowest acceleration with a corresponding $M_{t i p}=0.007$. In contrast, when the blockage ratio is largest and $L e>1$ (Figure 4.8c) the coupling of both effects causes the flame to accelerate rapidly and approach detonation, with a flame tip Mach number as high as $M_{\text {tip }}=0.6$. In other cases of Figure 4.8, the effects of $\alpha$ and $L e$ compete such that what is observed is an almost equivalent flame in the "pairs" $4.8 \mathrm{~d}$ and 4.8h; 4.8a and 4.8e; and even 4.8b and 4.8i, respectively. In these cases, both factors strive to influence the flame propagation and contribute to the similarity in the paired results.

As a result, the very first figure, Figure 4.8, allows anticipating that the impact of Lewis number will be at least comparable to that of the major parameter characterizing flame propagation in obstructed tooth-brushed conduits - the blockage ratio $\alpha$, which is scrutinized next in detail

\subsection{Effect of Lewis number on flame acceleration}

To evaluate the impact of Le, the time evolution of the scaled flame tip velocity $U_{\text {tip }} / S_{L}$ has been analyzed for fixed flame $\operatorname{Re}, \operatorname{Re}=24,36,48$ and fixed $\alpha, \alpha=1 / 3,1 / 2,2 / 3$. Specifically, Figures 4.9(a-c) compare the scaled flame tip velocities for various $L e=$ 0.2, 1.0, 2.0 in each figure, with fixed $\alpha=1 / 3$, in Figure 4.9a, $\alpha=1 / 2$ in Figure 4.9b, and $\alpha=2 / 3$ in Figure 4.9c; with fixed $R e=R / L_{f}=24$ in all cases of Figure 4.9. Figures 4.10(a-c) are the complete counterparts of Figure 4.9(a-c), but for a wider channel, $R e=36$. Eventually, Figures 4.11(a-c) are the complete counterparts of Figures 4.9 and 4.10, but for an even wider channel, with $R e=48$.

It is seen from Figures 4.9-4.11 that the effect of $L e$ is very strong indeed, especially for Le $<1$ flames. Indeed, in all nine plots, Figures 4.9(a-c)-4.11(a-c), a small Lewis number, $L e=0.2$, promotes $U_{\text {tip }}$ almost by an order of magnitude as compared to the equidiffusive case, $L e=1$. This effect is devoted to the onset of the diffusional-thermal instability. As for $L e>1$ flames, here the effect is much weaker, but $L e=2.0$, nevertheless, noticeably moderates FA as compared to the $L e=1$ case. This, presumably, happens due to the flame thickening such that it becomes harder for a flame front to get corrugated in the same channel as for $L e=1$. 


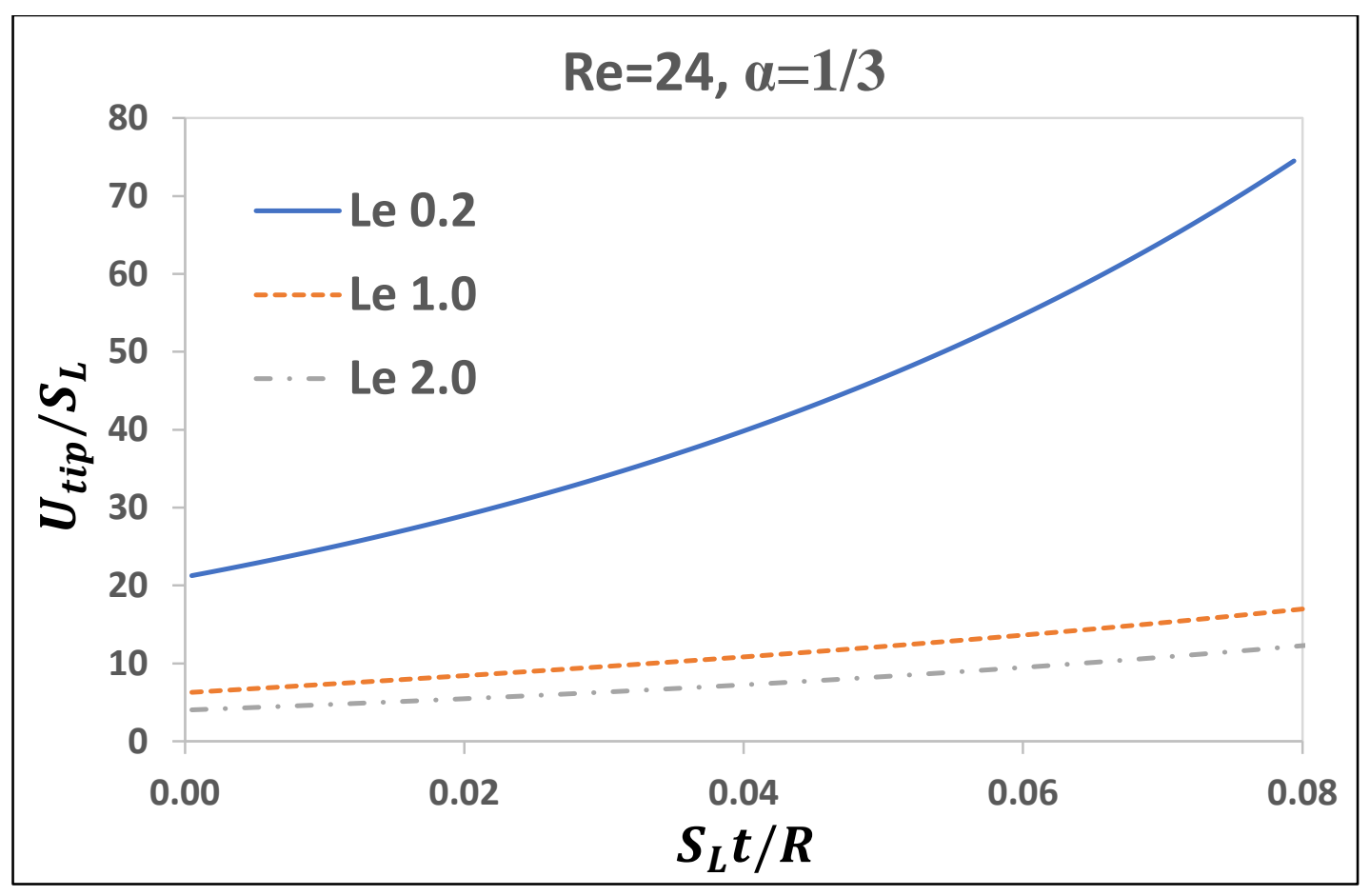

Figure $4.9 a$ - Scaled flame tip velocity as a function of scaled time for fixed $R e=24$ and $\alpha=1 / 3$

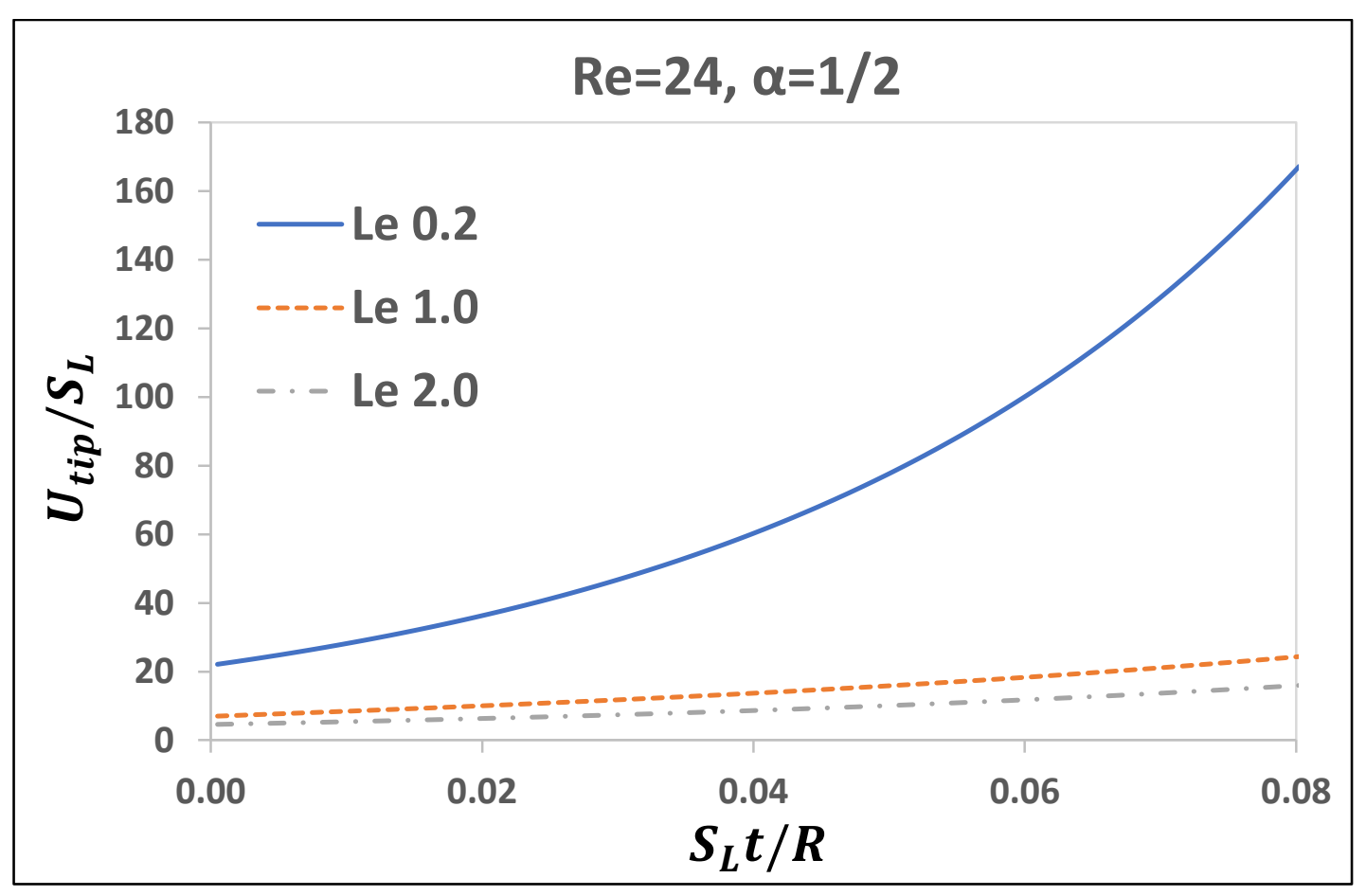

Figure $4.9 b$ - Scaled flame tip velocity as a function of scaled time for fixed $R e=24$ and $\alpha=1 / 2$ 


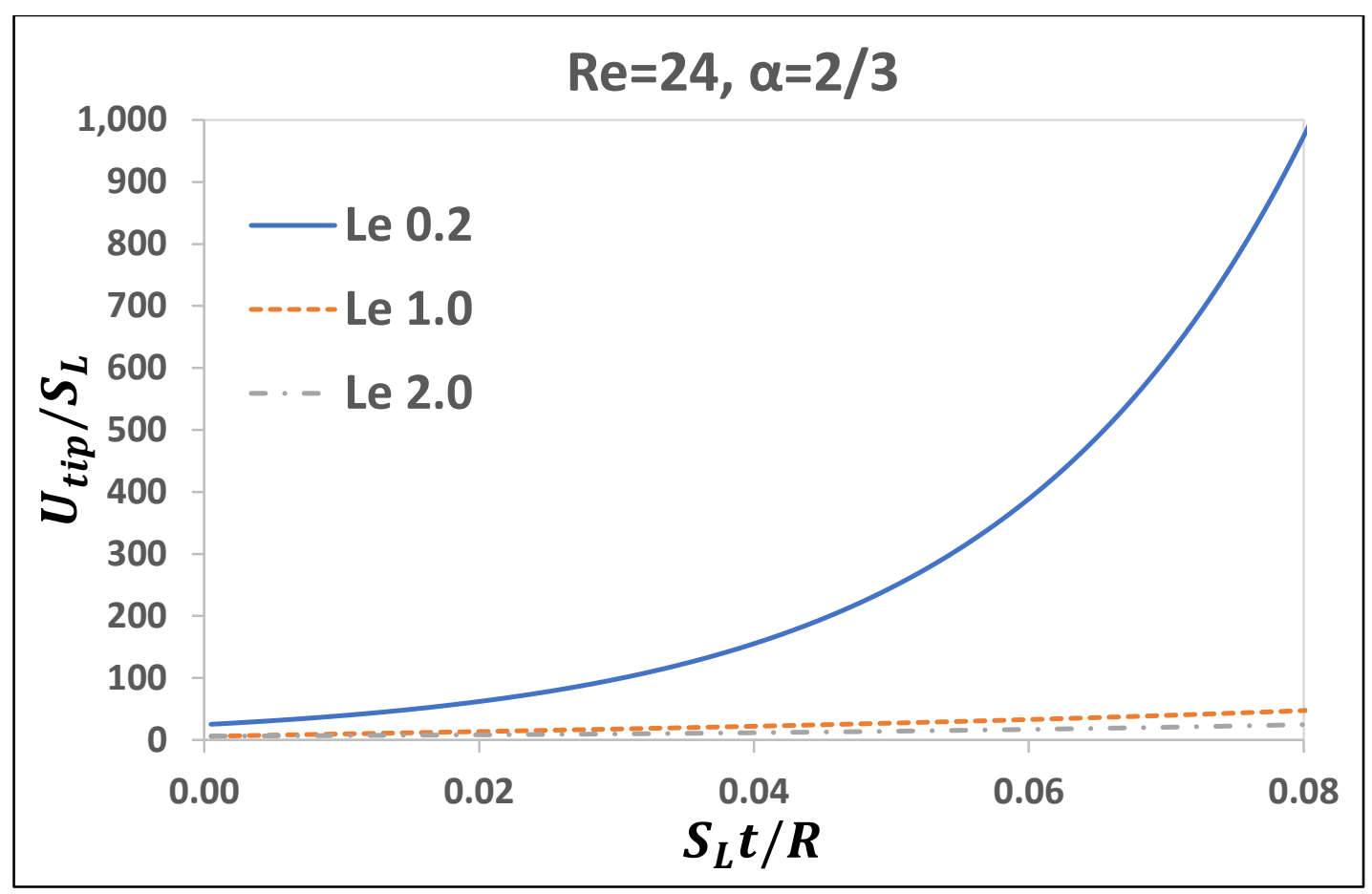

Figure 4.9c-Scaled flame tip velocity as a function of scaled time for fixed $R e=24$ and $\alpha=2 / 3$

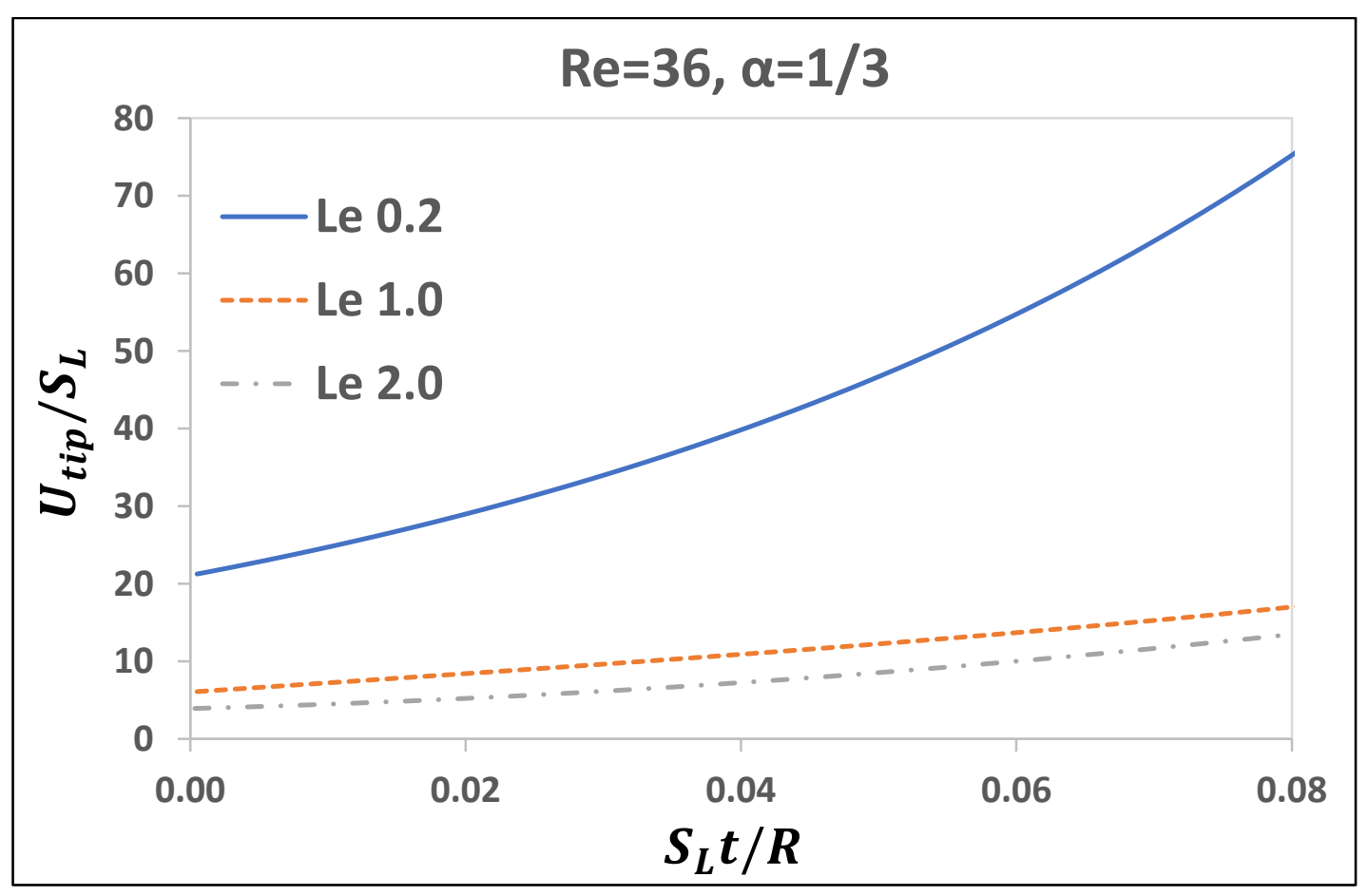

Figure 4.10a - Scaled flame tip velocity as a function of scaled time for fixed Re=36 and $\alpha=1 / 3$ 


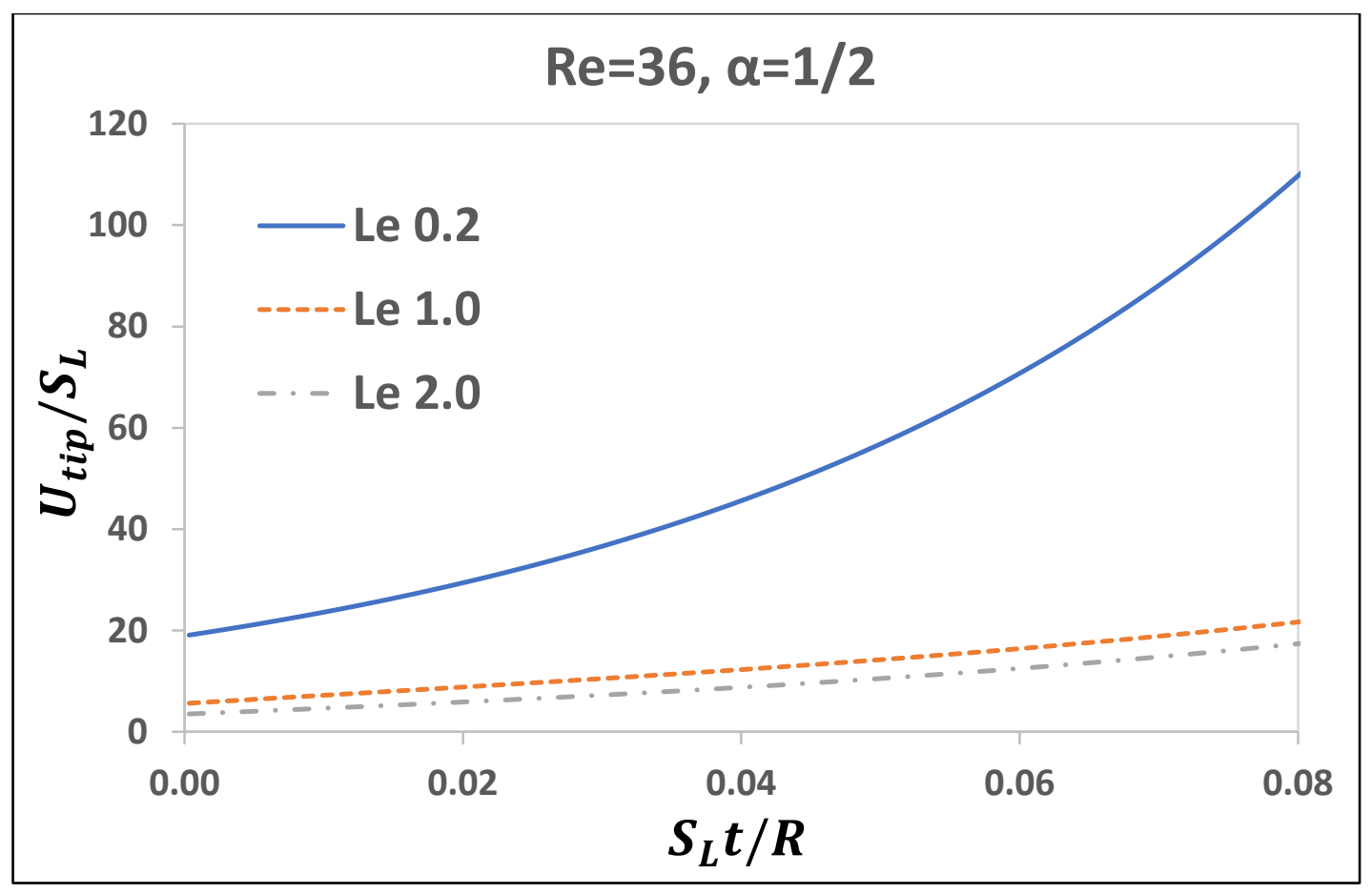

Figure 4.10b-Scaled flame tip velocity as a function of scaled time for fixed $R e=36$ and $\alpha=1 / 2$

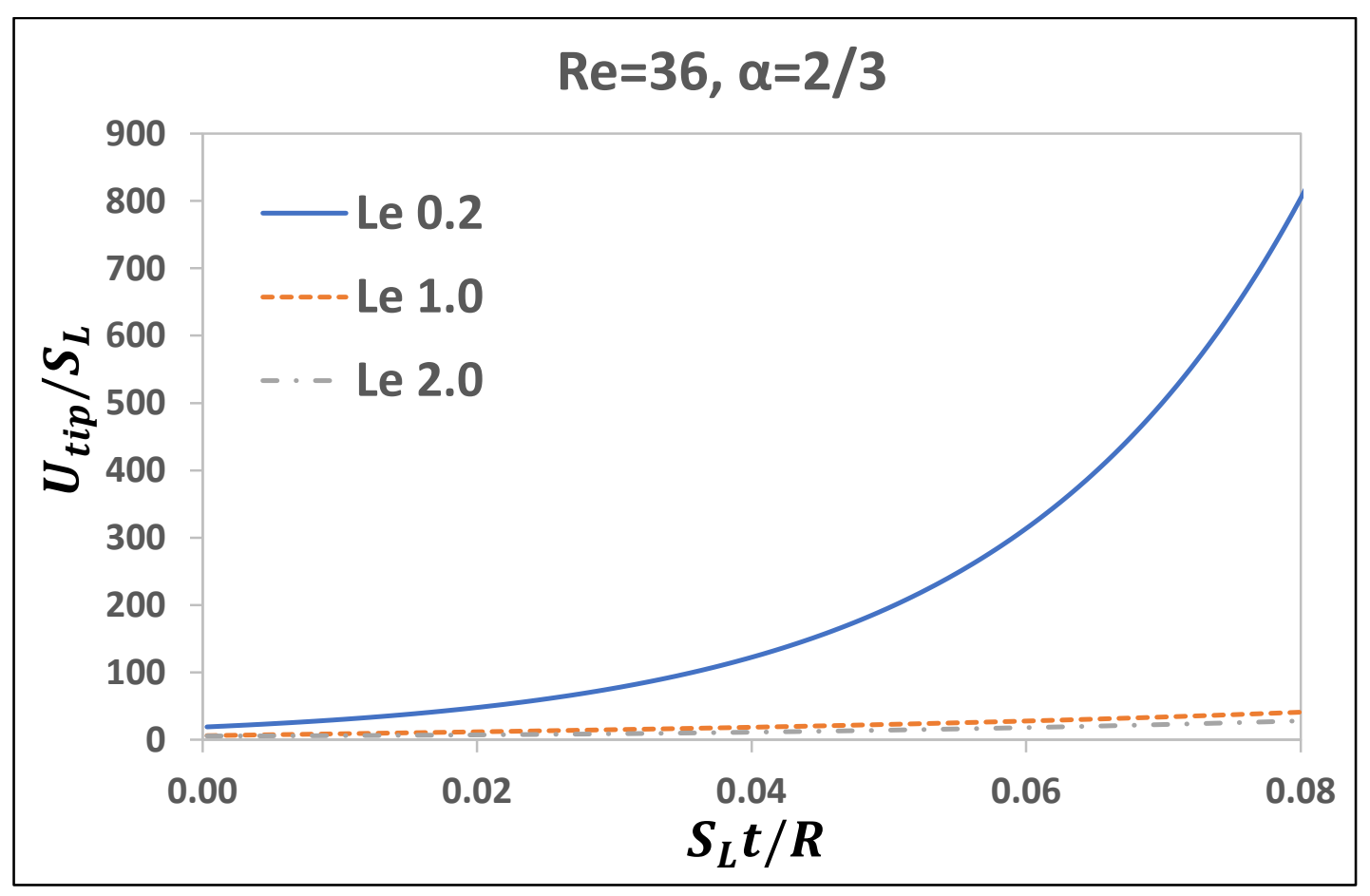

Figure 4.10c-Scaled flame tip velocity as a function of scaled time for fixed $R e=36$ and $\alpha=2 / 3$ 


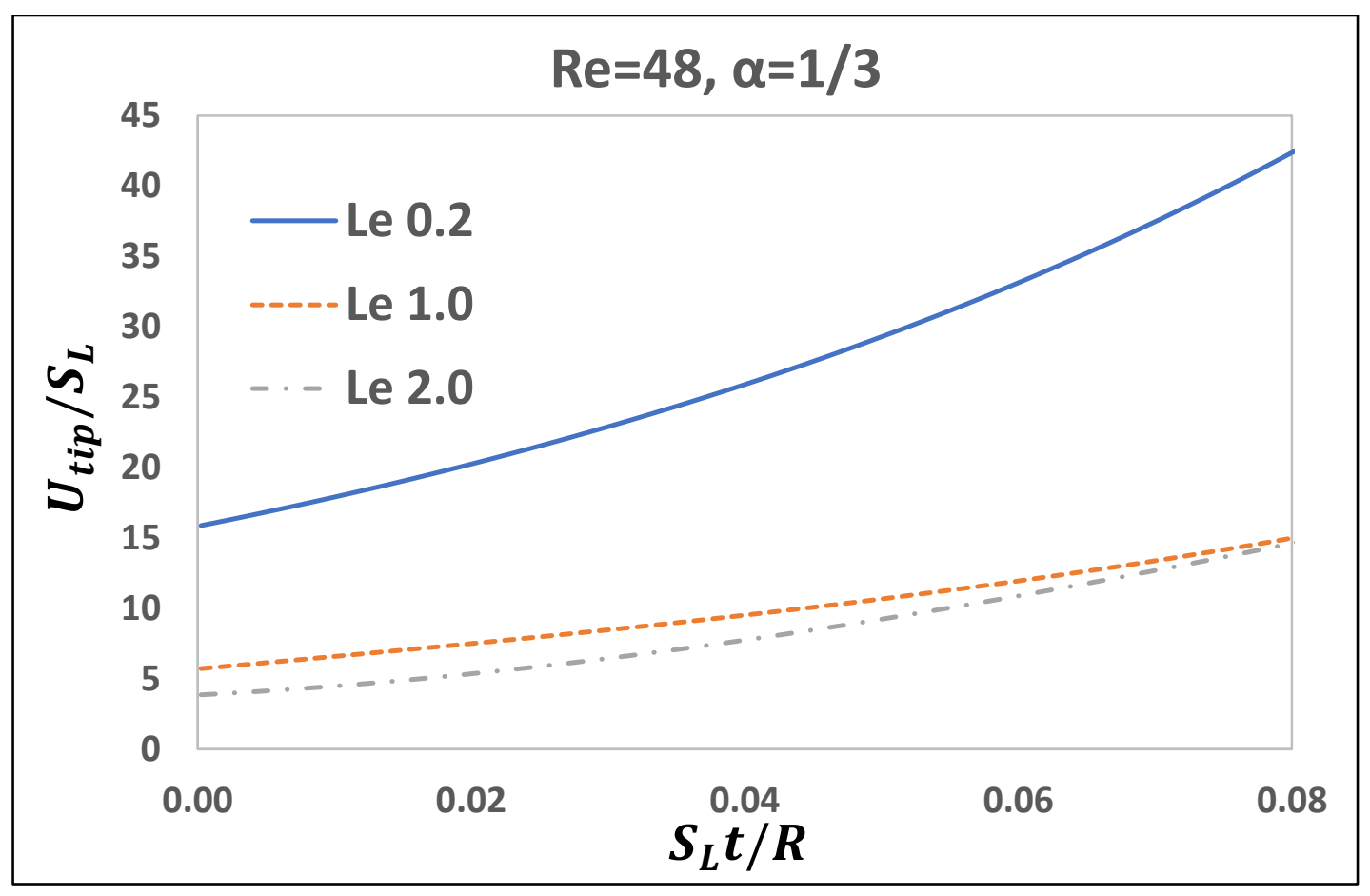

Figure 4.11a-Scaled flame tip velocity as a function of scaled time for fixed $R e=48$ and $\alpha=1 / 3$

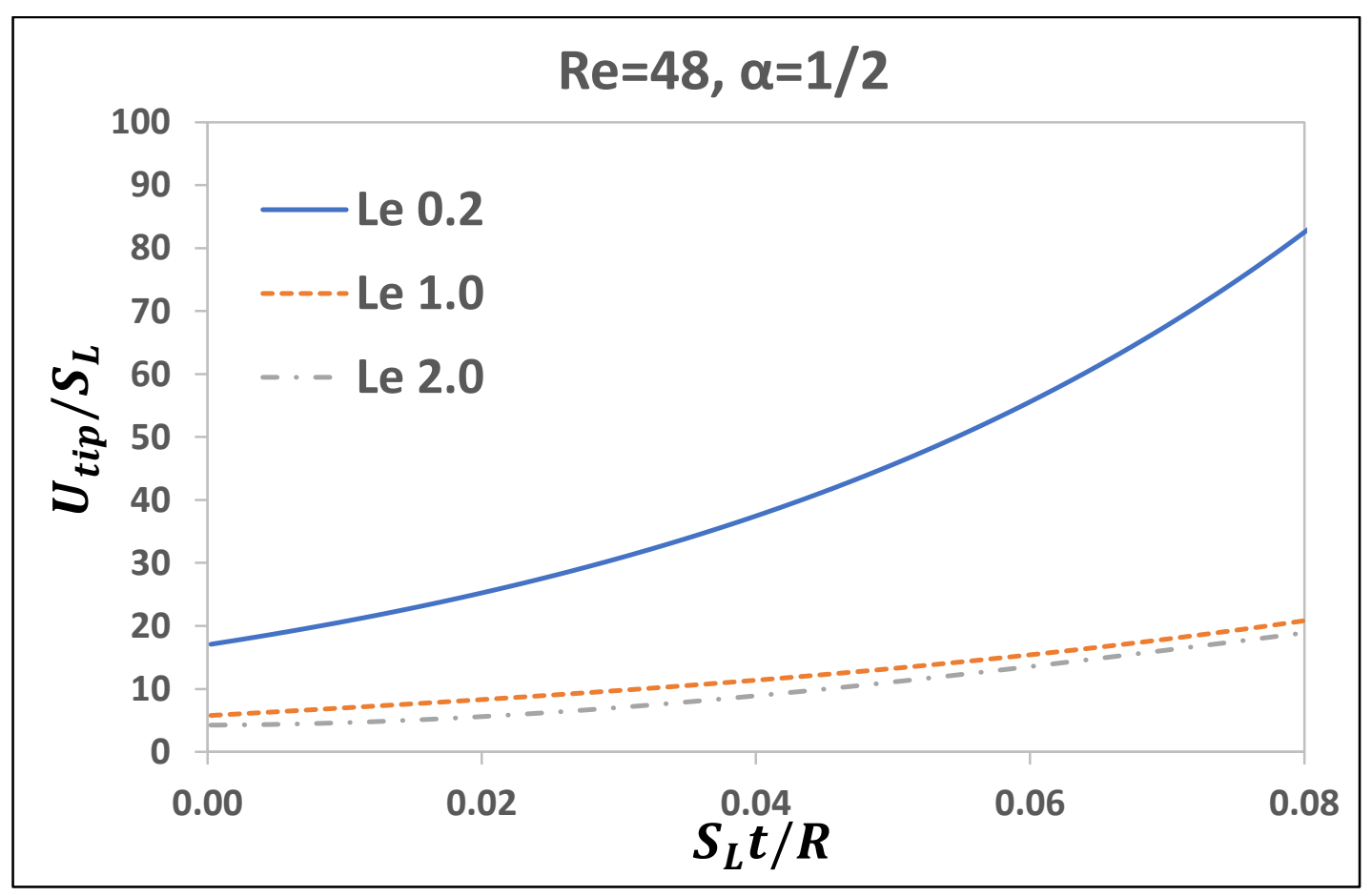

Figure $4.11 b$ - Scaled flame tip velocity as a function of scaled time for fixed Re=48 and $\alpha=1 / 2$ 


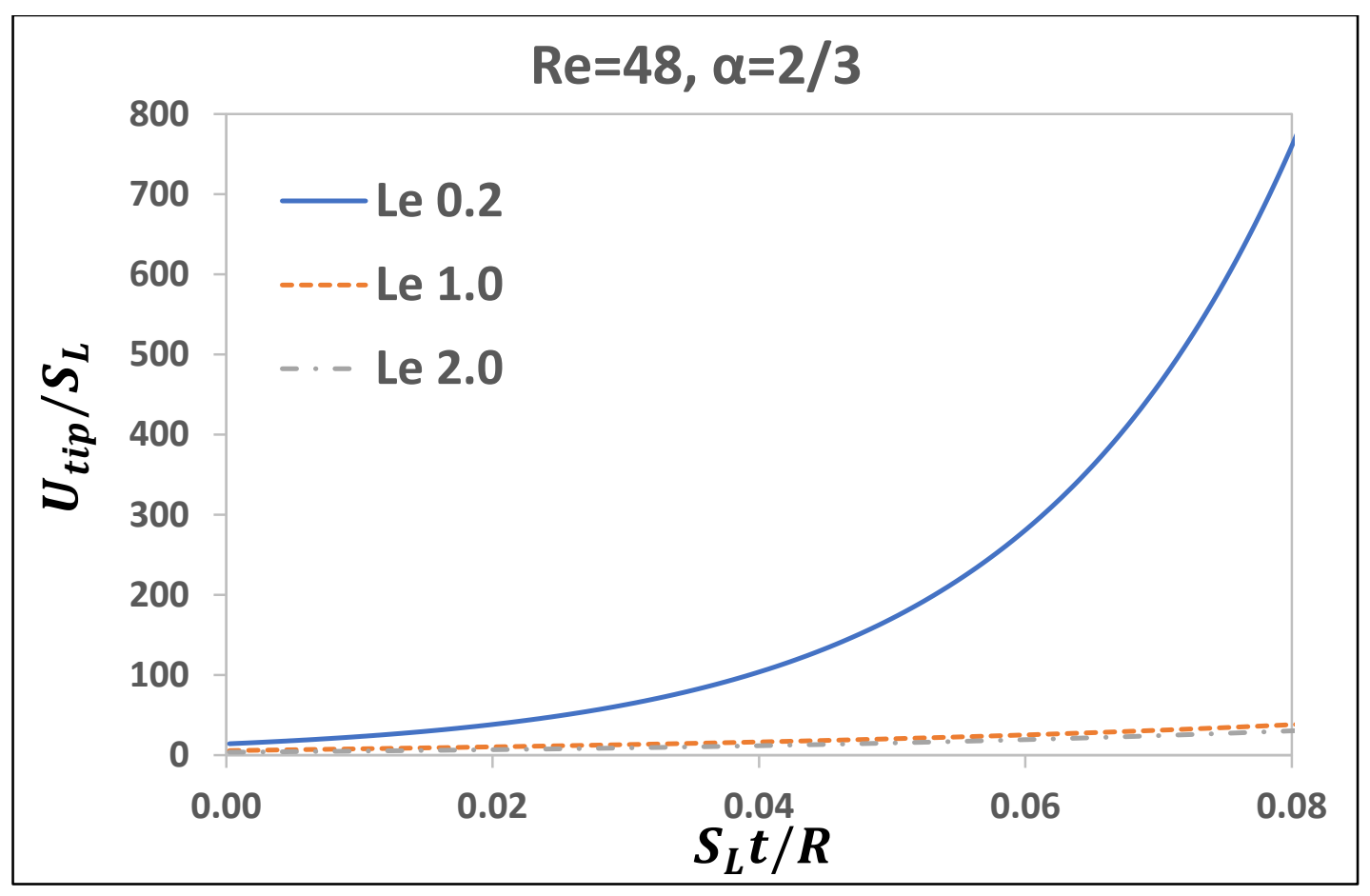

Figure 4.11c-Scaled flame tip velocity as a function of scaled time for fixed $R e=48$ and $\alpha=2 / 3$

Figure 2-4.14 show the flame evolution by means of the temperature snapshots taken at the scaled time instants $\tau=0.043(a), 0.063(b), 0.078(c)-$ for $L e=0.2$ in Figure 4.12, $L e=1.0$ in Figure 4.13 and $L e=2.0$ in Figure 4.14. In all these cases of Figures 4.12-4.14, the same (wide) channel, $R e=48$, and the same (high) blockage ratio, $\alpha=2 / 3$ was employed. It is seen that flames propagate in the fastest manner in the case of lowest Lewis number, $L e=0.2$, which is devoted to the diffusional-thermal instability of non-equidiffusive $(L e<1)$ flames. Indeed, the $L e=0.2$ flame appears to be dramatically unstable and its shape is drastically fold; at $\tau=0.078$ it has propagated almost 15 times faster than the respective equidiffusive ( $L e=1)$ flame. The effect of $L e>1$ is smaller: The flame evolutions (the color snapshots) of Figures 4.13 and 4.14 are qualitatively similar, though - quantitatively - the Le $=2.0$ flame in Figure 4.14 propagates slower than the equidiffusive flame in Figure 4.13. 
(a)

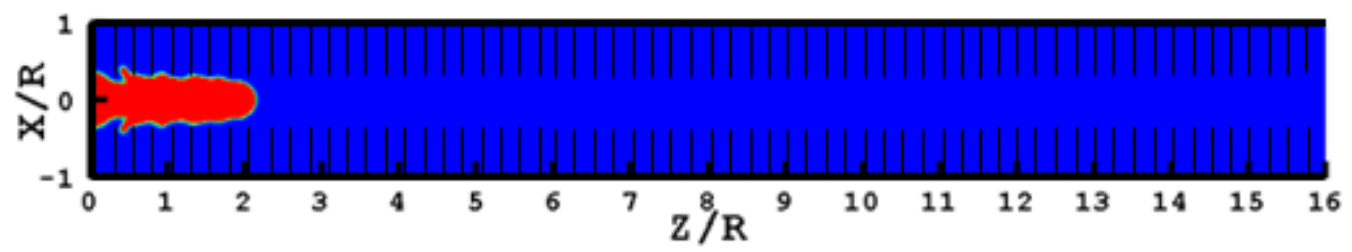

(b)

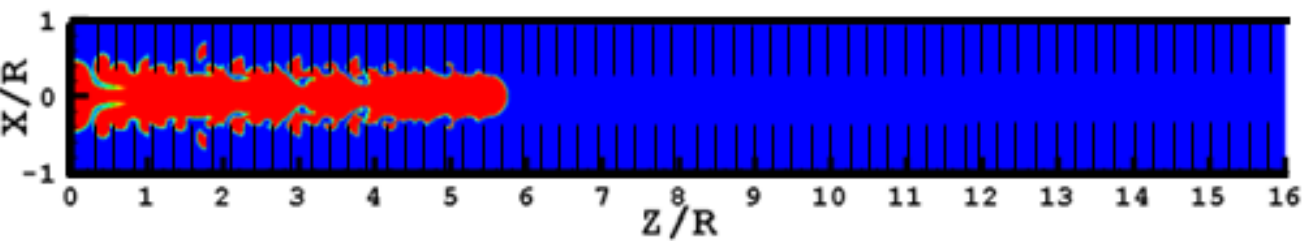

(c)

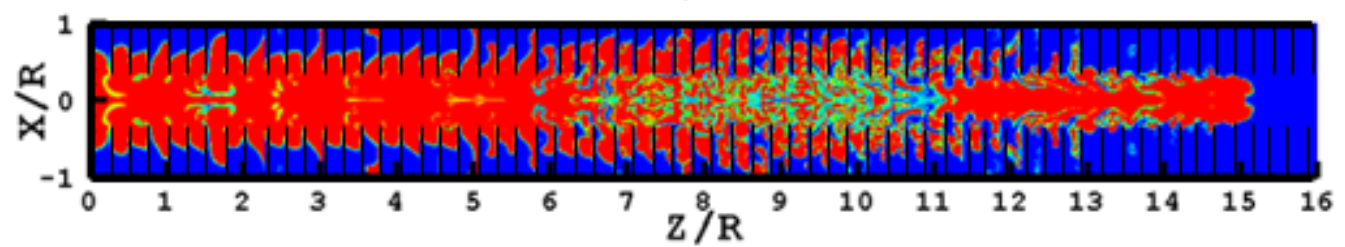

Figure 4.12-Temperature profiles for flames with $R e=48, \alpha=2 / 3$ and Le=0.2 for various scaled times $\tau=0.043(a) 0.063(b)$ $0.078(c)$
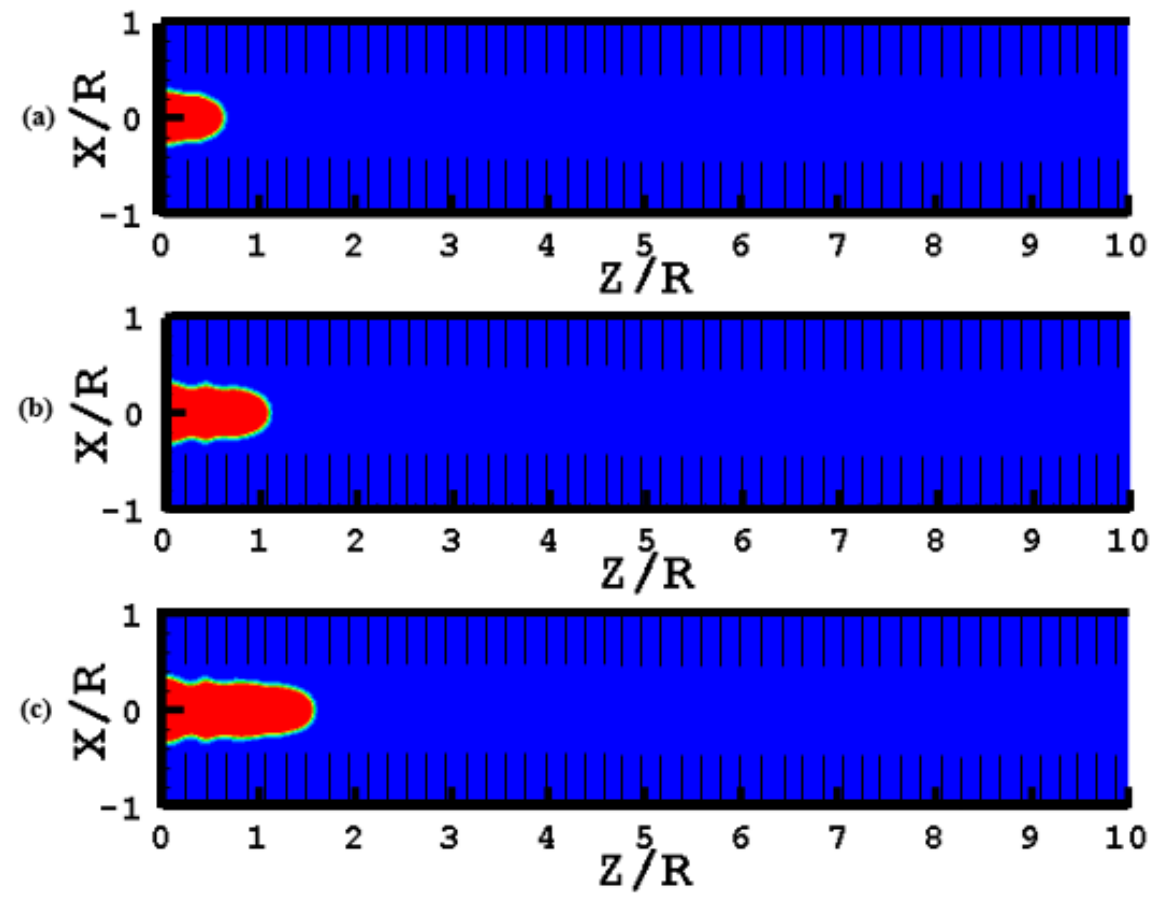

Figure 4.13-Temperature profiles for flames with $R e=48, \alpha=2 / 3$ and Le $=1.0$ for various scaled times $\tau=0.043(a) 0.063$ (b) $0.078(c)$ 

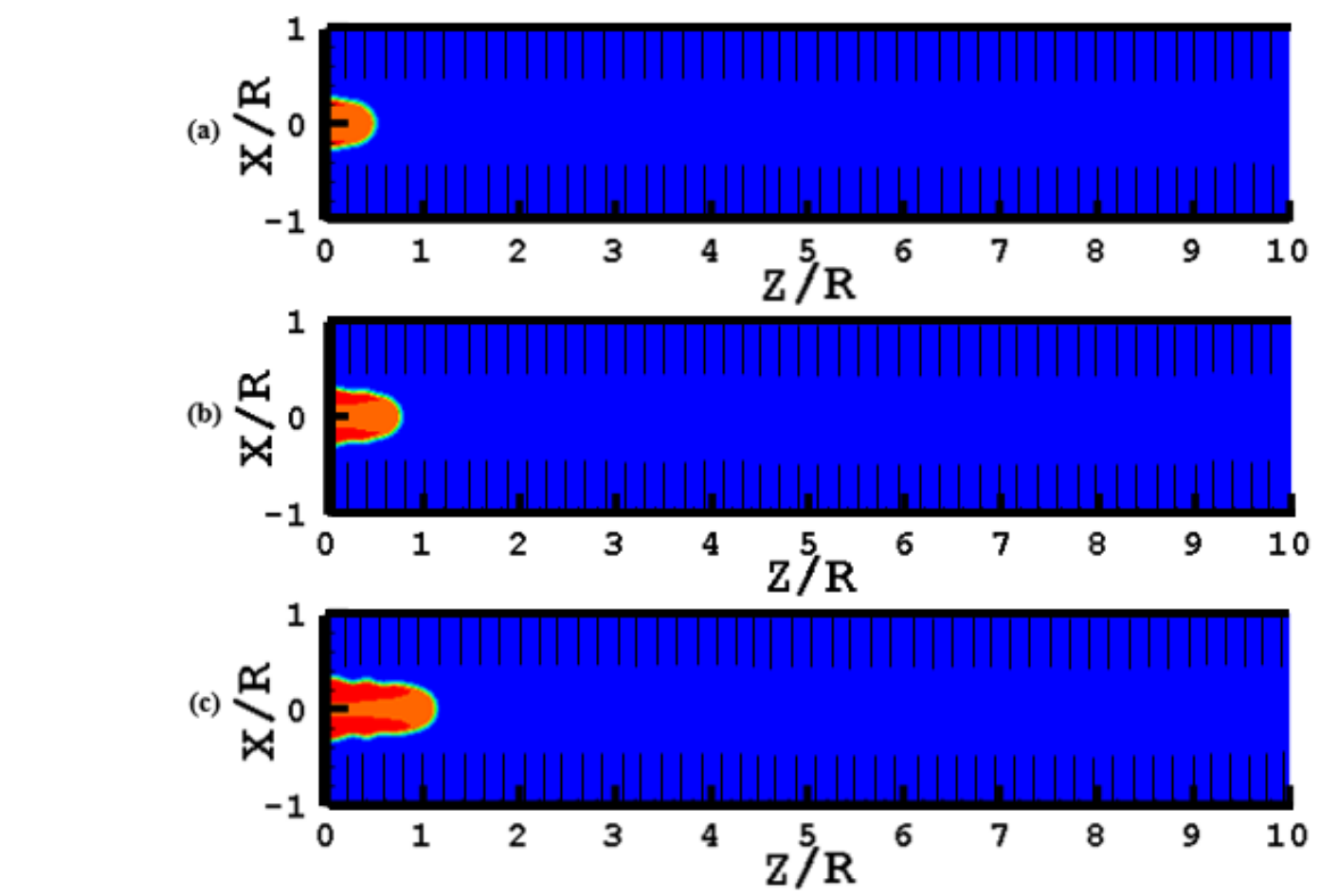

Figure 4.14-Temperature profiles for flames with $R e=48, \alpha=2 / 3$ and Le=2.0 for various scaled times $\tau=0.043(a) 0.063(b)$ $0.078(c)$

\subsection{Effect of Lewis number on the $\alpha$-dependence of flame acceleration}

Since the time of Ref. [7] it is known that the blockage ratio $\alpha$ is a key parameter influencing FA. However, it is recalled that Refs. [7-9] were limited to equidiffusive flames. Consequently, there is a fundamental interest to clarify if the $L e$-variations may influence the $\alpha$-dependence of FA and how. This question is addressed here. Figures 4.15-4.17 compare the role of blockage ratio at various $L e$ and $R e$. Specifically, Figures 4.15 (a-c) compare the scaled flame tip velocities for various $\alpha=1 / 3,1 / 2,2 / 3$ in each figure, with fixed $L e=0.2$ in Figure 4.15a, $L e=1.0$ in Figure $4.15 \mathrm{~b}$, and $L e=2.0$ in Figure 4.15c; with fixed $R e=24$ in all cases of Figure 4.15. Figures 4.16(a-c) constitute the complete counterparts of Figure 4.15(a-c), but for a wider channel, $R e=36$. Eventually, Figures 4.17(a-c) are the complete counterparts of Figures 4.15 and 4.16, but for even wider channel, $R e=48$. In this respect, Figures 4.15-4.17 actually reproduce the analysis of Figures 4.9-4.11, but for $\alpha$ instead of $L e$.

It is seen from Figures 4.15-4.17 that the $\alpha$-dependence of FA is significant. This is however not the original result but known from the previous studies [7-9]. A novel outcome here is a fact that $L e$-variations $d o$ modify the $\alpha$-dependence substantially. Well, the $\alpha$-dependence of FA does 
not change the sign due to $L e$, but there is a noticeably quantitative effect such that the $\alpha$ dependence is stronger for $L e<1$ combustion. At the same time, the difference in $\alpha$-dependence for $L e=1$ flames and $L e=2$ is relatively minor.

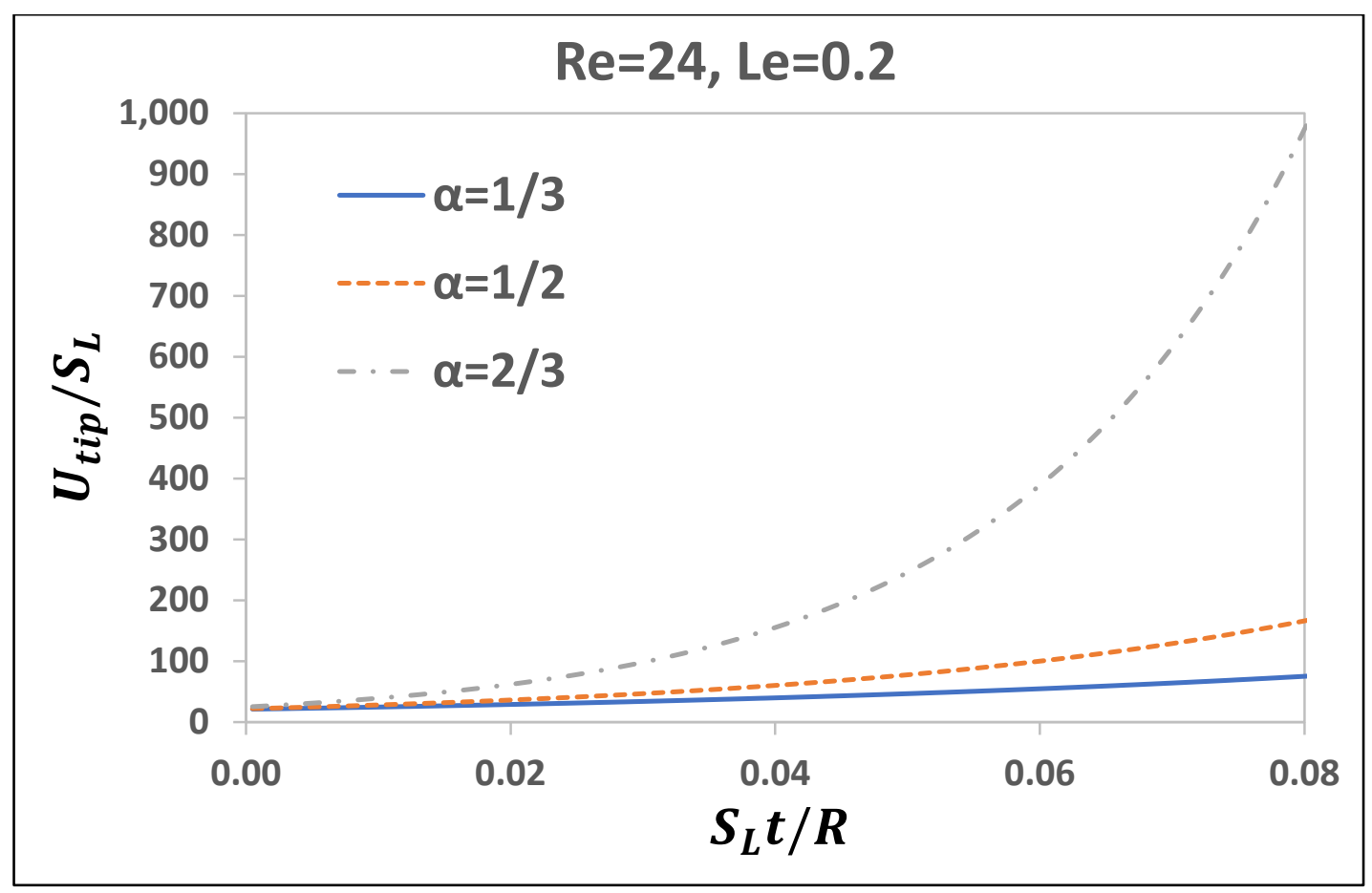

Figure 4.15a-Scaled flame tip velocity as a function of scaled time for fixed Re=24 and Le=0.2 


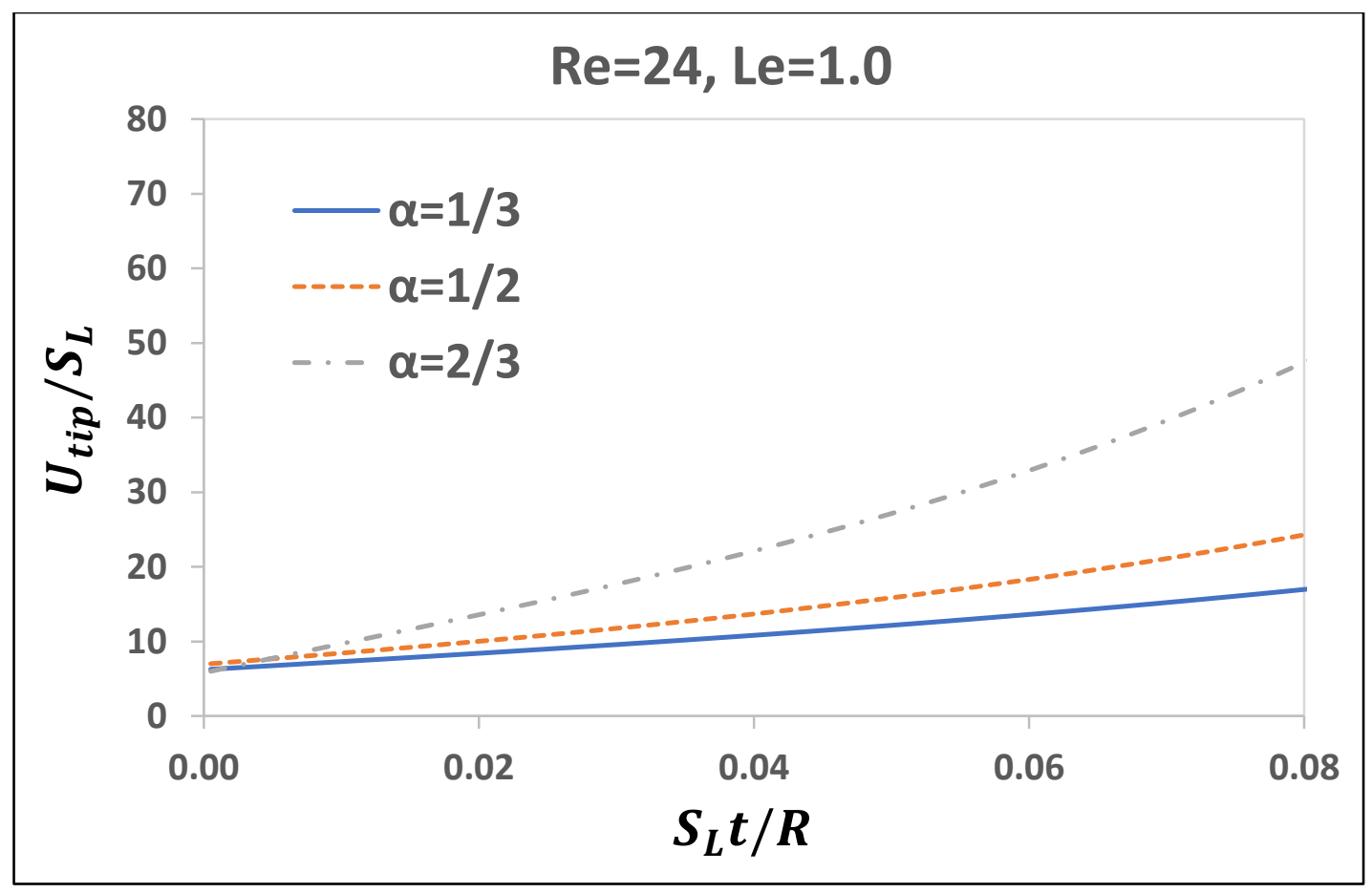

Figure 4.15b-Scaled flame tip velocity as a function of scaled time for fixed $R e=24$ and Le=1.0

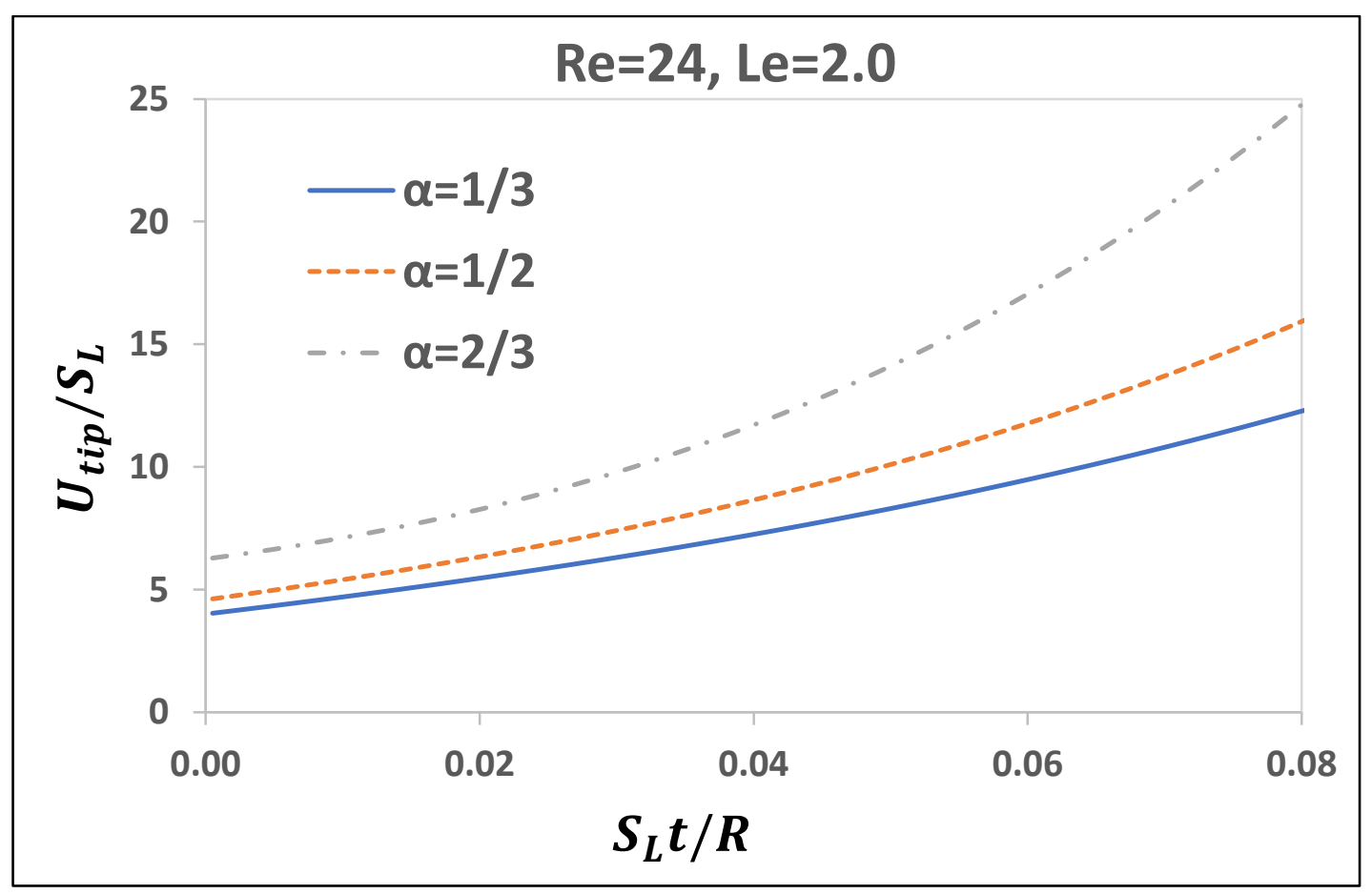

Figure $4.15 c$-Scaled flame tip velocity as a function of scaled time for fixed $R e=24$ and Le=2.0 


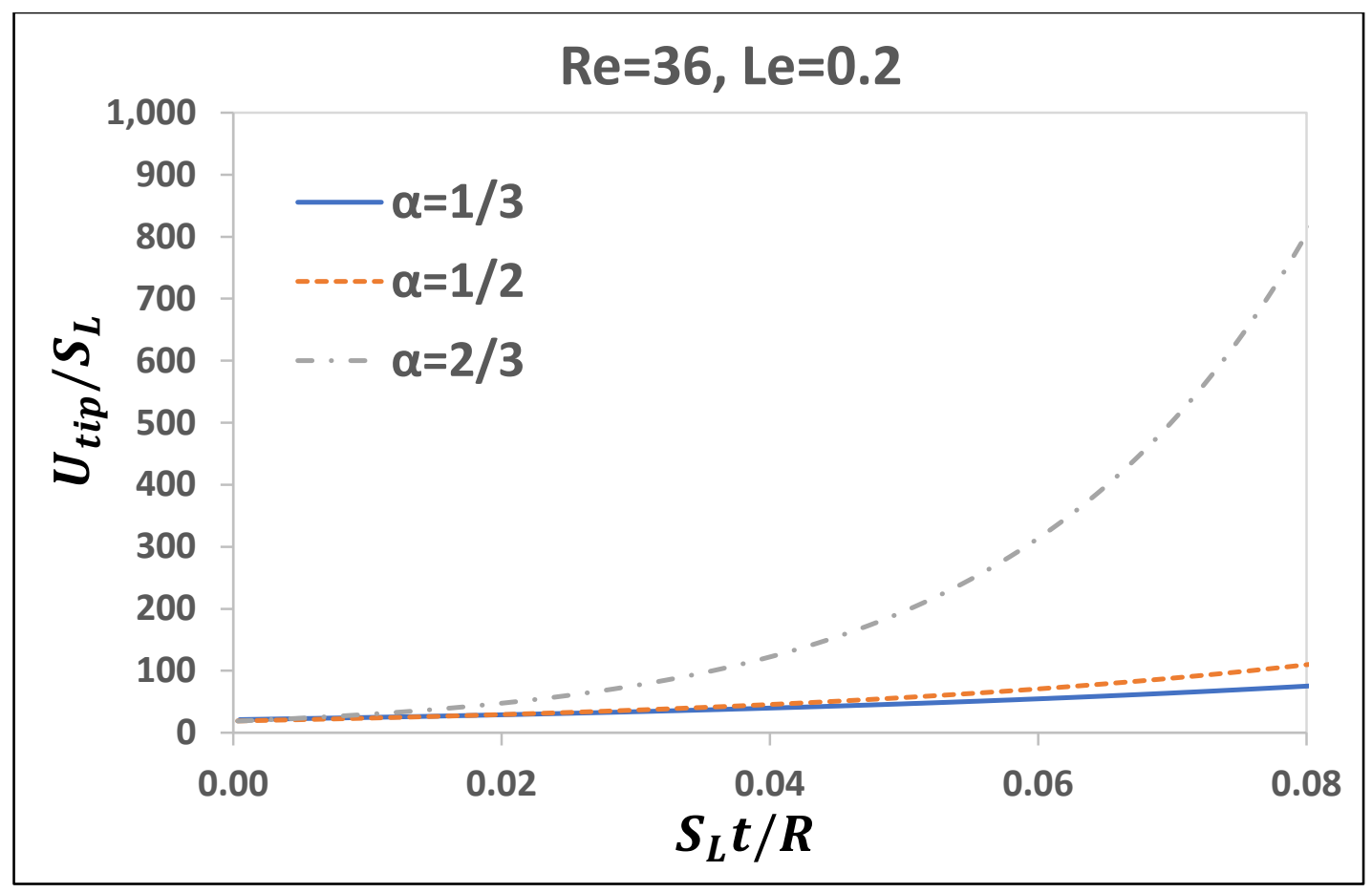

Figure 4.16a-Scaled flame tip velocity as a function of scaled time for fixed Re=36 and Le=0.2

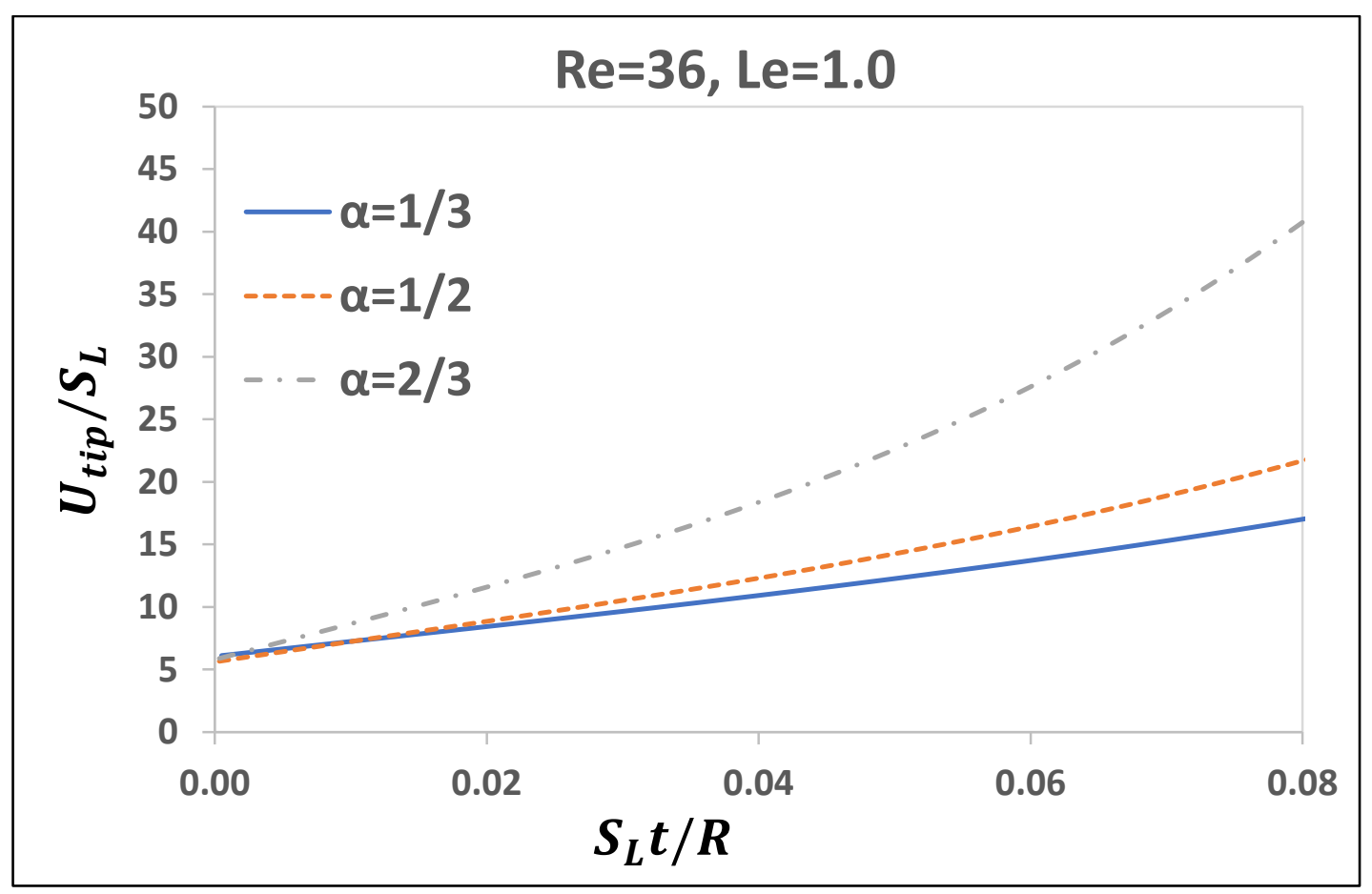

Figure 4.16b-Scaled flame tip velocity as a function of scaled time for fixed Re $=36$ and Le=1.0 


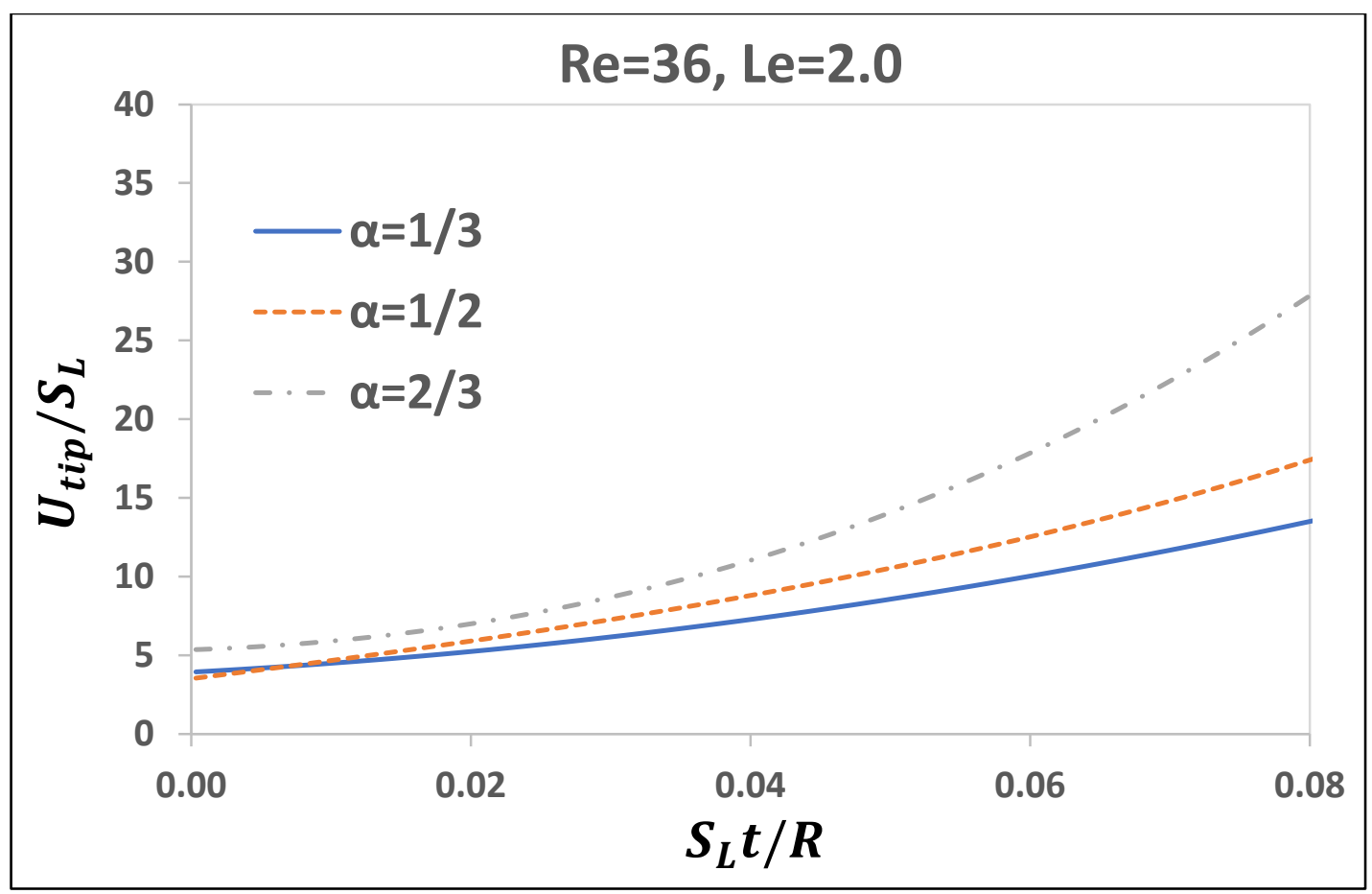

Figure 4.16c-Scaled flame tip velocity as a function of scaled time for fixed Re=36 and Le=2.0

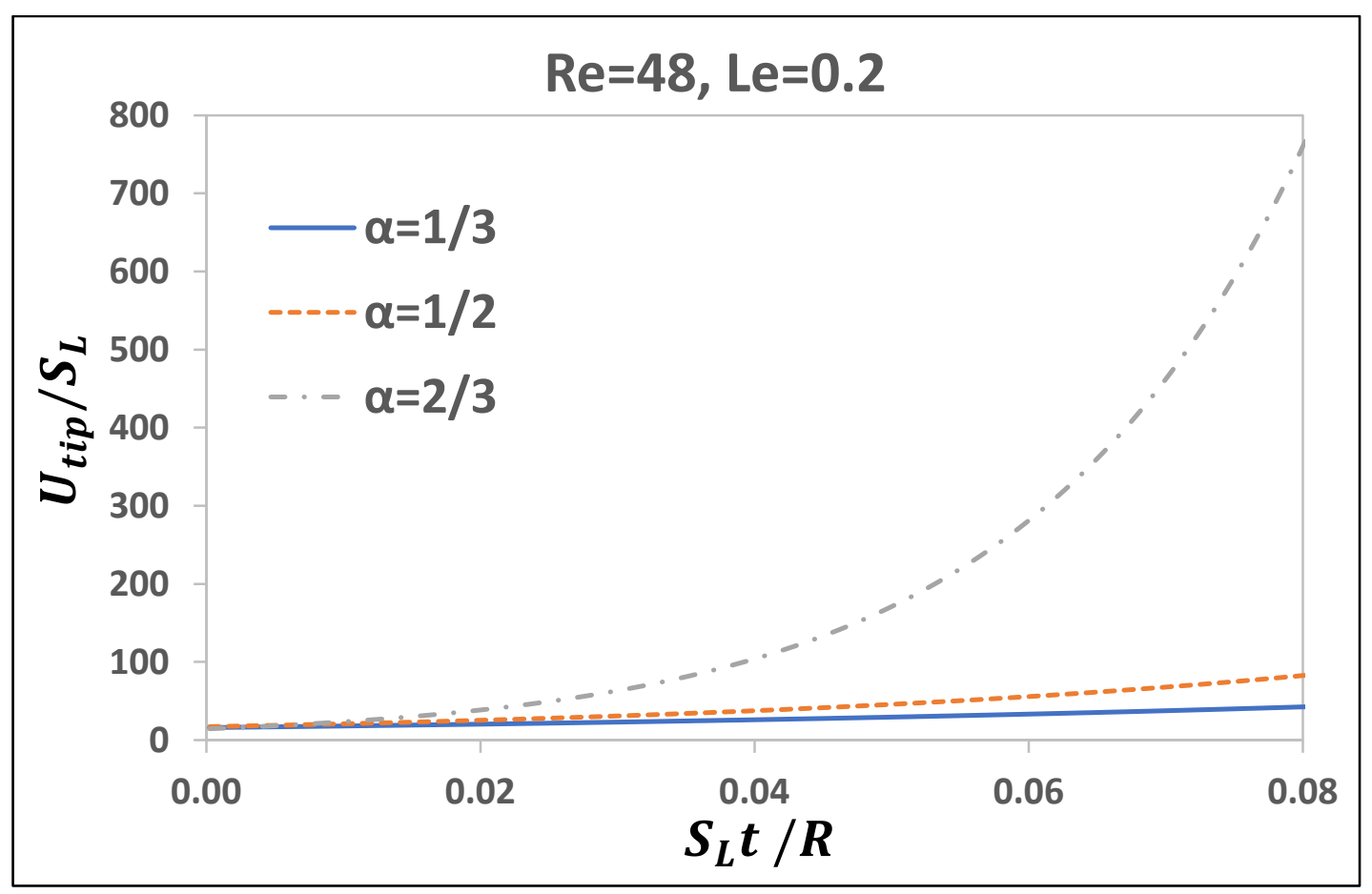

Figure 4.17 - Scaled flame tip velocity as a function of scaled time for fixed $R e=48$ and Le=0.2 


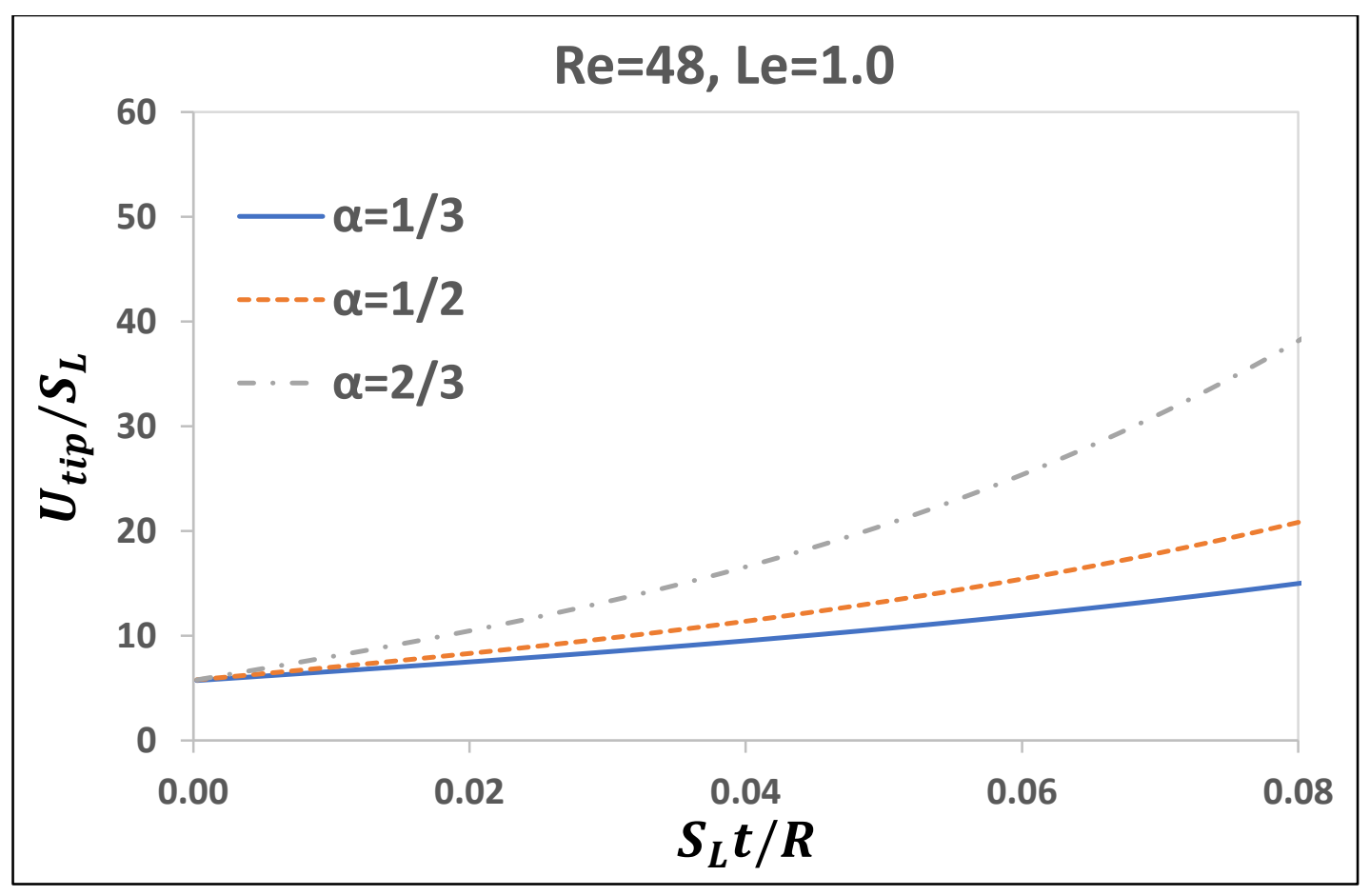

Figure 4.17b-Scaled flame tip velocity as a function of scaled time for fixed $R e=48$ and Le=1.0

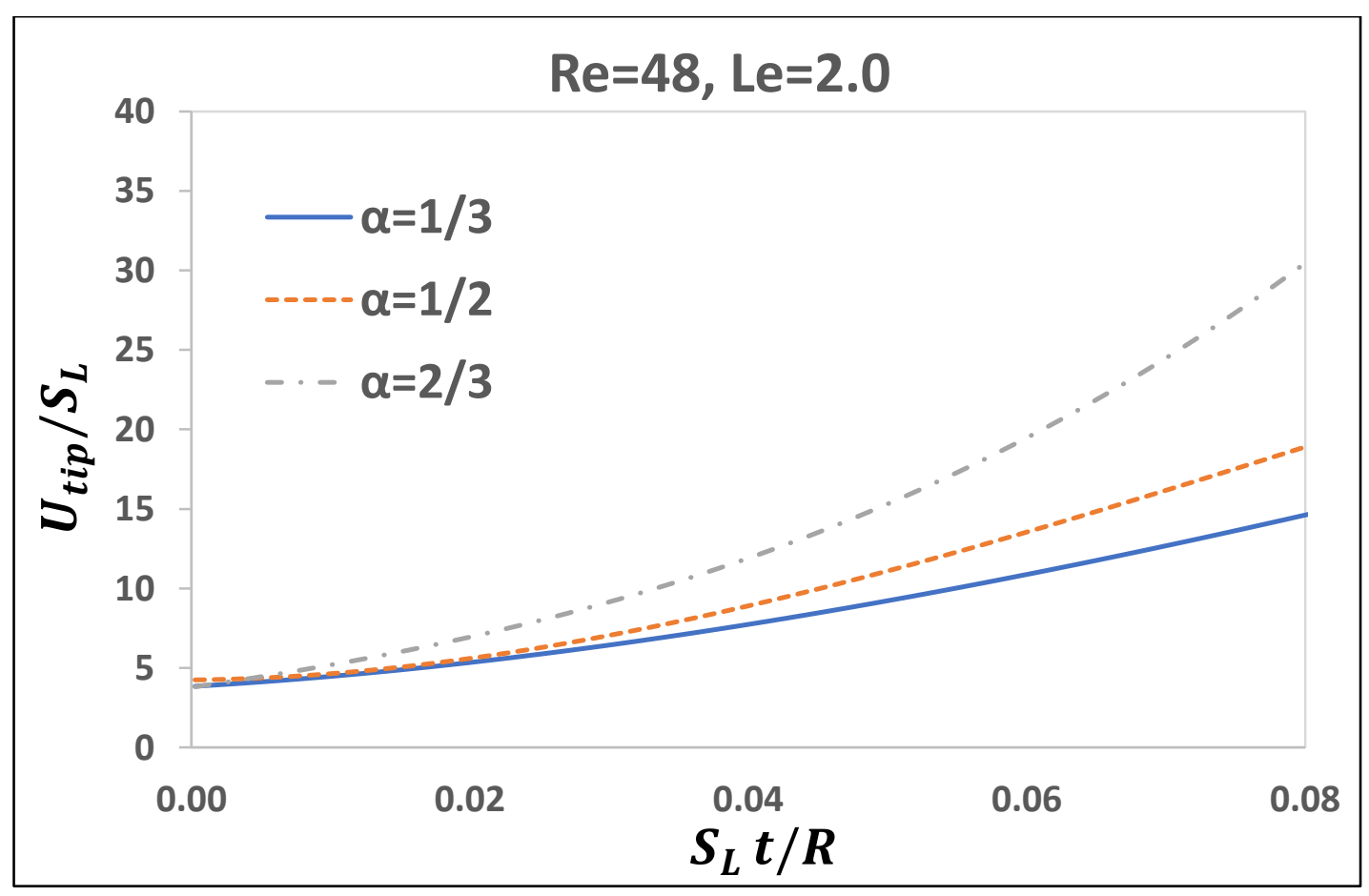

Figure 4.17 c-Scaled flame tip velocity as a function of scaled time for fixed $R e=48$ and Le=2.0 
Similar to the analysis performed by means of color snapshots at Figures $4.12-4.14$, it was also studied that the $\alpha$-dependence of the flame evolution with the help of color temperature snapshots in Figures 4.18-4.20. Here, in all three figures, the snapshots are taken at the scaled time instants $\tau=0.046(a), 0.066(b), 0.079(c)-$ for $\alpha=1 / 3$ in Figure 4.18, $\alpha=1 / 2$ in Figure 4.19, and $\alpha=2 / 3$ in Figure 4.20. In all these cases of Figures 4.18-4.20, the channel of width $R=36$ and the same equidiffusive flame $(L e=1)$ was employed. Obviously, the snapshots of Figure 4.20, which is associated with the largest blockage ratio, $\alpha=2 / 3$, shows the fastest flame evolution. Nevertheless, it is noted that the results are not as significant as that of varied Le number, which is in line with the statement that the Lewis effect is stronger than that of the blockage ratio.

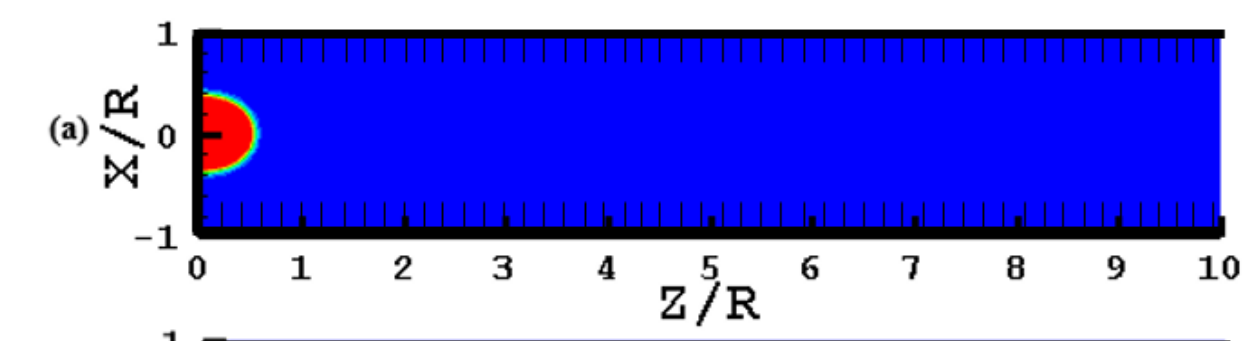

(b)

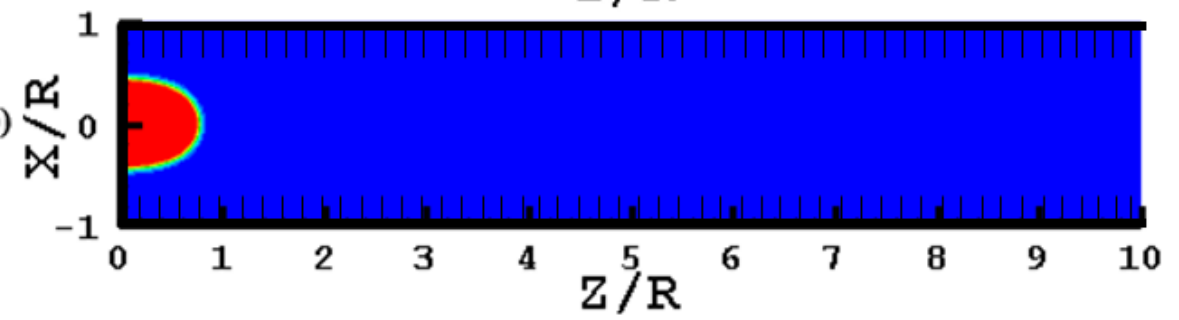

(c)

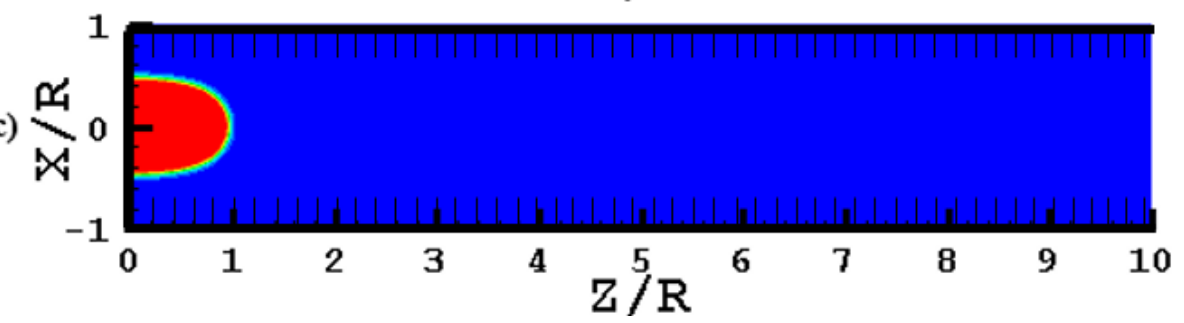

Figure 4.18 - Temperature profile of flames with $R e=36, L e=1.0$ and $\alpha=1 / 3$ for various scaled times $\tau=0.046(a)$, 0.066(b), $0.079(c)$ 

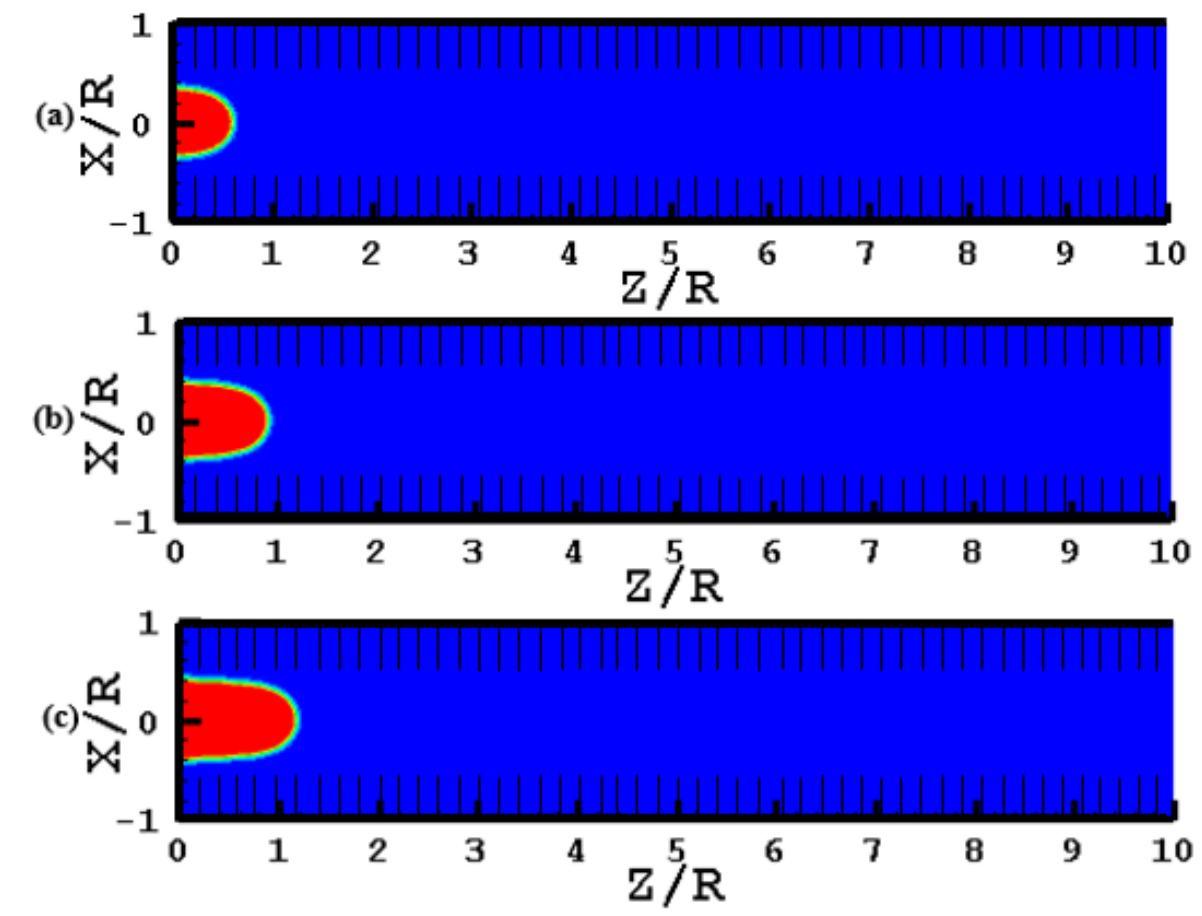

Figure 4.19 - Temperature profile of flames with $R e=36, L e=1.0$ and $\alpha=1 / 2$ for various scaled times $\tau=0.046(a), 0.066(b)$, $0.079(c)$
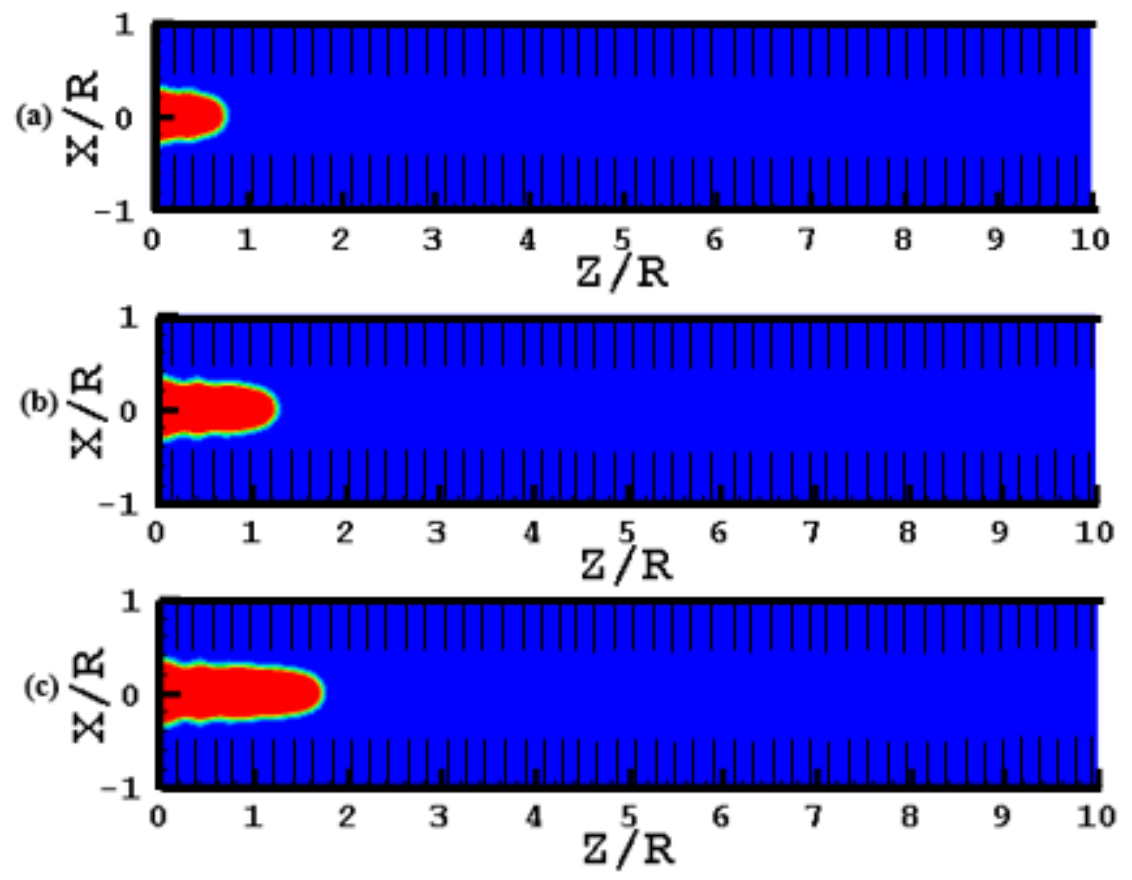

Figure 4.20 - Temperature profile of flames with $R e=36, L e=1.0$ and $\alpha=2 / 3$ for various scaled times $\tau=0.046(a), 0.066(b)$, $0.079(c)$ 


\subsection{The effect of Lewis number on the Re-dependence of flame acceleration}

Finally, while the theory [7] yields that the Bychkov acceleration mechanism is Re-independent, some Re-dependence may actually occur in the practical reality. Moreover, Le-variations may potentially impact such a Re-dependence. This question is also addressed in the present work by means of the plots in Figures 4.21-4.23 and the color snapshots of the flame evolution in Figures 4.24-4.26. Specifically, Figures 4.21(a-c) compare the scaled flame tip velocities for various $R e=24,36,48$ in each figure, with fixed $L e=0.2$ in Figure 4.21a, $L e=1$ in Figure 4.21b, and $L e=2$ in Figure 4.21c; with fixed $\alpha=1 / 3$ in all cases of Figure 4.21. Figures 4.22(a-c) are the counterparts of Figure 4.21(a-c), but for a larger blockage ratio, $\alpha=1 / 2$. Eventually, Figures 4.23(a-c) are the complete counterparts of Figures 4.21 and 4.22, but for largest blockage ratio considered, $\alpha=2 / 3$.

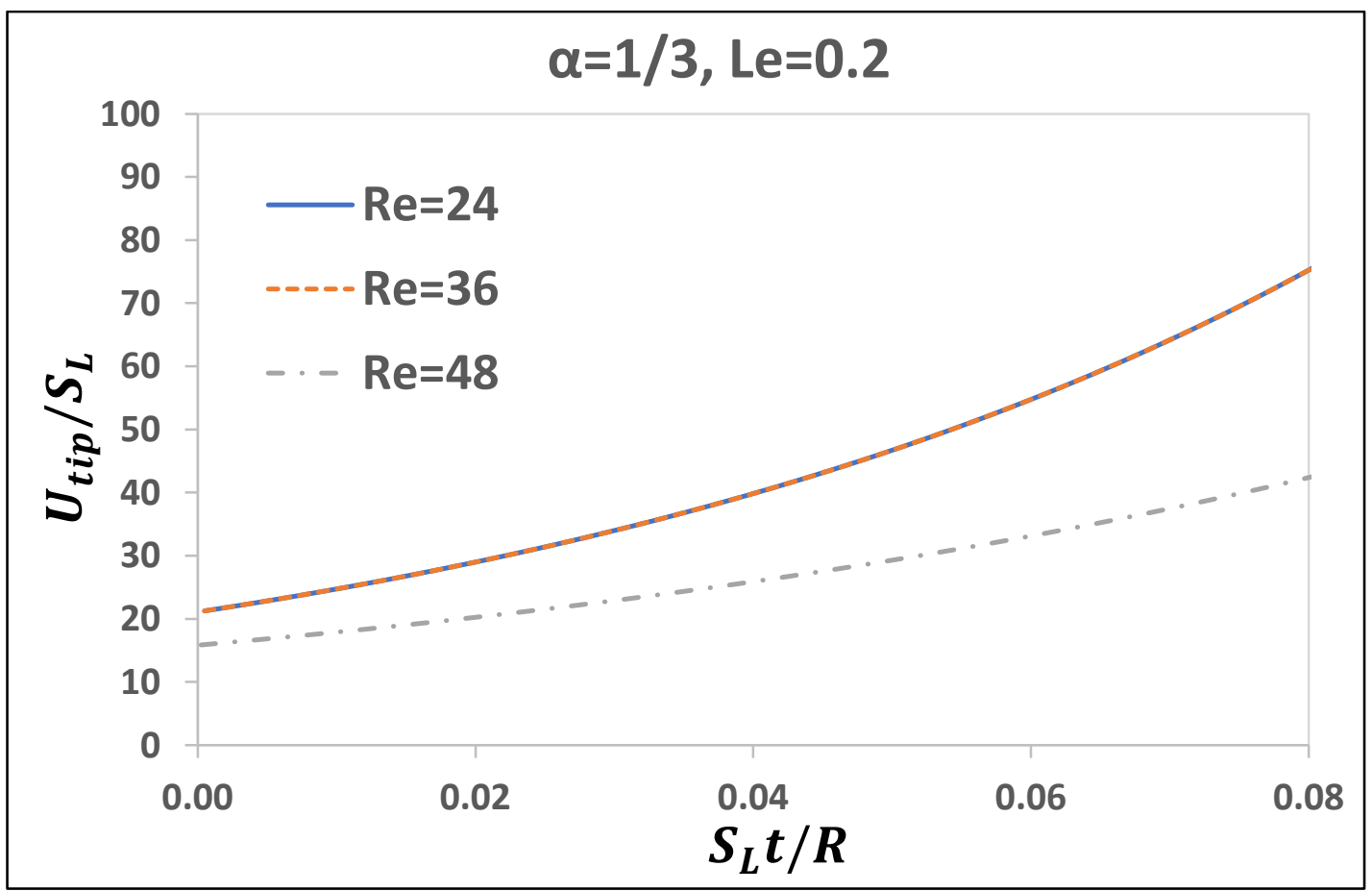

Figure 4.21a-Scaled flame tip velocity as a function of scaled time for fixed $\alpha=1 / 3$ and Le=0.2 


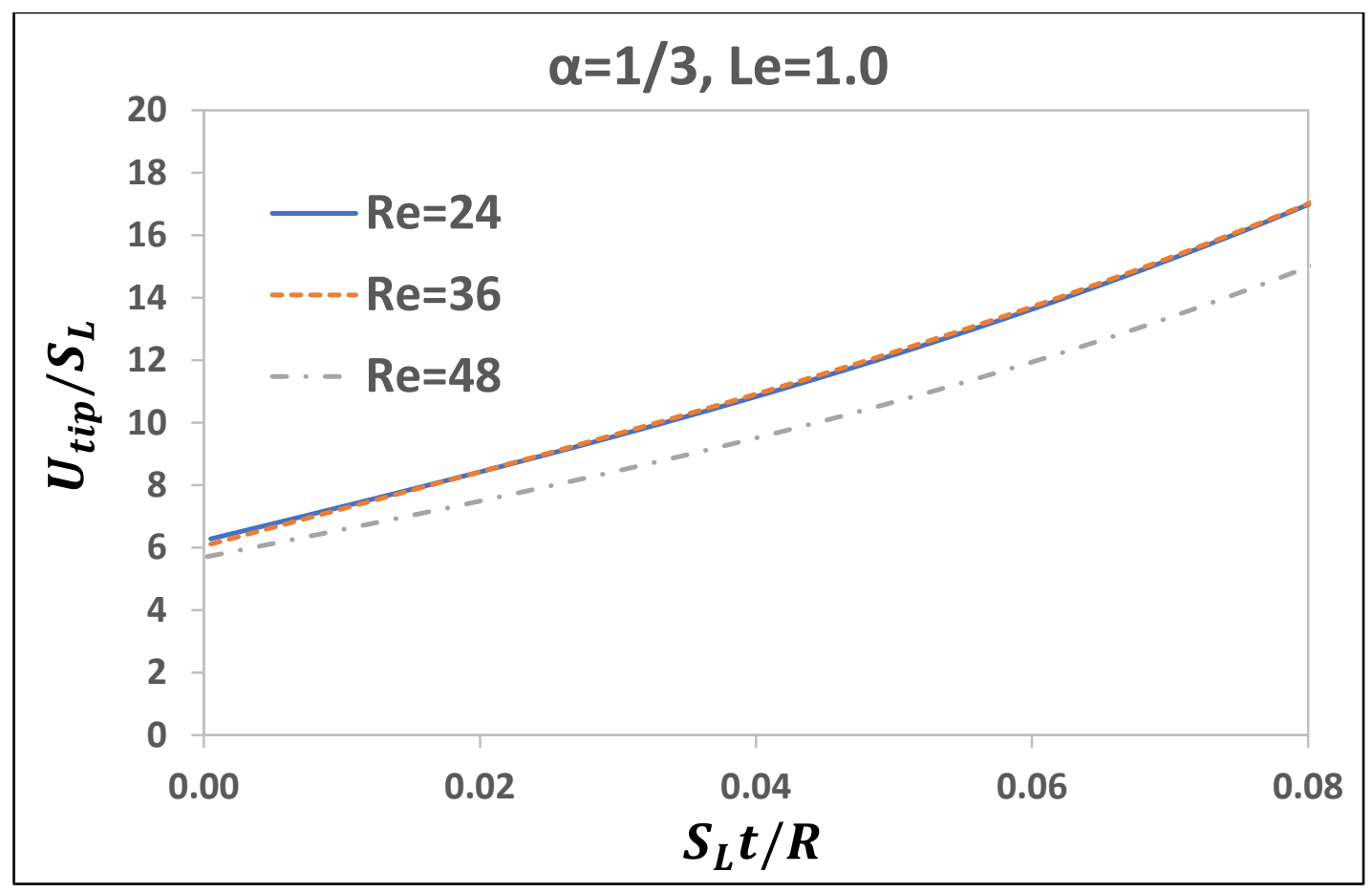

Figure 4.21b - Scaled flame tip velocity as a function of scaled time for fixed $\alpha=1 / 3$ and Le=1.0

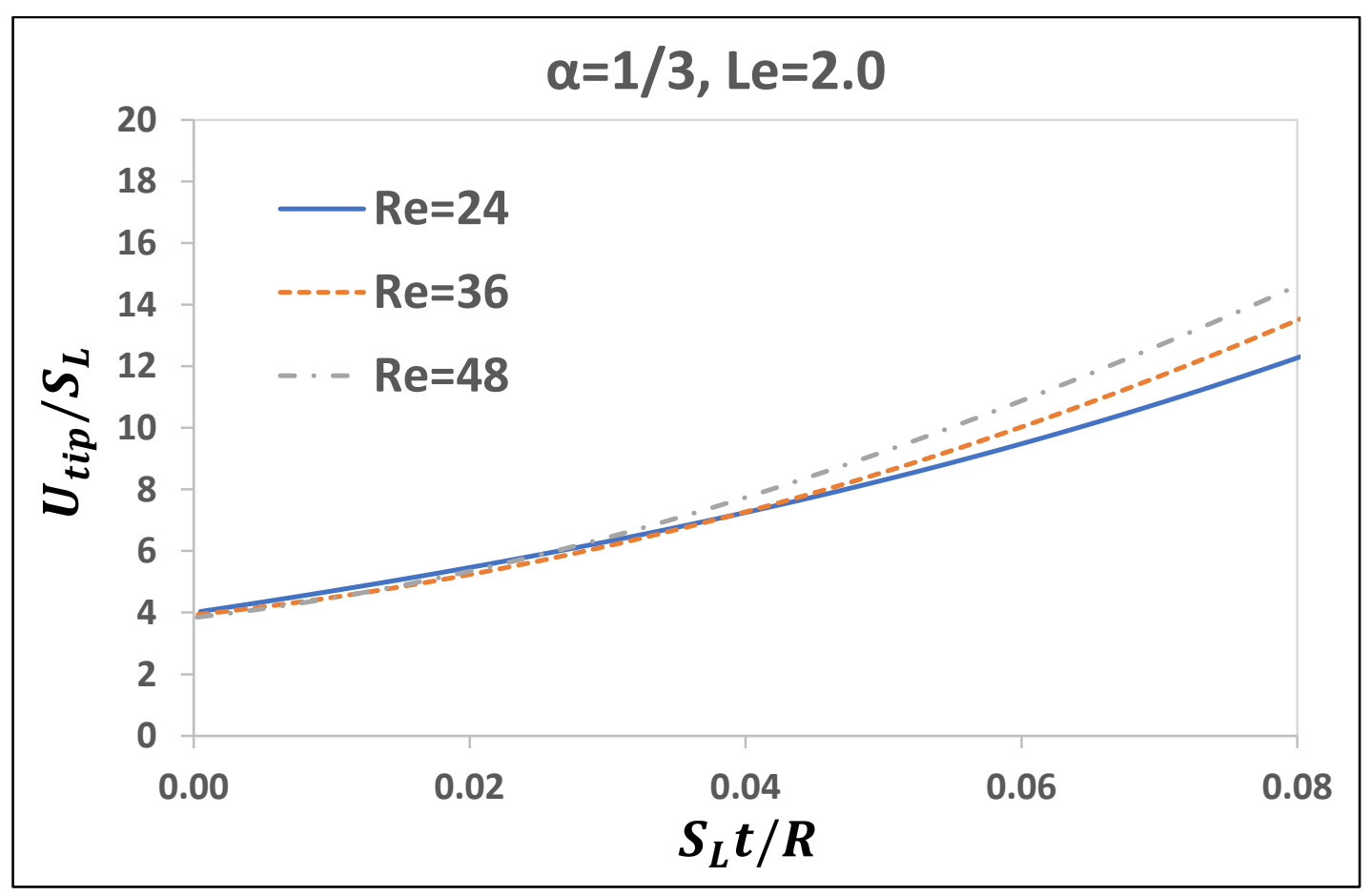

Figure 4.21c-Scaled flame tip velocity as a function of scaled time for fixed $\alpha=1 / 3$ and Le=2.0 


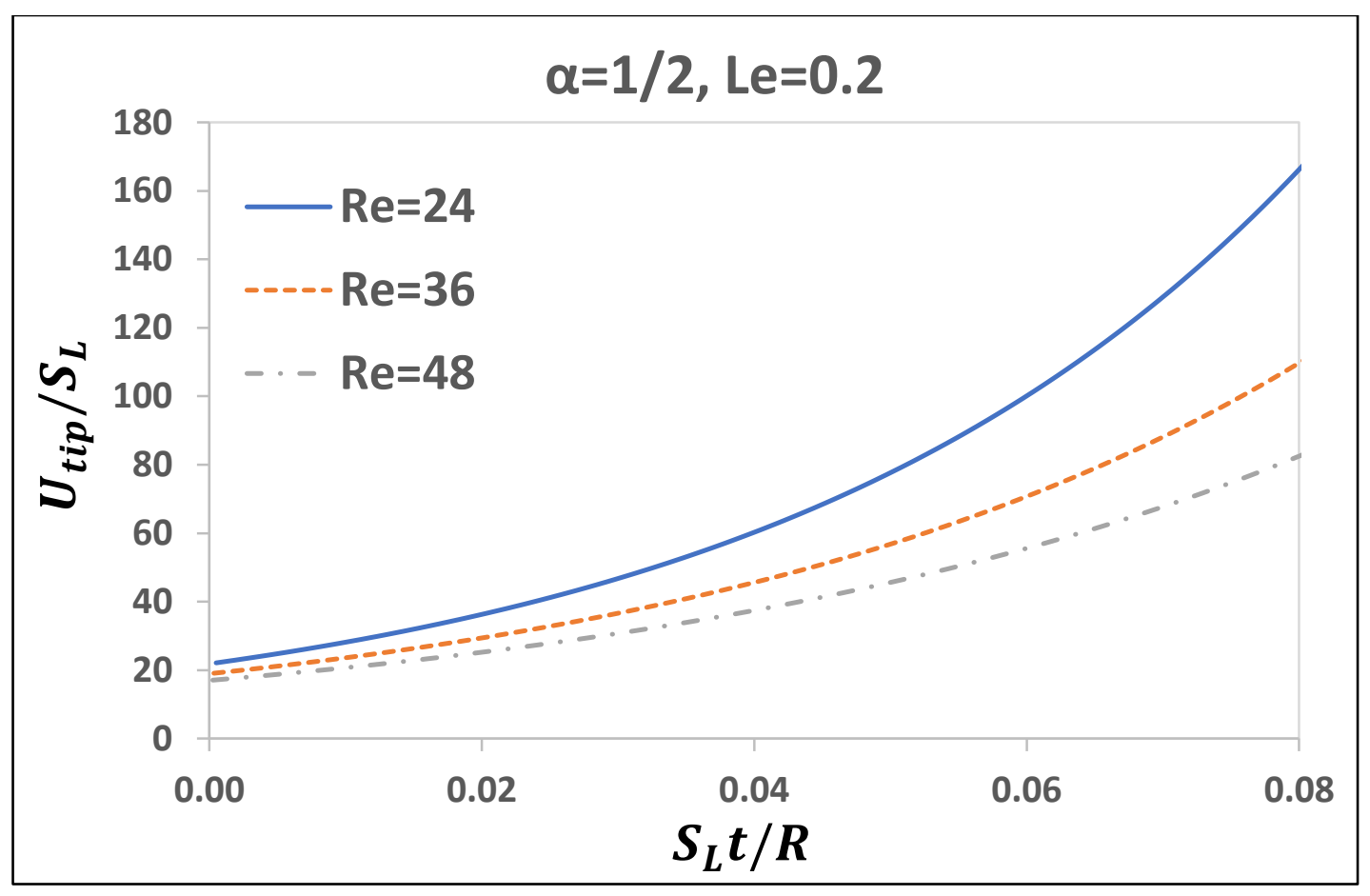

Figure 4.22a - Scaled flame tip velocity as a function of scaled time for fixed $\alpha=1 / 2$ and Le=0.2

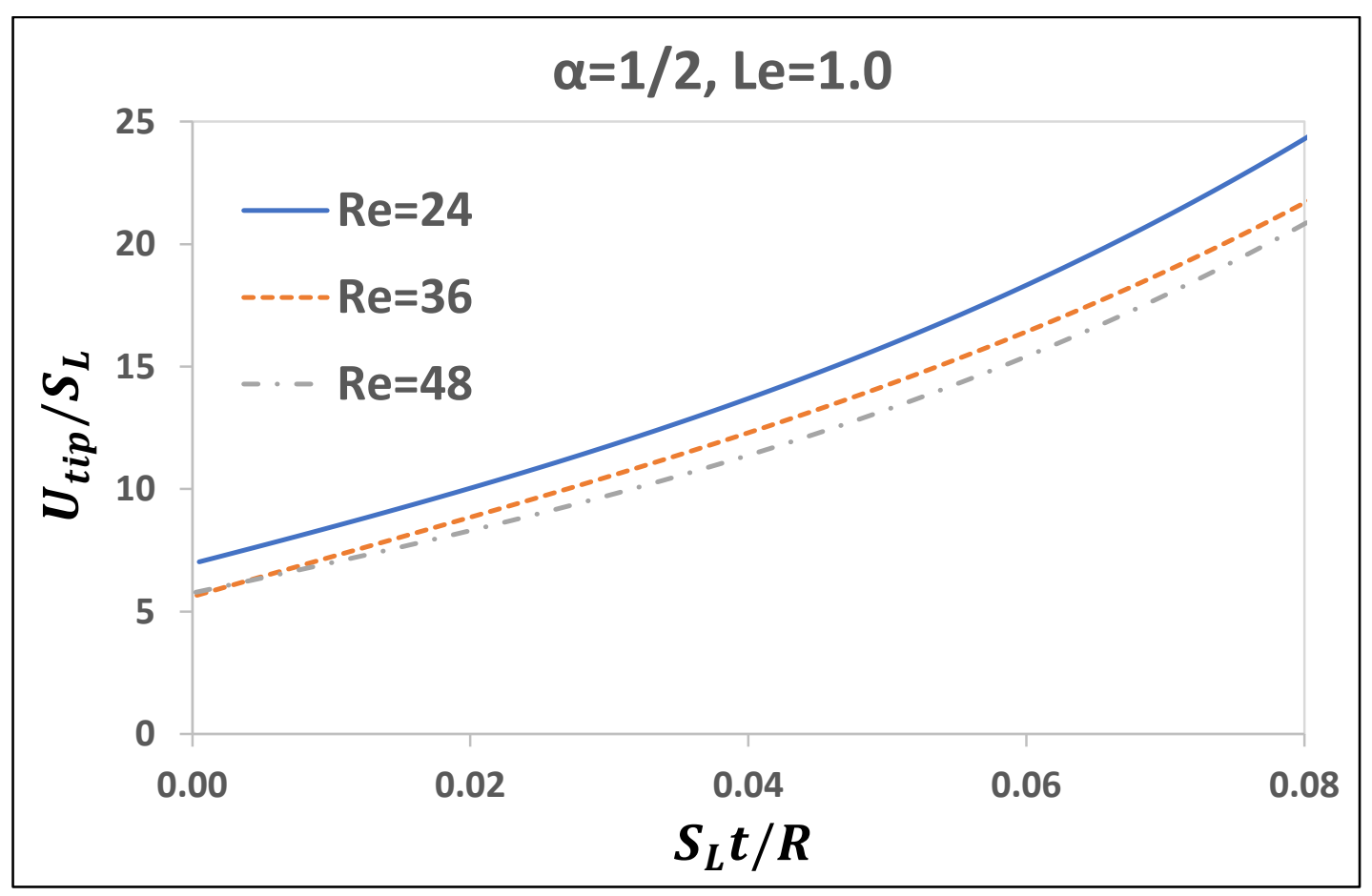

Figure $4.22 b$ - Scaled flame tip velocity as a function of scaled time for fixed $\alpha=1 / 2$ and Le=1.0 


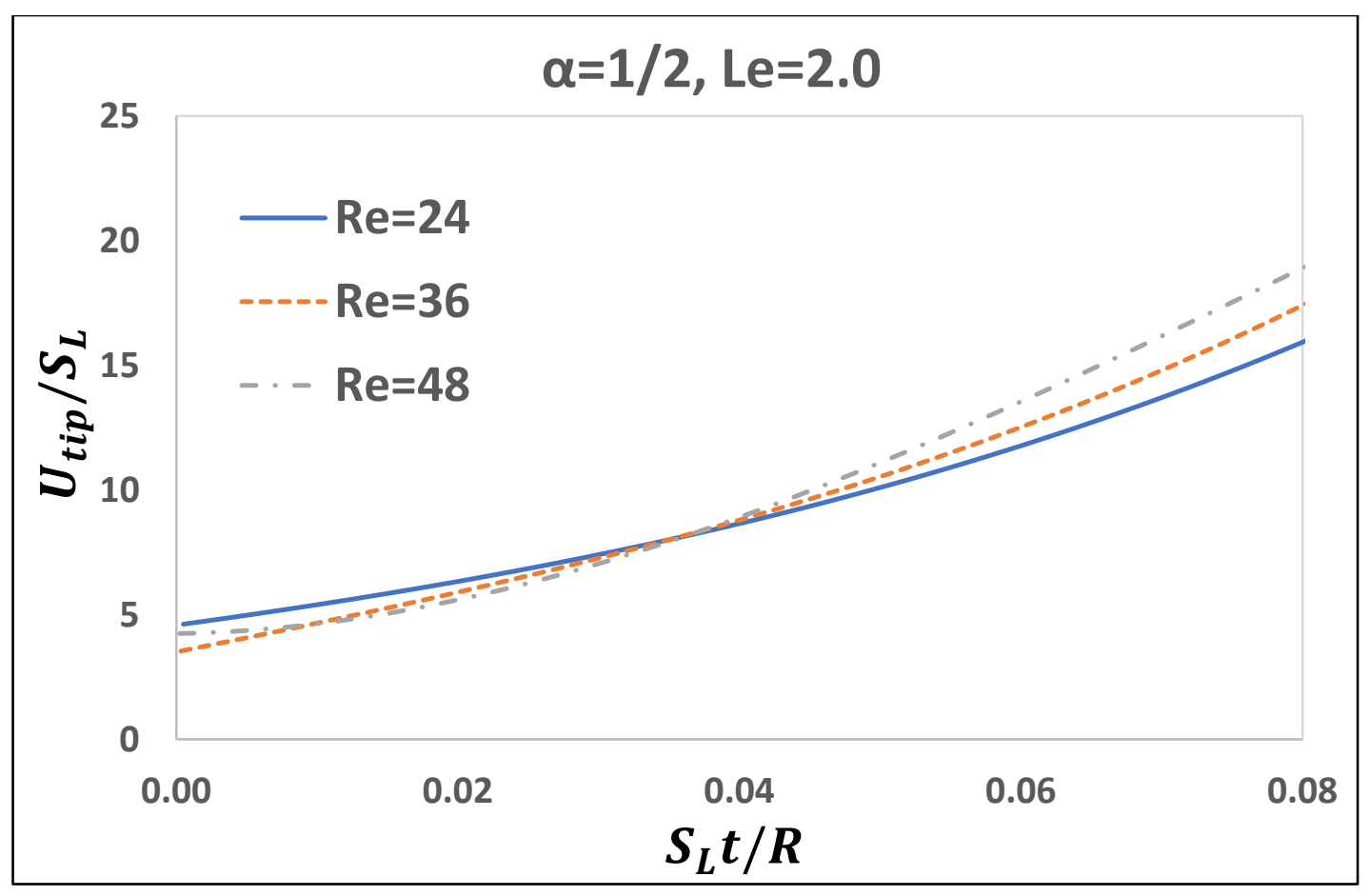

Figure 4.22c-Scaled flame tip velocity as a function of scaled time for fixed $\alpha=1 / 2$ and Le=2.0

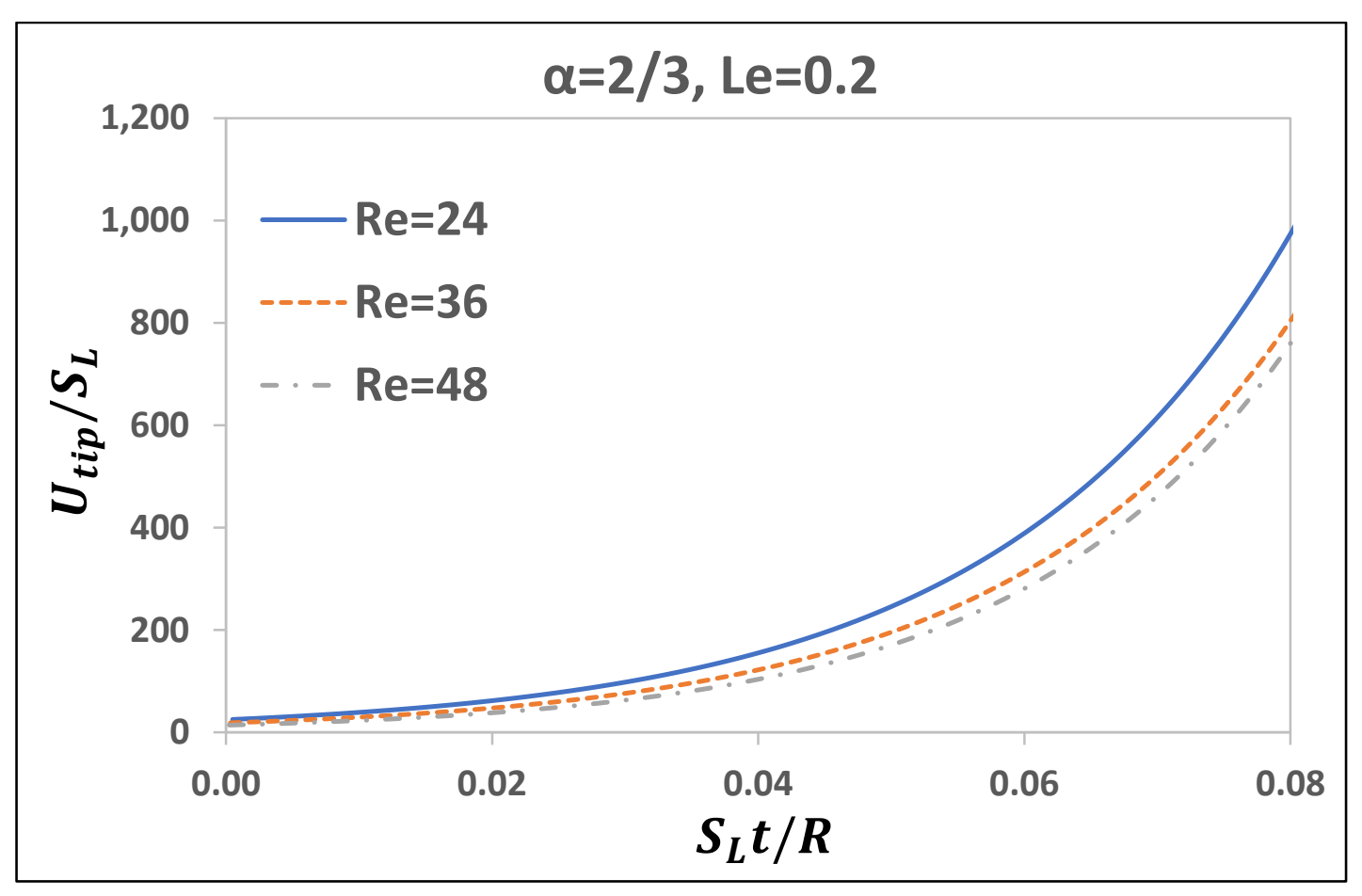

Figure $4.23 a$-Scaled flame tip velocity as a function of scaled time for fixed $\alpha=2 / 3$ and Le=0.2 


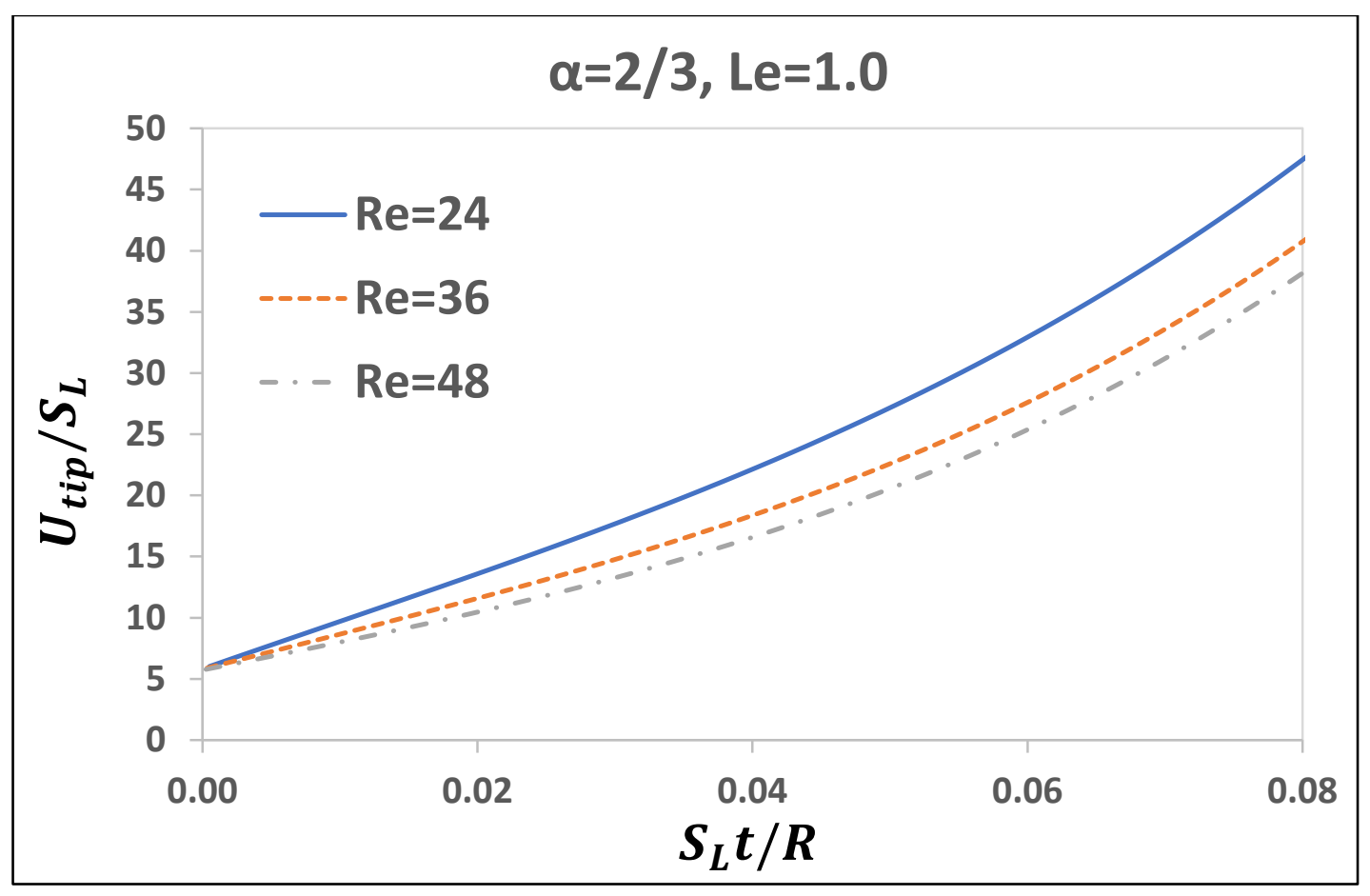

Figure $4.23 b$ - Scaled flame tip velocity as a function of scaled time for fixed $\alpha=2 / 3$ and Le=1.0

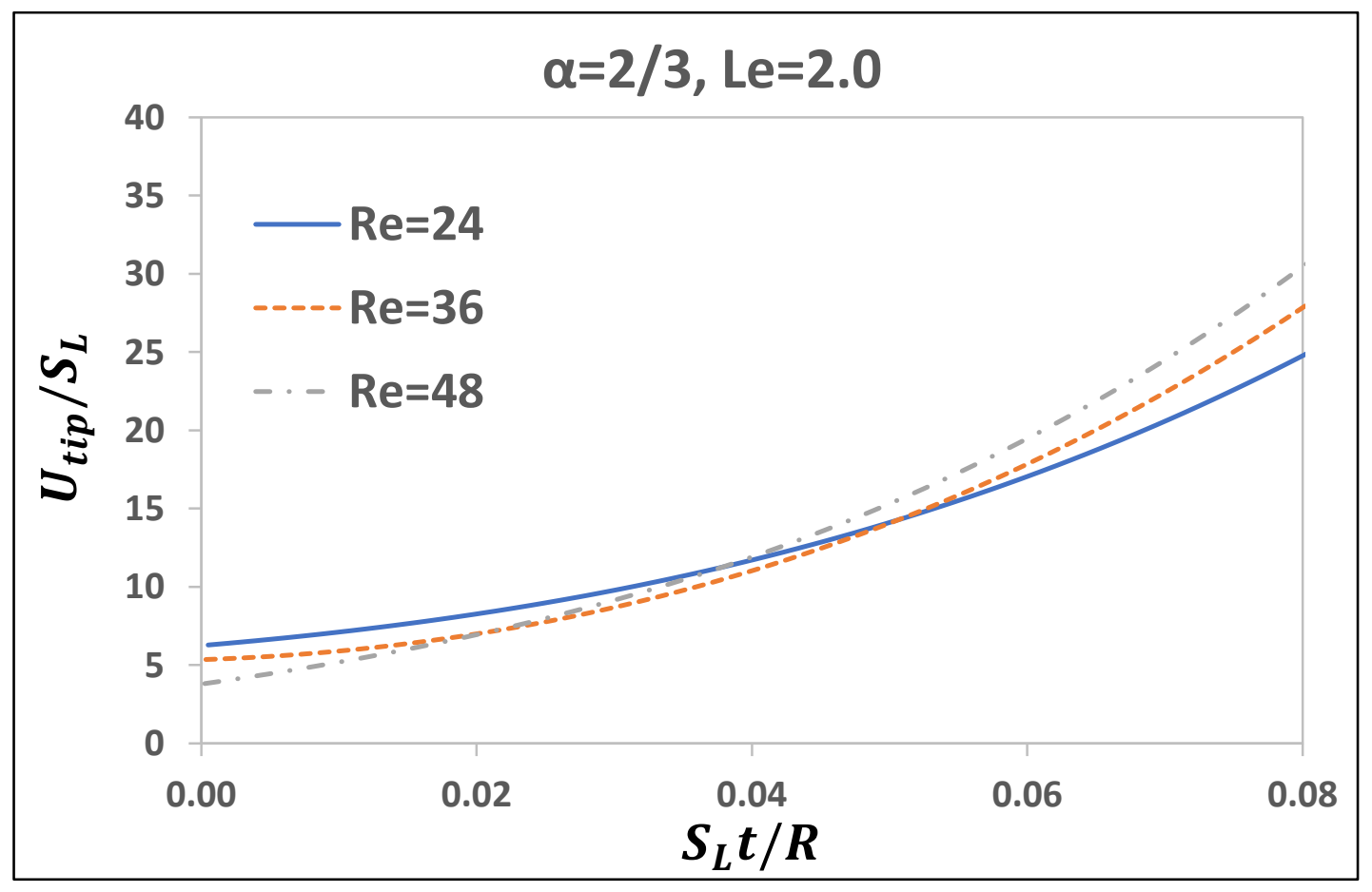

Figure $4.23 c$-Scaled flame tip velocity as a function of scaled time for fixed $\alpha=2 / 3$ and Le=2.0 
It is seen that the impact of Re is actually minor as all the curves for $R e=24,36,48$ go close in all nine cases. This supports the Bychkov formulation [7] predicting Re-independent FA, see Eqs. (2.1), (2.2), and (2.5). On the other hand, Figures 4.21c, 4.22c and 4.23c (for $L e=2$ ) show a very intriguing result: though the Re-dependence is quite weak, the impact of Le may change it, up to the opposite trend. Indeed, while FA weakens with $R e$ for the $L e \leq 1$ flames, Figures 4.21(a,b), 4.22(a,b), and $4.23(\mathrm{a}, \mathrm{b})$; it however gets promoted with $R e$ in the $L e>1$ case, see Figures $4.21 \mathrm{c}, 4.22 \mathrm{c}$ and $4.23 \mathrm{c}$. In this light, we can potentially look for a certain threshold $L e$ that would correspond to the change of the trend and thus provide the complete $R e$ independence.

A similar result about the impact of the flame Reynolds number on the flame propagation in obstructed pipes came from the snapshots of Figures 4.24-4.26. Here, in all these figures, the snapshots are taken at the scaled time instants $\tau=0.046(a), 0.062(b), 0.078(c)-$ for $R e=24$ in Figure 4.24, $R e=36$ in Figure 4.25, and $R e=48$ in Figure 4.26. In all these cases of Figures 4.24-4.26, I employed the same blockage ratio, $\alpha=1 / 3$, and the same nonequidiffusive flame, $L e=0.2$. It is seen that an increase in $R e$ moderates FA. When $R e=24$, Figure 4.24, the flame has propagated through about $3 \mathrm{R}$, with only $2 \mathrm{R}$ for $R e=48$, Figure 4.26 - for the same scaled time instant.

(a)

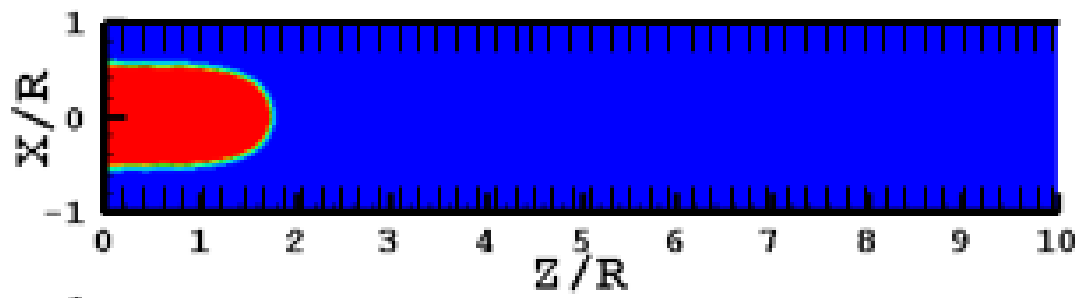

(b)
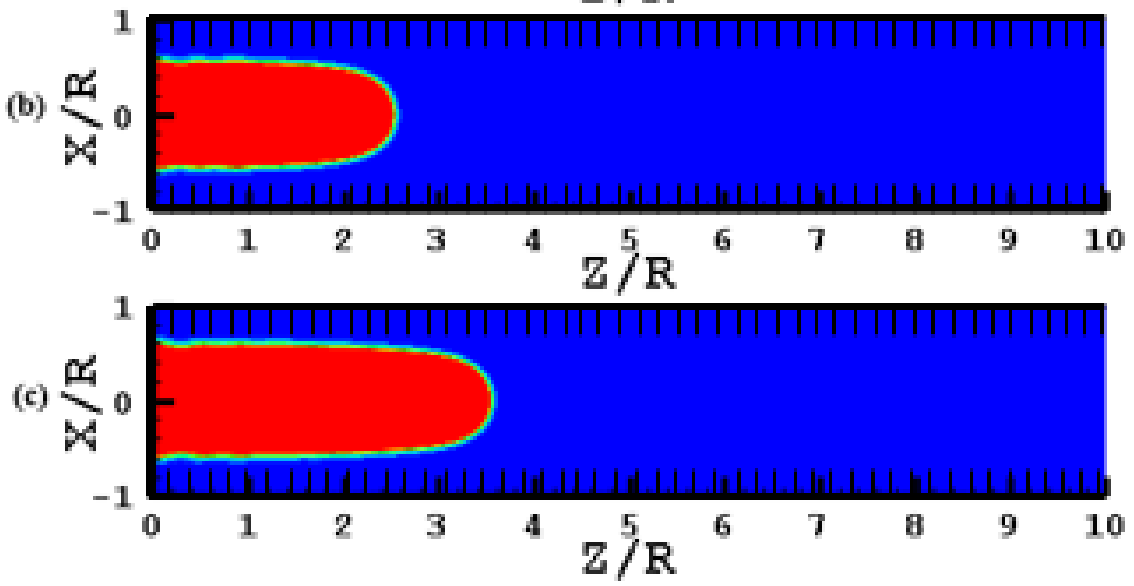

Figure 4.24 - Temperature profile of flame with Le=0.2, $\alpha=1 / 3$ and Re=24, for various scaled times $\tau=0.046(a) 0.062(b)$ $0.078(c)$ 
(a)

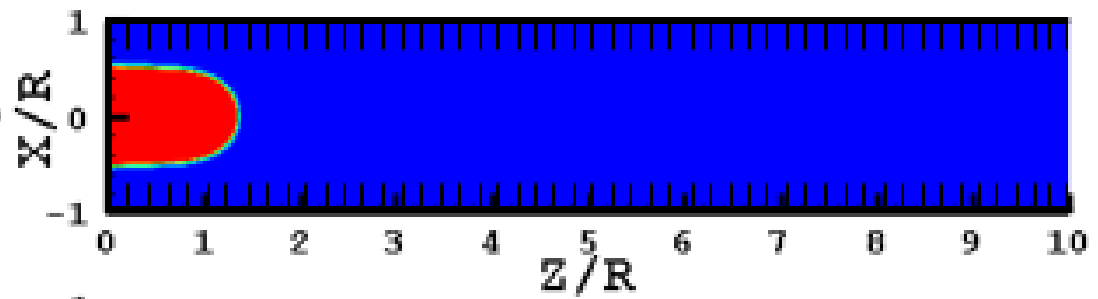

(b)

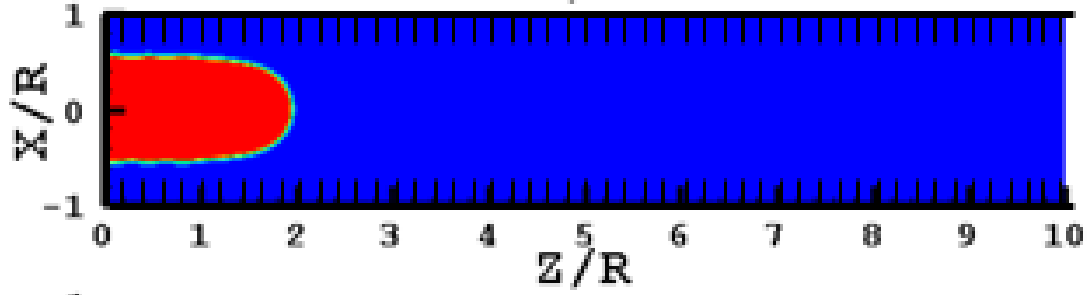

(c)

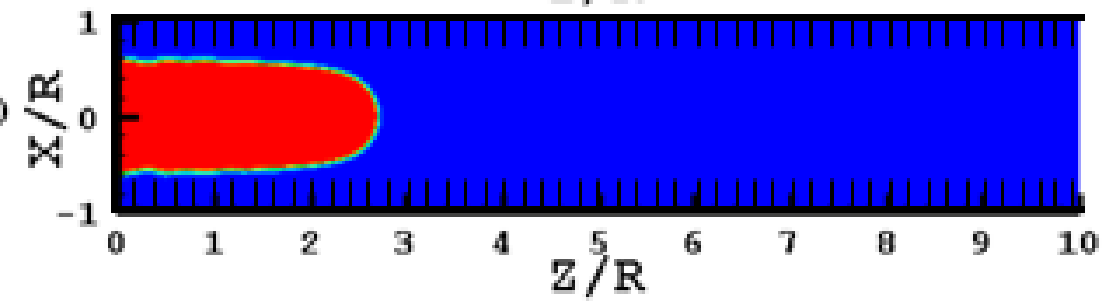

Figure 4.25 - Temperature profile of flame with $L e=0.2, \alpha=1 / 3$ and Re=36, for various scaled times $\tau=0.046(a) 0.062(b)$ $0.078(c)$
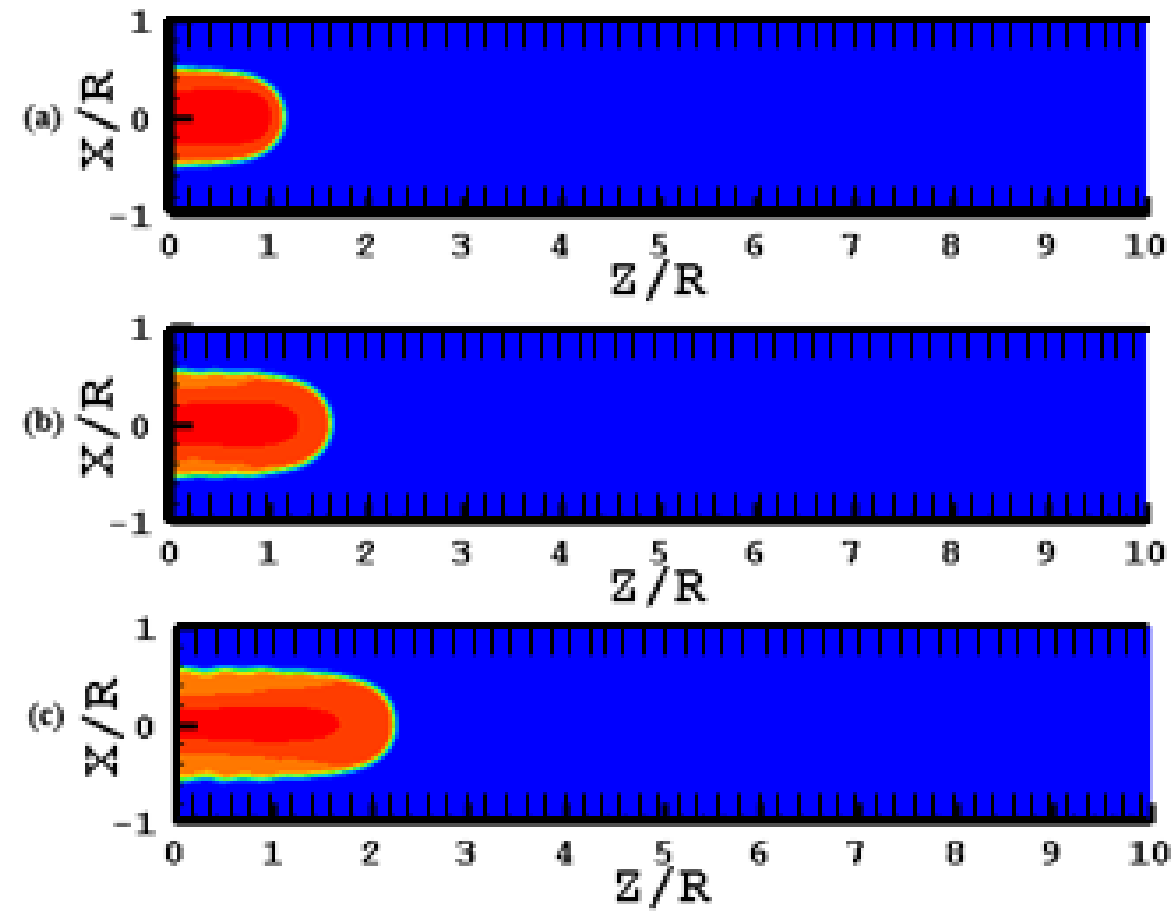

Figure 4.26 - Temperature profile of flame with $L e=0.2, \alpha=1 / 3$ and $R e=48$, for various scaled times $\tau=0.046(a) 0.062(b)$ $0.078(c)$ 


\subsection{Exponential Acceleration Rate}

Finally, I have investigated all the acceleration trends observed, and when acceleration exhibited an exponential trend, then the exponential acceleration rate was calculated. The result is plotted versus $L e$ in Figure 4.27 - for $R e=24,36,48$ in Figures 4.27(a-c), respectively, and for various $\alpha=1 / 3,1 / 2,2 / 3$ in each figure. As expected, the acceleration rate $\sigma$ appeared largest for the non-equidiffusive cases of $L e<1$. More specifically, the exponential acceleration trend was seen for $L e=0.2$ only for the low $\alpha$ cases i.e only $\alpha=1 / 3,1 / 2$. The absence of this trend in the $\alpha=2 / 3$ case is attributed to a strong competition between the $L e$ and $\alpha$ effect in that scenario.

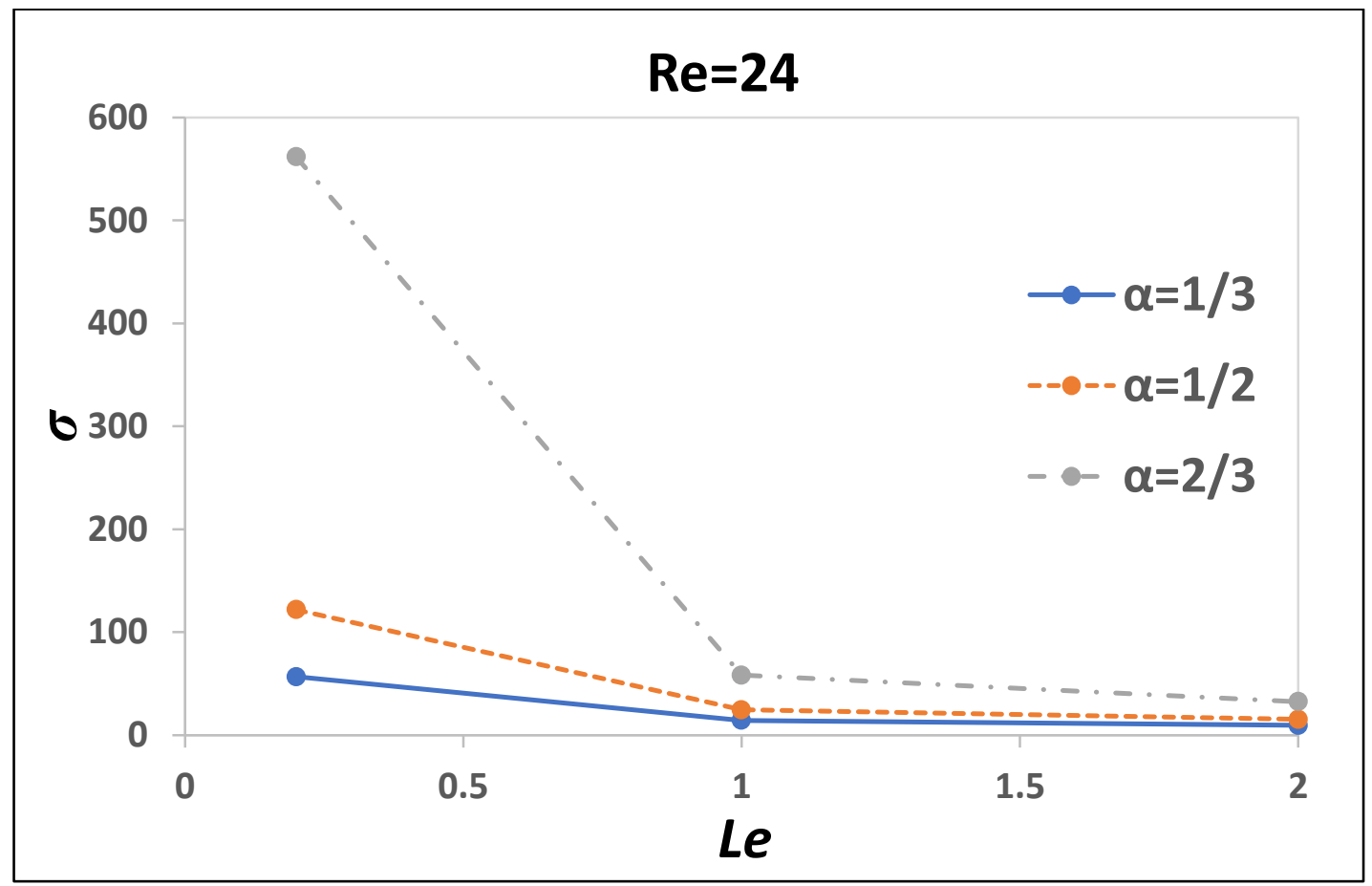

Figure $4.27 a$ - The exponential acceleration rate $v$ s the Lewis number for fixed $R e=24$ 


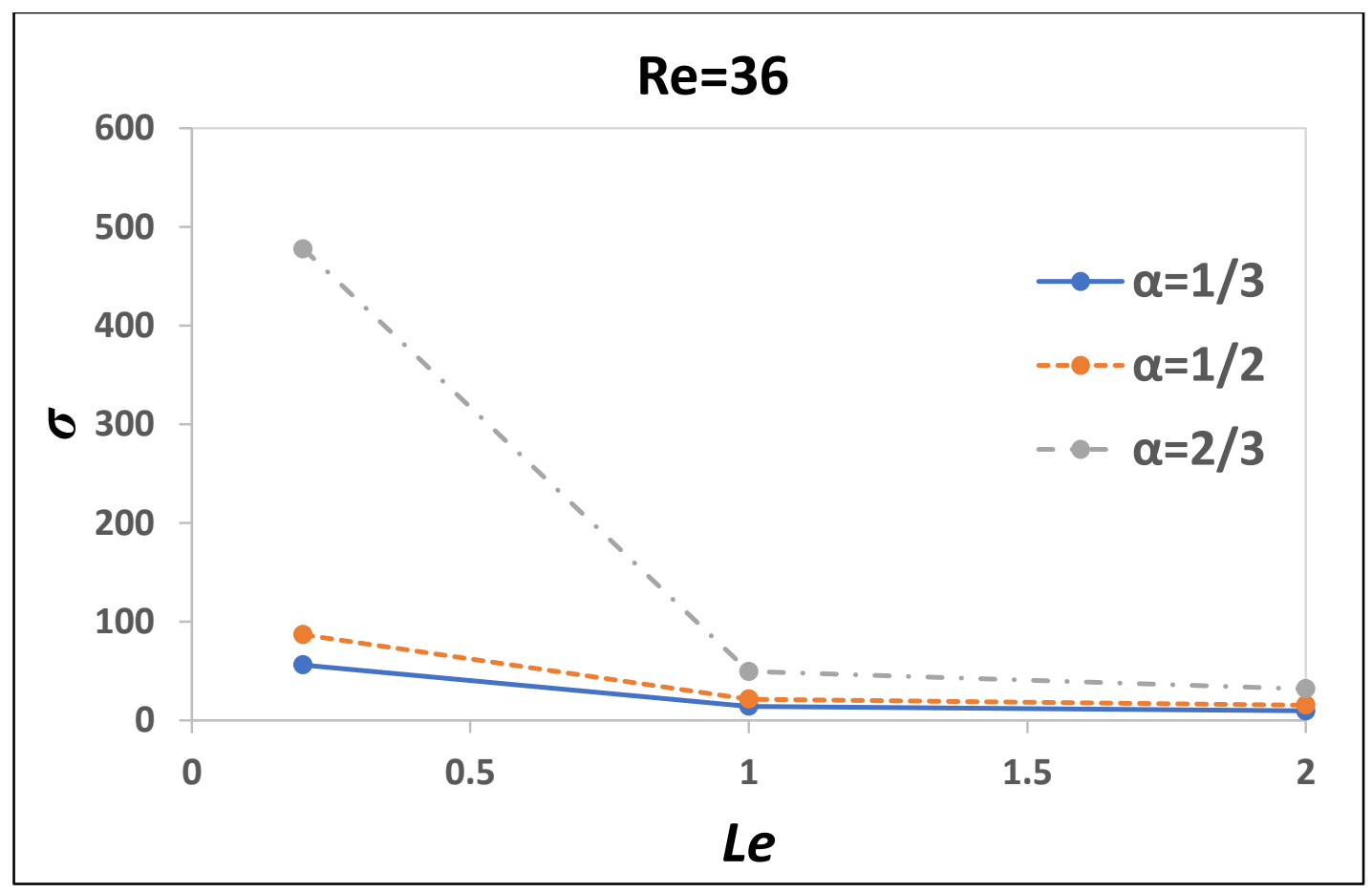

Figure $4.27 b$ - The exponential acceleration rate $v$ s the Lewis number for fixed $R e=36$

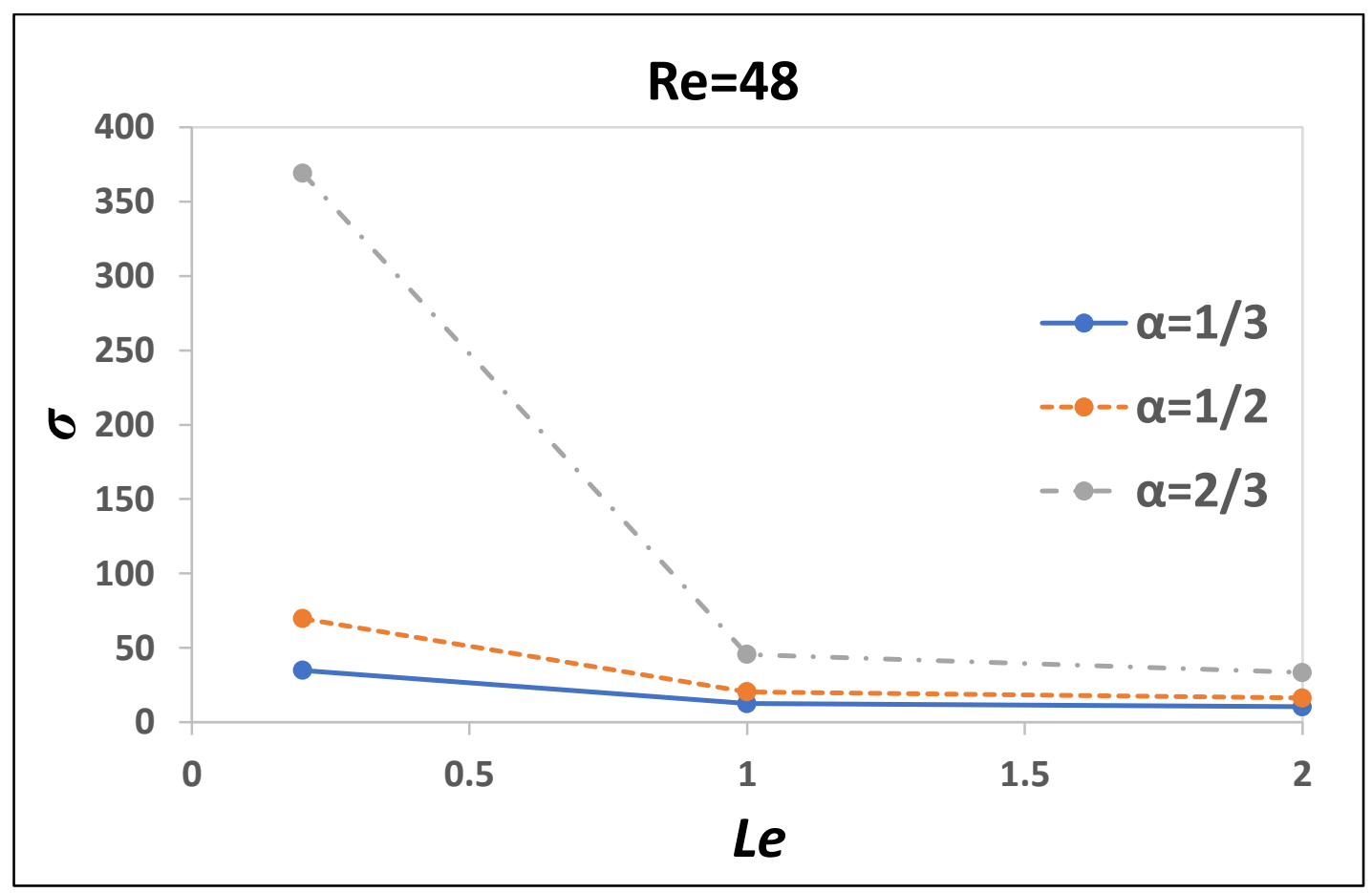

Figure $4.27 c$-The exponential acceleration rate vs the Lewis number for fixed $R e=48$ 


\section{Chapter 5: Conclusions}

Thorough investigation has been performed, computationally, non-equidiffusive FA in obstructed channels and found a profound impact of $L e$ on FA. This effect was compared to that of $\alpha$ and found to be just as strong. The non-equidiffusive scenarios involve non-unity Le, such that in the case of $L e<1$ a promotion of the flame acceleration was discovered. It was also found that Le influences the $\alpha$-dependence. Conversely, a moderation of FA was observed for Le>1 flames. In addition, a unique trend was noticed for the Le impact on Re. Indeed, Le may change the Re-dependence of FA to the opposite trend. These results prove to be instrumental in the understanding of flame moderation techniques in coal mines, or acceleration promotion in novel combustion technologies.

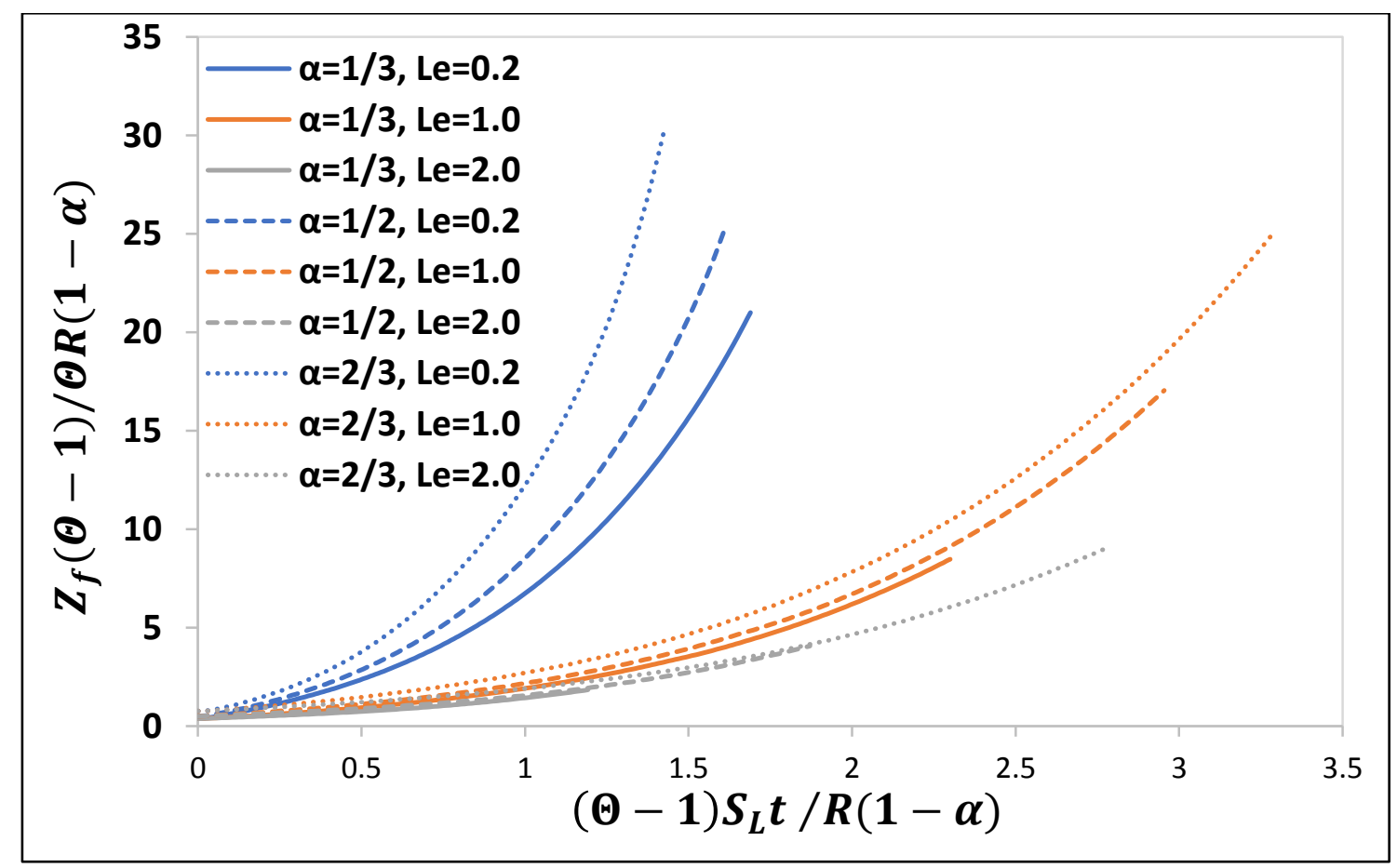

Figure 5.28 - Scaled flame tip position as a function of scaled time, for various $\alpha=1 / 3,1,2,2 / 3$ and Le=0.2, 1.0, 2.0

Figure 5.28 shows the scaled flame tip position versus the scaled time, for the specified range of blockage ratios and Lewis numbers. This plot closely resembles the work of Bychkov et al [7], for an equidiffusive flame analysis. In this figure, each color set is devoted to a separate Lewis number and the different line styles represent various blockage ratios. It can be noticed that for the same Lewis number, the plots tend to collapse into a single curve. This shows a unified role of $L e$ in this flame acceleration mechanism. This was also observed in the Bychkov model, helping to substantiate the accuracy of the results in this present analysis. 


\section{References}

[1] V. Bychkov, A. Petchenko, V. Akkerman, and L.-E. Eriksson, "Theory and modeling of accelerating flames in tubes," Phys. Rev. E - Stat. Nonlinear, Soft Matter Phys., vol. 72, no. 4, 2005.

[2] D.A. Kessler, V.N. Gamezo, and E.S. Oran, "Simulations of flame acceleration and deflagration-to-detonation transitions in methane-air systems," Combust. Flame, vol. 157, no. 11, pp. 2063-2077, 2010.

[3] V. Bychkov, V. Akkerman, G. Fru, A. Petchenko, and L.-E. Eriksson, "Flame acceleration in the early stages of burning in tubes," Combust. Flame, vol. 150, pp. 263-276, 2007.

[4] M. Silvestrini, B. Genova, G. Parisi, and F.J.L. Trujillo, "Flame acceleration and DDT run-up distance for smooth and obstacles filled tubes," J. Loss Prev. Process Ind., vol. 21, no. 5, pp. 555-562, 2008.

[5] G. Ciccarelli, C.J. Fowler, and M. Bardon, "Effect of obstacle size and spacing on the initial stage of flame acceleration in a rough tube," Shock Waves, vol. 14, no. 3, pp. 161166, 2005.

[6] C.T. Johansen and G. Ciccarelli, "Visualization of the unburned gas flow field ahead of an accelerating flame in an obstructed square channel," Combust. Flame, vol. 156, no. 2, pp. 405-416, 2009.

[7] V. Bychkov, D. Valiev, and L.-E. Eriksson, "Physical mechanism of ultrafast flame acceleration," Phys. Rev. Lett., vol. 101, no. 16, pp. 1-4, 2008.

[8] D. Valiev, V. Bychkov, V. Akkerman, C. K. Law, and L.-E. Eriksson, "Flame acceleration in channels with obstacles in the deflagration-to-detonation transition," Combust. Flame, vol. 157, no. 5, pp. 1012-1021, 2010.

[9] O.J. Ugarte, V. Bychkov, J. Sadek, D. Valiev, and V. Akkerman, "Critical role of blockage ratio for flame acceleration in channels with tightly spaced obstacles," Phys. Fluids, vol. 28, no. 9, 093603, 2016. 
[10] J. Yanez J. Kuznetsov M. \& Bykov V. Sudden acceleration of flames in open channels driven by hydraulic resistance, The $24^{\text {th }}$ International Colloquium on the Dynamics of Explosions and Reactive Systems (ICDERS), Taipei, Taiwan, July 28-August 2, 2013, paper \#164, pp. 1-6.

[11] P. Middha, O.R. Hansen, "Predicting deflagration to detonation transition in hydrogen explosions". Process Safety Progress Vol. 27, 192-204 2008.

[12] V. Bychkov, J. Sadek, and V. Akkerman, "Analysis of flame acceleration in open or vented obstructed pipes," Phys. Rev. E, vol. 95, no. 1, pp. 1-9, 2017.

[13] A. Heidari and J. X. Wen, "Numerical simulation of flame acceleration and deflagration to detonation transition in hydrogen-air mixture," Int. J. Hydrogen Energy, vol. 39, no. 36, pp. 21317-21327, 2014. 